\title{
ANALYSIS OF FULLY-MIXED FINITE ELEMENT METHODS FOR THE STOKES-DARCY COUPLED PROBLEM
}

\author{
GABRIEL N. GATICA, RICARDO OYARZÚA, AND FRANCISCO-JAVIER SAYAS
}

\begin{abstract}
In this paper we analyze fully-mixed finite element methods for the coupling of fluid flow with porous media flow in 2D. Flows are governed by the Stokes and Darcy equations, respectively, and the corresponding transmission conditions are given by mass conservation, balance of normal forces, and the Beavers-Joseph-Saffman law. The fully-mixed concept employed here refers to the fact that we consider dual-mixed formulations in both media, which means that the main unknowns are given by the pseudostress and the velocity in the fluid, together with the velocity and the pressure in the porous medium. In addition, the transmission conditions become essential, which leads to the introduction of the traces of the porous media pressure and the fluid velocity as the associated Lagrange multipliers. We apply the Fredholm and BabuškaBrezzi theories to derive sufficient conditions for the unique solvability of the resulting continuous and discrete formulations. In particular, we show that the existence of uniformly bounded discrete liftings of the normal traces simplifies the derivation of the corresponding stability estimates. A feasible choice of subspaces is given by Raviart-Thomas elements of lowest order and piecewise constants for the velocities and pressures, respectively, in both domains, together with continuous piecewise linear elements for the Lagrange multipliers. Finally, several numerical results illustrating the good performance of the method with these discrete spaces, and confirming the theoretical rate of convergence, are provided.
\end{abstract}

\section{INTRODUCTION}

The derivation of suitable numerical methods for the coupling of fluid flow (modelled by the Stokes equations) with porous media flow (modelled by the Darcy equation) has become a very active research area during the last decade (see, e.g. [2, 9, 10, 11, 13, 14, 18], 20, 21], 27, 28, 29, 33, 34, 35, 37, and the references therein). This fact has been motivated by the diverse applications of this coupled model (in petroleum engineering, hydrology, and environmental sciences, to name a few), and also by the increasing need of simpler, more accurate, and more efficient procedures to solve it. Moreover, the latest results available in

Received by the editor June 3, 2009 and, in revised form, July 12, 2010.

2010 Mathematics Subject Classification. Primary 65N15, 65N30, 74F10, 74S05.

The research of the first two authors was partially supported by FONDAP and BASAL projects CMM, Universidad de Chile, by $\mathrm{CI}^{2} \mathrm{MA}$, Universidad de Concepción, and by MECESUP project UCO 0713.

The third author acknowledges support of MEC/FEDER Project MTM2007-63204 and Gobierno de Aragón (Grupo PDIE). This work was developed while the third author was at the University of Minnesota supported by a Spanish MEC grant PR2007-0016. 
the literature also include porous media with cracks, nonlinear problems, and the incorporation of the Brinkman equation in the model (see, e.g. [7, [16, and [38]).

In general, most of the finite element formulations developed for the StokesDarcy coupled problem are based on appropriate combinations of stable elements for the free fluid flow and for the porous medium flow. The first theoretical results in this direction go back to [14] and [28. An iterative subdomain method employing the primal variational formulation and standard finite element subspaces in both domains is proposed in [14. Alternatively, the approach from [28] applies the primal method in the fluid and the dual-mixed one in the porous medium, which means that only the original velocity and pressure unknowns are considered in the Stokes domain, whereas a further unknown (velocity) is added in the Darcy region. The corresponding interface conditions are given by mass conservation, balance of normal forces, and the Beavers-Joseph-Saffman law. Since one of them becomes essential, the trace of the porous medium pressure needs to be incorporated as an additional Lagrange multiplier.

More recently, new mixed finite element discretizations of the variational formulation from [28] have been introduced and analyzed in [20] and 21]. The stability of a specific Galerkin method is the main result in 20. This scheme is defined by using Bernardi-Raugel elements for the velocity in the fluid region, Raviart-Thomas elements of lowest order for the filtration velocity in the porous media, piecewise constants with null mean value for the pressures, and continuous piecewise linear elements for the Lagrange multiplier on the interface. The resulting mixed finite element method is the first one which is conforming for the primal/dual-mixed formulation proposed in 28. The results from 20, are improved in 21, where it is shown that the use of any pair of stable Stokes and Darcy elements implies the stability of the corresponding Stokes-Darcy Galerkin scheme. In particular, this includes Hood-Taylor, Bernardi-Raugel and MINI element for the Stokes region, and Raviart-Thomas of any order for the Darcy domain. The analysis in 21] hinges on the fact that the operator defining the continuous variational formulation is given by a compact perturbation of an invertible mapping.

On the other hand, mortar finite element techniques, discontinuous Galerkin (DG) schemes, and stabilized formulations have also been applied to solve the Stokes-Darcy coupled problem. We first refer to [18 where a nonmatching approach is combined with Hood-Taylor and lowest order Raviart-Thomas spaces in the Stokes and Darcy regions, respectively. Also, stabilized formulations for the free fluid flow combined with stable elements for the Darcy equation are considered in 2 and 34, while stabilized formulations for the porous medium flow combined with stable elements for the Stokes equations are provided in 12 and 37 . Similarly, stabilized formulations in the whole domain are presented in [1] and 33. It is important to notice here that the formulations in [2] and [11] are able to approximate the Stokes and Darcy flows with the same finite element subpaces. Other stabilized formulations with this characteristic are developed in [9], [10, [29, and [35]. In particular, a stabilized piecewise linear/piecewise constant method with an added penalization of pressure jumps over the edges is proposed in [10]. In addition, Crouzeix-Raviart elements for the velocities and piecewise constants for the pressures in both regions, combined with a stabilization term penalizing the jumps of the discontinuous velocities over the edges, are employed in [35]. This approach differs from the one in [9] where the stabilization term depends on the 
normal vectors of the interior edges. In connection with these references we remark that different finite element subspaces in each flow region may lead to different approximation properties for each subproblem. For instance, one could obtain a more accurate velocity field in the Stokes domain than in the Darcy region. On the contrary, employing the same spaces guarantees the same accurateness along the entire domain and leads to simpler and more efficient computational codes.

The purpose of the present work is to contribute in the development of new numerical methods for the 2D Stokes-Darcy coupled problem, allowing on one hand the introduction of further unknowns of physical interest, and on the other hand, the utilization of the same family of finite element subspaces in both media, without requiring any stabilization term. To reach this aim we consider dual-mixed formulations in both domains, which yields the pseudostress and the velocity in the fluid, together with the velocity and the pressure in the porous medium, as the main unknowns. The pressure and the gradient of the velocity in the fluid can then be computed as a very simple postprocess of the above unknowns, in which no numerical differentiation is applied, and hence no further sources of error arise. In addition, since the transmission conditions become essential, we impose them weakly and introduce the traces of the porous media pressure and the fluid velocity, which are also variables of importance from a physical point of view, as the corresponding Lagrange multipliers. Then, we apply the well-known Fredholm and Babuška-Brezzi theories to prove the unique solvability of a suitably chosen continuous formulation and derive sufficient conditions on the finite element subspaces ensuring that the associated Galerkin scheme becomes well posed. In particular, among the several different ways in which the equations and unknowns can be ordered, we choose the one yielding a doubly mixed structure for which the inf-sup conditions of the off-diagonal bilinear forms follow straightforwardly. In this way, the arguments of the continuous analysis can be easily adapted to the discrete case.

The rest of this paper is organized as follows. In Section 2 we introduce the main aspects of the continuous problem, which includes the coupled model, its weak formulation, and the corresponding variational system. The Fredholm theorems and the classical Babuška-Brezzi theory are applied in Section 3 to analyze the continuous problem. Then, in Section 4 we define the Galerkin scheme and derive general hypotheses on the finite element subspaces ensuring that the discrete scheme becomes well posed. In addition, we show that the assumption of existence of uniformly bounded discrete liftings of the normal traces on the interface simplifies the statement of one of the hypotheses. Next, in Section 5 we describe a specific choice of finite element subspaces, namely Raviart-Thomas of lowest order and piecewise constants on both domains, and piecewise linears on the interface, and show that they satisfy all the required assumptions. In particular, we prove that a quasiuniformity condition in a neighborhood of the interface implies the existence of the above-mentioned discrete liftings. Finally, several numerical examples employing these spaces, illustrating the good performance of the method, and confirming the theoretical order of convergence, are reported in Section 6 .

We end this section by summarizing in advance, and according to the already mentioned purpose of the paper, the main advantages of the present fully-mixed approach: it provides either direct finite element approximations or very simple postprocess formulae for several additional quantities of physical interest; it yields, under a special ordering of the resulting equations and unknowns, a unified and 
straightforward analysis of the continuous and discrete formulations; it leads to independent but analogously structured stability assumptions on the finite element subspaces for the Stokes and Darcy regions; and it allows the utilization of the same kind of finite elements in both media, with the consequent simplification of the respective code.

\section{The COnTinuous PROBlem}

2.1. Statement of the model problem. The Stokes-Darcy coupled problem consists of an incompressible viscous fluid occupying a region $\Omega_{\mathrm{S}}$, which flows back and forth across the common interface into a porous medium living in another region $\Omega_{\mathrm{D}}$ and saturated with the same fluid. Physically, we consider a simplified 2D model where $\Omega_{\mathrm{D}}$ is surrounded by a bounded region $\Omega_{\mathrm{S}}$ (see Figure 1 below). Their common interface is supposed to be a Lipschitz curve $\Sigma$ and we assume that $\partial \Omega_{\mathrm{D}}=\Sigma$. The remaining part of the boundary of $\Omega_{\mathrm{S}}$ is also assumed to be a Lipschitz curve $\Gamma_{\mathrm{S}}$. For practical purposes, we can assume that both $\Gamma_{\mathrm{S}}$ and $\Sigma$ are polygons, although this fact will not be used in the general considerations about the formulation of the problem. The unit normal vector field on the boundaries $\mathbf{n}$ is chosen pointing outwards from $\Omega_{\mathrm{S}}$ (and therefore inwards to $\Omega_{\mathrm{D}}$ when seen on $\Sigma$ ). On $\Sigma$ we also consider a unit tangent vector field $\mathbf{t}$ in any fixed orientation of this closed curve.

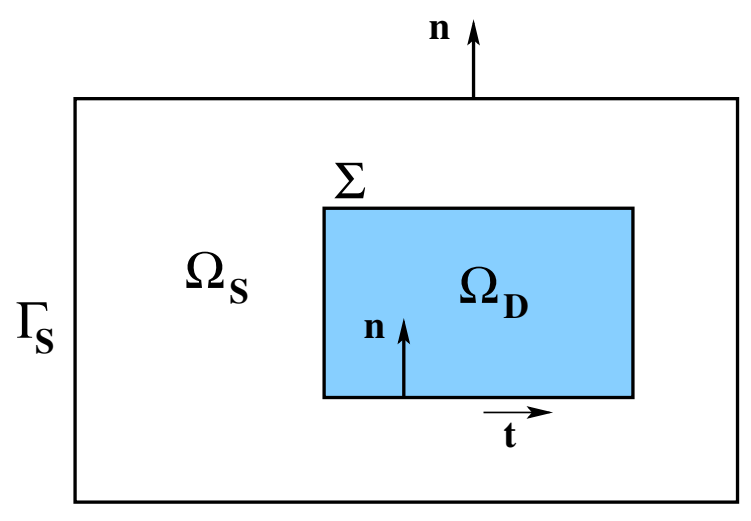

Figure 1. The domains for our simplified 2D Stokes-Darcy model

The mathematical model is defined by two separate groups of equations and a set of coupling terms. In $\Omega_{\mathrm{S}}$, the governing equations are those of the Stokes problem, which are written in the following nonstandard velocity-pressure-pseudostress formulation:

$$
\begin{array}{r}
\boldsymbol{\sigma}_{\mathrm{S}}=-p_{\mathrm{S}} \mathrm{I}+\nu \nabla \mathbf{u}_{\mathrm{S}} \quad \text { in } \quad \Omega_{\mathrm{S}}, \quad \operatorname{div} \boldsymbol{\sigma}_{\mathrm{S}}+\mathbf{f}_{\mathrm{S}}=\mathbf{0} \quad \text { in } \Omega_{\mathrm{S}}, \\
\operatorname{div} \mathbf{u}_{\mathrm{S}}=0 \quad \text { in } \Omega_{\mathrm{S}}, \quad \mathbf{u}_{\mathrm{S}}=\mathbf{0} \quad \text { on } \Gamma_{\mathrm{S}},
\end{array}
$$

where $\nu>0$ is the viscosity of the fluid, $\mathbf{u}_{\mathrm{S}}$ is the fluid velocity, $p_{\mathrm{S}}$ is the pressure, $\boldsymbol{\sigma}_{\mathrm{S}}$ is the pseudostress tensor, I is the $2 \times 2$ identity matrix, and $\mathbf{f}_{\mathrm{S}}$ are known source terms. Here, div is the usual divergence operator acting on vector fields,

$$
\nabla \mathbf{u}=\left(\frac{\partial u_{i}}{\partial x_{j}}\right) \quad \text { and } \quad \operatorname{div} \boldsymbol{\sigma}=\left(\operatorname{div}\left(\sigma_{i 1}, \sigma_{i 2}\right)\right)
$$


i.e., the divergence operator applied to a matrix-valued function (a tensor) is taken row-wise. On the other hand, the flow equations in $\Omega_{\mathrm{D}}$ are those of the linearized Darcy model:

$$
\mathbf{u}_{\mathrm{D}}=-\mathrm{K} \nabla p_{\mathrm{D}} \quad \text { in } \quad \Omega_{\mathrm{D}}, \quad \operatorname{div} \mathbf{u}_{\mathrm{D}}=f_{\mathrm{D}} \quad \text { in } \quad \Omega_{\mathrm{D}},
$$

where the unknowns are the pressure $p_{\mathrm{D}}$ and the flow $\mathbf{u}_{\mathrm{D}}$. The matrix-valued function $\mathrm{K}$, describing permeability of $\Omega_{\mathrm{D}}$ divided by the viscosity $\nu$, satisfies $\mathrm{K}^{t}=$ $\mathrm{K}$, has $L^{\infty}\left(\Omega_{\mathrm{D}}\right)$ components and is uniformly elliptic. Finally, $f_{\mathrm{D}}$ are source terms. We will see that a necessary and sufficient condition for well posedness of the model equations is

$$
\int_{\Omega_{\mathrm{D}}} f_{\mathrm{D}}=0
$$

Finally, the transmission conditions on $\Sigma$ are given by

$$
\begin{aligned}
\mathbf{u}_{\mathrm{S}} \cdot \mathbf{n}=\mathbf{u}_{\mathrm{D}} \cdot \mathbf{n} & \text { on } \quad \Sigma, \\
\boldsymbol{\sigma}_{\mathrm{S}} \mathbf{n}+\nu \kappa^{-1}\left(\mathbf{u}_{\mathrm{S}} \cdot \mathbf{t}\right) \mathbf{t}=-p_{\mathrm{D}} \mathbf{n} & \text { on } \quad \Sigma,
\end{aligned}
$$

where $\kappa:=\frac{\sqrt{(\nu K \mathbf{t}) \cdot \mathbf{t}}}{\alpha}$ is the friction coefficient, and $\alpha$ is a positive parameter to be determined experimentally. The first equation in (2.4) corresponds to mass conservation on $\Sigma$, whereas the second one can be decomposed into its normal and tangential components as follows:

$$
\left(\boldsymbol{\sigma}_{\mathrm{S}} \mathbf{n}\right) \cdot \mathbf{n}=-p_{\mathrm{D}} \quad \text { and } \quad\left(\boldsymbol{\sigma}_{\mathrm{S}} \mathbf{n}\right) \cdot \mathbf{t}=-\nu \kappa^{-1}\left(\mathbf{u}_{\mathrm{S}} \cdot \mathbf{t}\right) \quad \text { on } \quad \Sigma,
$$

which constitute the balance of normal forces and the Beavers-Joseph-Saffman law, respectively. The latter establishes that the slip velocity along $\Sigma$ is proportional to the shear stress along $\Sigma$ (assuming also, based on experimental evidences, that $\mathbf{u}_{\mathrm{D}} \cdot \mathbf{t}$ is negligible). We refer to [6], [26, and [36] for further details on this interface condition. Throughout the rest of the paper we assume, without loss of generality, that $\kappa$ is a positive constant.

The description of our model problem is completed by observing that the equations in the Stokes domain (cf. (2.1)) can be rewritten equivalently as

$$
\begin{aligned}
\nu^{-1} \boldsymbol{\sigma}_{\mathrm{S}}^{d} & =\nabla \mathbf{u}_{\mathrm{S}} \quad \text { in } \quad \Omega_{\mathrm{S}}, \quad \operatorname{div} \boldsymbol{\sigma}_{\mathrm{S}}+\mathbf{f}_{\mathrm{S}}=\mathbf{0} \quad \text { in } \Omega_{\mathrm{S}}, \\
p_{\mathrm{S}} & =-\frac{1}{2} \operatorname{tr} \boldsymbol{\sigma}_{\mathrm{S}} \quad \text { in } \quad \Omega_{\mathrm{S}}, \quad \mathbf{u}_{\mathrm{S}}=\mathbf{0} \quad \text { on } \quad \Gamma_{\mathrm{S}},
\end{aligned}
$$

where tr stands for the usual trace of tensors, that is, $\operatorname{tr} \tau:=\tau_{11}+\tau_{22}$, and

$$
\boldsymbol{\tau}^{d}:=\boldsymbol{\tau}-\frac{1}{2}(\operatorname{tr} \boldsymbol{\tau}) \mathrm{I}
$$

is the deviatoric part of the tensor $\tau$. The third equation in (2.5) allows us to eliminate $p_{\mathrm{S}}$ from the system and compute it as a simple postprocess of the solution. Similarly, the first equation in (2.5) yields a straightforward postprocess formula for the gradient of the velocity in the fluid. Note that a constant $c$ added to both $p_{\mathrm{S}}$ and $p_{\mathrm{D}}$ is not perceived by the system; its only effect is a correction in $\boldsymbol{\sigma}_{\mathrm{S}}$ that has to be subtracted $c$ times the identity matrix.

We end this section by remarking that, though the geometry described by Figure 11 was chosen to simplify the presentation, the case of a fluid flowing only across a part of the boundary of the porous medium does not really yield further complications for the analysis in the present paper. For instance, if we consider a fluid over the porous medium, $\partial \Omega_{\mathrm{S}}$ stays given by $\Gamma_{\mathrm{S}} \cup \Sigma$, but now with both curves meeting 
at their end points, whereas a new piece of $\partial \Omega_{\mathrm{D}}$, say $\Gamma$, such that $\partial \Omega_{\mathrm{D}}=\Sigma \cup \Gamma$, needs to be identified. In this case, besides the equations given in the present section (which hold now with the notations introduced here), a boundary condition on $\Gamma$ needs to be added. Following [18] and [28] (see also [16]), one usually considers the homogeneous Neumann condition:

$$
\mathbf{u}_{\mathrm{D}} \cdot \mathbf{n}=0 \text { on } \Gamma,
$$

which constitutes a no flow assumption through $\Gamma$. We refer to [18] for further details and emphasize that only minor modifications will need to be incorporated into the forthcoming analysis. In particular, this is certainly valid for the discrete analysis, which is illustrated by two numerical examples reported below in Section 6. Alternatively, instead of (2.6) one can consider the homogeneous Dirichlet condition:

$$
p_{\mathrm{D}}=0 \text { on } \Gamma \text {, }
$$

which, as will be explained at the end of Section 3 below, becomes a unique solvability condition for the resulting variational formulation.

2.2. The weak formulation. Let us first introduce some general functional spaces. If $\mathcal{O}$ is a domain, $\Gamma$ is a closed Lipschitz curve, and $r \in \mathbb{R}$, we define

$$
\mathbf{H}^{r}(\mathcal{O}):=\left[H^{r}(\mathcal{O})\right]^{2}, \quad \mathbb{H}^{r}(\mathcal{O}):=\left[H^{r}(\mathcal{O})\right]^{2 \times 2}, \quad \text { and } \quad \mathbf{H}^{r}(\Gamma):=\left[H^{r}(\Gamma)\right]^{2} .
$$

In the particular case $r=0$ we usually write $\mathbf{L}^{2}(\mathcal{O}), \mathbb{L}^{2}(\mathcal{O})$, and $\mathbf{L}^{2}(\Gamma)$ instead of $\mathbf{H}^{0}(\mathcal{O}), \mathbb{H}^{0}(\mathcal{O})$, and $\mathbf{H}^{0}(\Gamma)$, respectively. The corresponding norms are denoted by $\|\cdot\|_{r, \mathcal{O}}\left(\right.$ for $H^{r}(\mathcal{O}), \mathbf{H}^{r}(\mathcal{O})$, and $\left.\mathbb{H}^{r}(\mathcal{O})\right)$ and $\|\cdot\|_{r, \Gamma}\left(\right.$ for $H^{r}(\Gamma)$ and $\left.\mathbf{H}^{r}(\Gamma)\right)$.

Also, the Hilbert space

$$
\mathbf{H}(\operatorname{div} ; \mathcal{O}):=\left\{\mathbf{w} \in \mathbf{L}^{2}(\mathcal{O}): \quad \operatorname{div} \mathbf{w} \in L^{2}(\mathcal{O})\right\}
$$

is standard in the realm of mixed problems (see [8] or [23] for instance). The space of matrix-valued functions whose rows belong to $\mathbf{H}(\operatorname{div} ; \mathcal{O})$ will be denoted $\mathbb{H}(\operatorname{div} ; \mathcal{O})$. The Hilbert norms of $\mathbf{H}(\operatorname{div} ; \mathcal{O})$ and $\mathbb{H}(\mathbf{d i v} ; \mathcal{O})$ are denoted by $\|\cdot\|_{\text {div; } \mathcal{O}}$ and $\|\cdot\|_{\operatorname{div} ; \mathcal{O}}$, respectively. Note that if $\boldsymbol{\tau} \in \mathbb{H}(\operatorname{div} ; \mathcal{O})$, then $\operatorname{div} \boldsymbol{\tau} \in \mathbf{L}^{2}(\mathcal{O})$. Note also that $\mathbb{H}(\mathbf{d i v} ; \mathcal{O})$ can be characterized as the space of matrix-valued functions $\boldsymbol{\tau}$ such that $\mathbf{c}^{t} \boldsymbol{\tau} \in \mathbf{H}(\operatorname{div} ; \mathcal{O})$ for any constant column vector $\mathbf{c}$. In addition, it is easy to see that there holds:

$$
\mathbb{H}(\operatorname{div} ; \mathcal{O})=\mathbb{H}_{0}(\operatorname{div} ; \mathcal{O}) \oplus \mathbb{P}_{0}(\mathcal{O}) \mathrm{I},
$$

where

$$
\mathbb{H}_{0}(\operatorname{div} ; \mathcal{O}):=\left\{\boldsymbol{\sigma} \in \mathbb{H}(\operatorname{div} ; \mathcal{O}): \quad \int_{\mathcal{O}} \operatorname{tr} \boldsymbol{\sigma}=0\right\}
$$

and $\mathbb{P}_{0}(\mathcal{O})$ is the space of constant polynomials on $\mathcal{O}$. More precisely, each $\tau \in$ $\mathbb{H}(\mathbf{d i v} ; \mathcal{O})$ can be decomposed uniquely as:

$$
\boldsymbol{\tau}=\boldsymbol{\tau}_{0}+c \mathrm{I}, \quad \text { with } \quad \boldsymbol{\tau}_{0} \in \mathbb{H}_{0}(\operatorname{div} ; \mathcal{O}) \quad \text { and } \quad c:=\frac{1}{2|\mathcal{O}|} \int_{\mathcal{O}} \operatorname{tr} \boldsymbol{\tau} \in \mathbb{R}
$$

This decomposition will be utilized below to analyze the weak formulation of our problem.

On the other hand, for simplicity of notation we will also denote, with $\star \in\{\mathrm{S}, \mathrm{D}\}$

$$
(u, v)_{\star}:=\int_{\Omega_{\star}} u v, \quad(\mathbf{u}, \mathbf{v})_{\star}:=\int_{\Omega_{\star}} \mathbf{u} \cdot \mathbf{v}, \quad(\boldsymbol{\sigma}, \boldsymbol{\tau})_{\star}:=\int_{\Omega_{\star}} \boldsymbol{\sigma}: \boldsymbol{\tau},
$$


where $\boldsymbol{\sigma}: \boldsymbol{\tau}=\operatorname{tr}\left(\boldsymbol{\sigma}^{t} \boldsymbol{\tau}\right)=\sum_{i j=1}^{2} \sigma_{i j} \tau_{i j}$. Note the following simple and useful identity

$$
\boldsymbol{\sigma}^{d}: \boldsymbol{\tau}^{d}=\boldsymbol{\sigma}^{d}: \boldsymbol{\tau}=\boldsymbol{\sigma}: \boldsymbol{\tau}-\frac{1}{2}(\operatorname{tr} \boldsymbol{\sigma})(\operatorname{tr} \boldsymbol{\tau})
$$

The symbols for the $L^{2}(\Sigma)$ and $\mathbf{L}^{2}(\Sigma)$ inner products

$$
\langle\xi, \lambda\rangle_{\Sigma}:=\int_{\Sigma} \xi \lambda, \quad\langle\boldsymbol{\xi}, \boldsymbol{\lambda}\rangle_{\Sigma}:=\int_{\Sigma} \boldsymbol{\xi} \cdot \boldsymbol{\lambda}
$$

will also be employed for their extensions as the duality products $H^{-1 / 2}(\Sigma) \times$ $H^{1 / 2}(\Sigma)$ and $\mathbf{H}^{-1 / 2}(\Sigma) \times \mathbf{H}^{1 / 2}(\Sigma)$, respectively.

The unknowns in the weak (mixed) formulation will be the two unknowns in (2.2) and the unknowns of (2.5) without the pressure $p_{\mathrm{S}}$. The corresponding spaces will be:

$$
\boldsymbol{\sigma}_{\mathrm{S}} \in \mathbb{H}\left(\operatorname{div} ; \Omega_{\mathrm{S}}\right), \quad \mathbf{u}_{\mathrm{S}} \in \mathbf{L}^{2}\left(\Omega_{\mathrm{S}}\right), \quad \mathbf{u}_{\mathrm{D}} \in \mathbf{H}\left(\operatorname{div} ; \Omega_{\mathrm{D}}\right), \quad p_{\mathrm{D}} \in L^{2}\left(\Omega_{\mathrm{D}}\right) .
$$

In addition, we will need to define two unknowns on the coupling boundary

$$
\boldsymbol{\varphi}:=-\mathbf{u}_{\mathrm{S}} \in \mathbf{H}^{1 / 2}(\Sigma), \quad \lambda:=p_{\mathrm{D}} \in H^{1 / 2}(\Sigma) .
$$

Note that in principle the spaces for $\mathbf{u}_{\mathrm{S}}$ and $p_{\mathrm{D}}$ do not allow enough regularity for the traces above to exist. However, solutions of (2.2) and (2.5) have these unknowns in $\mathbf{H}^{1}\left(\Omega_{\mathrm{S}}\right)$ and $H^{1}\left(\Omega_{\mathrm{D}}\right)$, respectively.

In order to obtain the weak formulation of (2.2)-2.4 -2.5), we apply the divergence theorem to the first equation in both (2.2) and (2.5), that is to those equations relating $\boldsymbol{\sigma}_{\mathrm{S}}$ and $\mathbf{u}_{\mathrm{D}}$ to other magnitudes. Then, due to the mixed nature of the model, the Dirichlet condition in (2.5) and the traces of $p_{\mathrm{D}}$ and $\mathbf{u}_{\mathrm{S}}$ on $\Sigma$ become natural and hence they are incorporated directly in the weak formulation. On the contrary, both transmission conditions in (2.4) become essential, whence they have to be imposed independently, thus yielding the introduction of the auxiliary unknowns (2.12) as the corresponding Lagrange multipliers. According to the above, the weak equations can be written as follows: we look for the unknowns

$$
\begin{aligned}
\left(\boldsymbol{\sigma}_{\mathrm{S}}, \mathbf{u}_{\mathrm{S}}, \boldsymbol{\varphi}\right) & \in \mathbb{H}\left(\operatorname{div} ; \Omega_{\mathrm{S}}\right) \times \mathbf{L}^{2}\left(\Omega_{\mathrm{S}}\right) \times \mathbf{H}^{1 / 2}(\Sigma) \\
\left(\mathbf{u}_{\mathrm{D}}, p_{\mathrm{D}}, \lambda\right) & \in \mathbf{H}\left(\operatorname{div} ; \Omega_{\mathrm{D}}\right) \times L^{2}\left(\Omega_{\mathrm{D}}\right) \times H^{1 / 2}(\Sigma)
\end{aligned}
$$

satisfying two variational equations

$$
\begin{aligned}
& \nu^{-1}\left(\boldsymbol{\sigma}_{\mathrm{S}}^{d}, \boldsymbol{\tau}_{\mathrm{S}}^{d}\right)_{\mathrm{S}}+\left(\operatorname{div} \boldsymbol{\tau}_{\mathrm{S}}, \mathbf{u}_{\mathrm{S}}\right)_{\mathrm{S}}+\left\langle\boldsymbol{\tau}_{\mathrm{S}} \mathbf{n}, \boldsymbol{\varphi}\right\rangle_{\Sigma}=0 \quad \forall \boldsymbol{\tau}_{\mathrm{S}} \in \mathbb{H}\left(\operatorname{div} ; \Omega_{\mathrm{S}}\right), \\
& \left(\mathrm{K}^{-1} \mathbf{u}_{\mathrm{D}}, \mathbf{v}_{\mathrm{D}}\right)_{\mathrm{D}}-\left(\operatorname{div} \mathbf{v}_{\mathrm{D}}, p_{\mathrm{D}}\right)_{\mathrm{D}}-\left\langle\mathbf{v}_{\mathrm{D}} \cdot \mathbf{n}, \lambda\right\rangle_{\Sigma}=0 \\
& \forall \mathbf{v}_{\mathrm{D}} \in \mathbf{H}\left(\mathbf{d i v} ; \Omega_{\mathrm{D}}\right),
\end{aligned}
$$

two differential equations

$$
\begin{aligned}
& \operatorname{div} \sigma_{\mathrm{S}}+\mathbf{f}_{\mathrm{S}}=\mathbf{0} \quad \text { in } \Omega_{\mathrm{S}}, \\
& \operatorname{div} \mathbf{u}_{\mathrm{D}}=f_{\mathrm{D}} \quad \text { in } \quad \Omega_{\mathrm{D}},
\end{aligned}
$$


with source terms $\mathbf{f}_{\mathrm{S}} \in \mathbf{L}^{2}\left(\Omega_{\mathrm{S}}\right)$ and $f_{\mathrm{D}} \in L^{2}\left(\Omega_{\mathrm{D}}\right)$, and two restrictions on the boundary

$$
\begin{aligned}
& \boldsymbol{\varphi} \cdot \mathbf{n}+\mathbf{u}_{\mathrm{D}} \cdot \mathbf{n}=0 \text { in } \quad H^{-1 / 2}(\Sigma), \\
& \boldsymbol{\sigma}_{\mathrm{S}} \mathbf{n}+\lambda \mathbf{n}-\nu \kappa^{-1}(\boldsymbol{\varphi} \cdot \mathbf{t}) \mathbf{t}=0 \quad \text { in } \quad \mathbf{H}^{-1 / 2}(\Sigma) .
\end{aligned}
$$

The apparently wrong sign in the term where $\lambda$ appears in the second equation of (2.17) is due to the fact that the normal on $\Sigma$ points inwards from the point of view of $\Omega_{\mathrm{D}}$.

Different orderings of the equations and unknowns will emphasize different structural properties of the system. We will show three possibilities shortly.

Theorem 2.1. Assume that we have a solution (2.13) of the system (2.14) $-(2.15)$ (2.16)-(2.17) and that we define $p_{\mathrm{S}}:=-\frac{1}{2} \operatorname{tr} \boldsymbol{\sigma}_{\mathrm{S}}$. Then $\mathbf{u}_{\mathrm{S}} \in \mathbf{H}^{1}\left(\Omega_{\mathrm{S}}\right), p_{\mathrm{D}} \in$ $H^{1}\left(\Omega_{\mathrm{D}}\right), \boldsymbol{\varphi}=-\mathbf{u}_{\mathrm{S}}$ on $\Sigma, \lambda=p_{\mathrm{D}}$ on $\Sigma$ and we have a solution of the system (2.1) $-(2.2)-(2.4)$.

Proof. It is a simple application of well-known results on distribution theory and Sobolev spaces of $H^{1}(\mathcal{O})$ and $\mathbf{H}(\operatorname{div} ; \mathcal{O})$ type.

2.3. The variational system. The weak system (2.14)-(2.15)-(2.16)-(2.17) can be described in purely variational form. To do that, we now test the equations (2.16) and the first equation of (2.17) with arbitrary $\mathbf{v} \in \mathbf{L}^{2}\left(\Omega_{\mathrm{S}}\right), q \in L^{2}\left(\Omega_{\mathrm{D}}\right)$, and $\xi \in H^{1 / 2}(\Sigma)$, respectively, which give

$$
\begin{array}{cc}
\left(\operatorname{div} \boldsymbol{\sigma}_{\mathrm{S}}, \mathbf{v}_{\mathrm{S}}\right)_{\mathrm{S}}=-\left(\mathbf{f}_{\mathrm{S}}, \mathbf{v}_{\mathrm{S}}\right)_{\mathrm{S}} & \forall \mathbf{v}_{\mathrm{S}} \in \mathbf{L}^{2}\left(\Omega_{\mathrm{S}}\right), \\
\left(\operatorname{div} \mathbf{u}_{\mathrm{D}}, q_{\mathrm{D}}\right)_{\mathrm{D}}=\left(f_{\mathrm{D}}, q_{\mathrm{D}}\right)_{\mathrm{D}} & \forall q_{\mathrm{D}} \in L^{2}\left(\Omega_{\mathrm{D}}\right),
\end{array}
$$

and

$$
\langle\boldsymbol{\varphi} \cdot \mathbf{n}, \xi\rangle_{\Sigma}+\left\langle\mathbf{u}_{\mathrm{D}} \cdot \mathbf{n}, \xi\right\rangle_{\Sigma}=0 \quad \forall \xi \in H^{1 / 2}(\Sigma) .
$$

In addition, for convenience of the subsequent analysis we consider the decomposition (2.8)-(2.9) with $\mathcal{O}=\Omega_{\mathrm{S}}$, and from now on redefine the fluid pseudostress as

$$
\sigma_{\mathrm{S}}+\mu \mathrm{I} \quad \text { with the new unknowns } \quad \sigma_{\mathrm{S}} \in \mathbb{H}_{0}\left(\operatorname{div} ; \Omega_{\mathrm{S}}\right) \quad \text { and } \quad \mu \in \mathbb{R} .
$$

In this way, the variational formulation of the second transmission condition in (2.17) becomes

$$
\begin{array}{r}
\left\langle\boldsymbol{\sigma}_{\mathrm{S}} \mathbf{n}, \boldsymbol{\psi}\right\rangle_{\Sigma}+\langle\boldsymbol{\psi} \cdot \mathbf{n}, \lambda\rangle_{\Sigma}-\nu \kappa^{-1}\langle\boldsymbol{\varphi} \cdot \mathbf{t}, \boldsymbol{\psi} \cdot \mathbf{t}\rangle_{\Sigma}+\mu\langle\boldsymbol{\psi} \cdot \mathbf{n}, 1\rangle_{\Sigma}=0 \\
\forall \boldsymbol{\psi} \in \mathbf{H}^{1 / 2}(\Sigma),
\end{array}
$$

and the equation (2.14) is rewritten, equivalently, as

$$
\nu^{-1}\left(\boldsymbol{\sigma}_{\mathrm{S}}^{d}, \boldsymbol{\tau}_{\mathrm{S}}^{d}\right)_{\mathrm{S}}+\left(\operatorname{div} \boldsymbol{\tau}_{\mathrm{S}}, \mathbf{u}_{\mathrm{S}}\right)_{\mathrm{S}}+\left\langle\boldsymbol{\tau}_{\mathrm{S}} \mathbf{n}, \boldsymbol{\varphi}\right\rangle_{\Sigma}=0 \quad \forall \boldsymbol{\tau}_{\mathrm{S}} \in \mathbb{H}_{0}\left(\operatorname{div} ; \Omega_{\mathrm{S}}\right)
$$

and

$$
\eta\langle\boldsymbol{\varphi} \cdot \mathbf{n}, 1\rangle_{\Sigma}=0 \quad \forall \eta \in \mathbb{R} .
$$

As a consequence of the above, we find that the resulting variational formulation reduces to a system of seven equations $((2.15),(2.18)-(2.20),(2.22)-(2.24))$ and 
seven unknowns, which can be written in terms of the following nine bilinear forms:

$$
\begin{array}{llllll}
\mathrm{A}: & \nu^{-1}\left(\boldsymbol{\sigma}_{\mathrm{S}}^{d}, \boldsymbol{\tau}_{\mathrm{S}}^{d}\right)_{\mathrm{S}} & \mathrm{D}: & \nu \kappa^{-1}\langle\boldsymbol{\varphi} \cdot \mathbf{t}, \boldsymbol{\psi} \cdot \mathbf{t}\rangle_{\Sigma} & \mathrm{G}: & -\left(\operatorname{div} \mathbf{u}_{\mathrm{D}}, q_{\mathrm{D}}\right)_{\mathrm{D}}, \\
\mathrm{B}: & \left(\boldsymbol{d i v} \boldsymbol{\sigma}_{\mathrm{S}}, \mathbf{v}_{\mathrm{S}}\right)_{\mathrm{S}} & \mathrm{E}: & \langle\boldsymbol{\varphi} \cdot \mathbf{n}, \xi\rangle_{\Sigma} & \mathrm{H}: & -\left\langle\mathbf{u}_{\mathrm{D}} \cdot \mathbf{n}, \xi\right\rangle_{\Sigma}, \\
\mathrm{C}: & \left\langle\boldsymbol{\sigma}_{\mathrm{S}} \mathbf{n}, \boldsymbol{\psi}\right\rangle_{\Sigma} & \mathrm{F}: & \left(\mathrm{K}^{-1} \mathbf{u}_{\mathrm{D}}, \mathbf{v}_{\mathrm{D}}\right)_{\mathrm{D}} & \mathrm{J}: & \eta\langle\boldsymbol{\varphi} \cdot \mathbf{n}, 1\rangle_{\Sigma} .
\end{array}
$$

On the left of each column of (2.25) we have added a key letter for the nine different bilinear forms (or related operators). It is easy to see that all of these bilinear forms are bounded. Also, those with both arguments in the same space

$$
\mathrm{A}: \quad \nu^{-1}\left(\boldsymbol{\sigma}_{\mathrm{S}}^{d}, \boldsymbol{\tau}_{\mathrm{S}}^{d}\right)_{\mathrm{S}}, \quad \mathrm{D}: \quad \nu \kappa^{-1}\langle\boldsymbol{\varphi} \cdot \mathbf{t}, \boldsymbol{\psi} \cdot \mathbf{t}\rangle_{\Sigma}, \quad \mathrm{F}: \quad\left(\mathrm{K}^{-1} \mathbf{u}_{\mathrm{D}}, \mathbf{v}_{\mathrm{D}}\right)_{\mathrm{D}}
$$

are symmetric and positive semidefinite. In addition, the bilinear forms

$$
\mathrm{D}: \quad \nu \kappa^{-1}\langle\boldsymbol{\varphi} \cdot \mathbf{t}, \boldsymbol{\psi} \cdot \mathbf{t}\rangle_{\Sigma}, \quad \mathrm{E}:\langle\boldsymbol{\varphi} \cdot \mathbf{n}, \xi\rangle_{\Sigma}
$$

are compact by the compact inclusion of $\mathbf{H}^{1 / 2}(\Sigma)$ in $\mathbf{L}^{2}(\Sigma)$.

Now, it is quite clear that there are many different ways of ordering the variational system. In order to illustrate this fact and identify a suitable form, in Table 1 below we show three options, emphasizing different structural properties of them. On the left of each row we indicate the corresponding equation. Besides the row and the column involving the unknown $\mu$, we observe in ((1)) that the remaining equations show two blocks on the diagonal: the Stokes block in mixed form with a penalization term and the Darcy block in mixed form. The coupling is limited to $\mathrm{E}$ and $\mathrm{E}^{t}$. Changing the sign of the fourth equation we obtain a symmetric system, whereas changing the sign of the second and third equations we see the sign of the underlying quadratic form: off-diagonal terms compose a skew-symmetric matrix and diagonal terms are positive semidefinite. Similarly, beside again the row and the column involving $\mu$, we observe in ((2)) that the variables are grouped by character and a different mixed structure, with a nonsymmetric and negative semidefinite penalization term, is recovered. Nevertheless, a good feature of this system is the fact that $\mathrm{D}$ and $\mathrm{E}$ are compact, so taking away the penalization term, the remaining system consists of a purely mixed problem, which can be decoupled in two mixed problems. On the other hand, ((3)) shows a particular overlapping of the Stokes and Darcy blocks, which, at first, seems to mix everything in an inconvenient way. However, a closer look to this ordering allows us to identify a doubly-mixed structure in which the interior mixed formulation contains the same penalization term observed in ((2)). Moreover, all the block bilinear forms, except the one defining the penalization term, show a diagonal structure, which constitutes an advantageous feature when proving the corresponding inf-sup conditions.

Throughout the rest of the paper we adopt the structure ((3)) for our analysis. This means that we group unknowns and spaces as follows:

$(2.26)$

$$
\begin{gathered}
\underline{\boldsymbol{\sigma}}:=\left(\boldsymbol{\sigma}_{\mathrm{S}}, \mathbf{u}_{\mathrm{D}}, \boldsymbol{\varphi}, \lambda\right) \in \mathbb{X}_{0}:=\mathbb{H}_{0}\left(\operatorname{div} ; \Omega_{\mathrm{S}}\right) \times \mathbf{H}\left(\operatorname{div} ; \Omega_{\mathrm{D}}\right) \times \mathbf{H}^{1 / 2}(\Sigma) \times H^{1 / 2}(\Sigma), \\
\underline{\mathbf{u}}:=\left(\mathbf{u}_{\mathrm{S}}, p_{\mathrm{D}}, \mu\right) \in \mathbb{M}:=\mathbf{L}^{2}\left(\Omega_{\mathrm{S}}\right) \times L^{2}\left(\Omega_{\mathrm{D}}\right) \times \mathbb{R} .
\end{gathered}
$$

In this way, the variational system of our problem reads: Find $(\underline{\boldsymbol{\sigma}}, \underline{\mathbf{u}}) \in \mathbb{X}_{0} \times \mathbb{M}$ such that

$$
\begin{array}{rlrl}
\mathcal{A}(\underline{\boldsymbol{\sigma}}, \underline{\boldsymbol{\tau}})+\mathcal{B}(\underline{\boldsymbol{\tau}}, \underline{\mathbf{u}}) & =\mathcal{F}(\underline{\boldsymbol{\tau}}) & \forall \underline{\boldsymbol{\tau}}:=\left(\boldsymbol{\tau}_{\mathrm{S}}, \mathbf{v}_{\mathrm{D}}, \boldsymbol{\psi}, \xi\right) \in \mathbb{X}_{0} \\
\mathcal{B}(\underline{\boldsymbol{\sigma}}, \underline{\mathbf{v}}) & =\mathcal{G}(\underline{\mathbf{v}}) \quad \forall \underline{\mathbf{v}}:=\left(\mathbf{v}_{\mathrm{S}}, q_{\mathrm{D}}, \eta\right) \in \mathbb{M}
\end{array}
$$


TABLE 1. Three different forms of structuring the variational system.

\begin{tabular}{|c|ccc|ccc||c|}
\hline$((1))$ & $\boldsymbol{\sigma}_{\mathrm{S}}$ & $\mathbf{u}_{\mathrm{S}}$ & $\boldsymbol{\varphi}$ & $\mathbf{u}_{\mathrm{D}}$ & $p_{\mathrm{D}}$ & $\lambda$ & $\mu$ \\
\hline$(2.23)$ & $\mathrm{A}$ & $\mathrm{B}^{t}$ & $\mathrm{C}^{t}$ & & & & \\
$(\underline{(2.18)}$ & $\mathrm{B}$ & & & & & & \\
$(2.22)$ & $\mathrm{C}$ & & $-\mathrm{D}$ & & & $\mathrm{E}^{t}$ & $\mathrm{~J}^{t}$ \\
\hline$(2.15)$ & & & & $\mathrm{F}$ & $\mathrm{G}^{t}$ & $\mathrm{H}^{t}$ & \\
$(\underline{(2.19)}$ & & & & $-\mathrm{G}$ & & & \\
$(2.20)$ & & & $\mathrm{E}$ & $-\mathrm{H}$ & & & \\
\hline \hline$(2.24)$ & & & $\mathrm{J}$ & & & \\
\hline
\end{tabular}

\begin{tabular}{|c|cc|cccc||c|}
\hline$((2))$ & $\boldsymbol{\sigma}_{\mathrm{S}}$ & $\mathbf{u}_{\mathrm{D}}$ & $\mathbf{u}_{\mathrm{S}}$ & $p_{\mathrm{D}}$ & $\boldsymbol{\varphi}$ & $\lambda$ & $\mu$ \\
\hline$(2.23)$ & $\mathrm{A}$ & & $\mathrm{B}^{t}$ & & $\mathrm{C}^{t}$ & & \\
$(2.15)$ & & $\mathrm{F}$ & & $\mathrm{G}^{t}$ & & $\mathrm{H}^{t}$ & \\
\hline$(2.18)$ & $\mathrm{B}$ & & & & & & \\
$(\underline{(2.19)}$ & & $\mathrm{G}$ & & & & & \\
$(\underbrace{(2.20)}_{(2.22)}$ & $\mathrm{C}$ & & & & $-\mathrm{D}$ & $\mathrm{E}^{t}$ & $\mathrm{~J}^{t}$ \\
\hline \hline$(2.24)$ & & $\mathrm{H}$ & & & $-\mathrm{E}$ & & \\
\hline
\end{tabular}

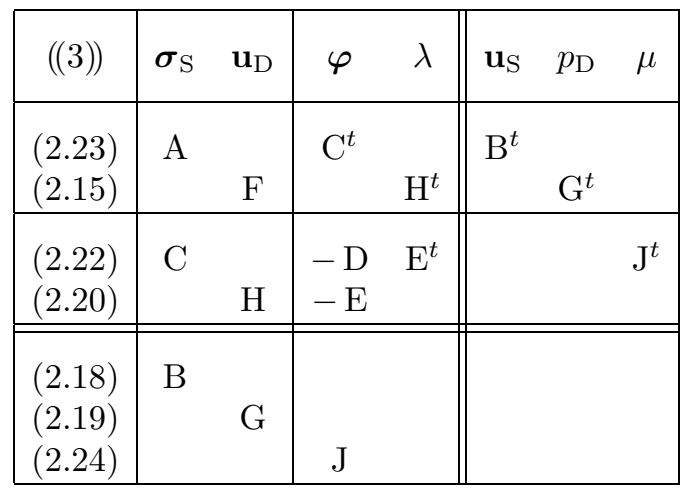

where

$$
\mathcal{F}(\underline{\boldsymbol{\tau}}):=0, \quad \mathcal{G}(\underline{\mathbf{v}})=\mathcal{G}\left(\left(\mathbf{v}_{\mathrm{S}}, q_{\mathrm{D}}, \eta\right)\right):=-\left(\mathbf{f}_{\mathrm{S}}, \mathbf{v}_{\mathrm{S}}\right)_{\mathrm{S}}-\left(f_{\mathrm{D}}, q_{\mathrm{D}}\right),
$$

and $\mathcal{A}$ and $\mathcal{B}$ are the bounded bilinear forms defined by

$$
\begin{gathered}
\mathcal{A}(\underline{\boldsymbol{\sigma}}, \underline{\boldsymbol{\tau}})=\mathbf{a}\left(\left(\boldsymbol{\sigma}_{\mathrm{S}}, \mathbf{u}_{\mathrm{D}}\right),\left(\boldsymbol{\tau}_{\mathrm{S}}, \mathbf{v}_{\mathrm{D}}\right)\right)+\mathbf{b}\left(\left(\boldsymbol{\tau}_{\mathrm{S}}, \mathbf{v}_{\mathrm{D}}\right),(\boldsymbol{\varphi}, \lambda)\right) \\
+\mathbf{b}\left(\left(\boldsymbol{\sigma}_{\mathrm{S}}, \mathbf{u}_{\mathrm{D}}\right),(\boldsymbol{\psi}, \xi)\right)-\mathbf{c}((\boldsymbol{\varphi}, \lambda),(\boldsymbol{\psi}, \xi)),
\end{gathered}
$$


with

$$
\begin{gathered}
\mathbf{a}\left(\left(\boldsymbol{\sigma}_{\mathrm{S}}, \mathbf{u}_{\mathrm{D}}\right),\left(\boldsymbol{\tau}_{\mathrm{S}}, \mathbf{v}_{\mathrm{D}}\right)\right):=\nu^{-1}\left(\boldsymbol{\sigma}_{\mathrm{S}}^{d}, \boldsymbol{\tau}_{\mathrm{S}}^{d}\right)_{\mathrm{S}}+\left(\mathbf{K}^{-1} \mathbf{u}_{\mathrm{D}}, \mathbf{v}_{\mathrm{D}}\right)_{\mathrm{D}}, \\
{[\mathrm{A}+\mathrm{F}]} \\
\mathbf{b}\left(\left(\boldsymbol{\tau}_{\mathrm{S}}, \mathbf{v}_{\mathrm{D}}\right),(\boldsymbol{\psi}, \xi)\right):=\left\langle\boldsymbol{\tau}_{\mathrm{S}} \mathbf{n}, \boldsymbol{\psi}\right\rangle_{\Sigma}-\left\langle\mathbf{v}_{\mathrm{D}} \cdot \mathbf{n}, \xi\right\rangle_{\Sigma}, \\
{[\mathrm{C}+\mathrm{H}]} \\
\mathbf{c}((\boldsymbol{\varphi}, \lambda),(\boldsymbol{\psi}, \xi)):=\nu \kappa^{-1}\langle\boldsymbol{\varphi} \cdot \mathbf{t}, \boldsymbol{\psi} \cdot \mathbf{t}\rangle_{\Sigma}+\langle\boldsymbol{\varphi} \cdot \mathbf{n}, \xi\rangle_{\Sigma}-\langle\boldsymbol{\psi} \cdot \mathbf{n}, \lambda\rangle_{\Sigma} \\
{\left[\mathrm{D}+\mathrm{E}-\mathrm{E}^{\mathrm{t}}\right],}
\end{gathered}
$$

and

$$
\mathcal{B}(\underline{\boldsymbol{\tau}}, \underline{\mathbf{v}}):=\left(\operatorname{div} \boldsymbol{\tau}_{\mathrm{S}}, \mathbf{v}_{\mathrm{S}}\right)_{\mathrm{S}}-\left(\operatorname{div} \mathbf{v}_{\mathrm{D}}, q_{\mathrm{D}}\right)_{\mathrm{D}}+\eta\langle\boldsymbol{\psi} \cdot \mathbf{n}, 1\rangle_{\sigma} \quad[\mathrm{B}+\mathrm{G}+\mathrm{J}] .
$$

It is quite evident from (2.29) that $\mathcal{A}$ has a mixed structure with penalization term given by $\mathbf{- c}$, which confirms the doubly-mixed character of (2.27). Note also that $\mathbf{c}$ is nonsymmetric and positive semidefinite (this fact will be emphasized and utilized in Section (3). In addition, we remark again that the diagonal character of the bilinear forms $\mathbf{a}, \mathbf{b}$, and $\mathcal{B}$ will yield simpler and more straightforward proofs of the corresponding inf-sup conditions.

\section{Analysis of the Continuous problem}

The approach that we will follow for the analysis of the continuous problem (2.27) is the one of Fredholm theorems and Babuška-Brezzi theory for mixed problems.

3.1. Preliminaries. We group here some merely technical results and further notations that will serve for the forthcoming analysis. For elementary results on Hilbert space theory, we refer to [17] for example. The first of them is an abstract result on Hilbert spaces that can be read as follows: a symmetric positive definite bilinear form in a Hilbert space that can be made elliptic by the addition of a compact bilinear form, is necessarily elliptic.

Lemma 3.1. Let $X$ be a Hilbert space, and let $a: X \times X \rightarrow \mathbb{R}$ and $k: X \times X \rightarrow \mathbb{R}$ be bounded bilinear forms. Assume that $a$ is symmetric and positive definite, $k$ is compact, and there exists $\alpha>0$ such that

$$
a(x, x)+k(x, x) \geq \alpha\|x\|^{2} \quad \forall x \in X .
$$

Then there exists $\beta>0$ such that

$$
a(x, x) \geq \beta\|x\|^{2} \quad \forall x \in X .
$$

Proof. Let $A: X \rightarrow X^{\prime}$ and $K: X \rightarrow X^{\prime}$ be the linear and bounded operators induced by $a$ and $k$, respectively, that is, $A(x)=a(x, \cdot)$ and $K(x)=k(x, \cdot)$ for each $x \in X$. The hypotheses on $a$ and $k$ imply that $A$ is selfadjoint and injective, $K$ is compact, and $A+K$ is invertible, whence $A$ is Fredholm of index zero. It follows that $A$ is an invertible selfadjoint positive definite operator, and hence, by elementary spectral properties of bounded selfadjoint operators, $A$ is necessarily elliptic.

Lemma 3.2. There exists $c>0$ such that

$$
\left\|\mathbf{v}_{\mathrm{D}}\right\|_{0, \Omega_{\mathrm{D}}} \geq c\left\|\mathbf{v}_{\mathrm{D}}\right\|_{\operatorname{div}, \Omega_{\mathrm{D}}} \quad \forall \mathbf{v}_{\mathrm{D}} \in \mathbf{H}\left(\operatorname{div} ; \Omega_{\mathrm{D}}\right) \quad \text { such that } \operatorname{div} \mathbf{v}_{\mathrm{D}} \in \mathbb{P}_{0}\left(\Omega_{\mathrm{D}}\right) .
$$


Proof. Let $\mathbf{v}_{\mathrm{D}} \in \mathbf{H}\left(\operatorname{div} ; \Omega_{\mathrm{D}}\right)$ such that $\operatorname{div} \mathbf{v}_{\mathrm{D}} \in \mathbb{P}_{0}\left(\Omega_{\mathrm{D}}\right)$. It is simple to see that

$$
\left\|\mathbf{v}_{\mathrm{D}}\right\|_{\text {div }, \Omega_{\mathrm{D}}}^{2}=\left\|\mathbf{v}_{\mathrm{D}}\right\|_{0, \Omega_{\mathrm{D}}}^{2}+k\left(\mathbf{v}_{\mathrm{D}}, \mathbf{v}_{\mathrm{D}}\right)
$$

where $k: \mathbf{H}\left(\operatorname{div} ; \Omega_{\mathrm{D}}\right) \times \mathbf{H}\left(\operatorname{div} ; \Omega_{\mathrm{D}}\right) \rightarrow \mathbb{R}$ is the bounded bilinear form defined by

$$
k\left(\mathbf{w}_{\mathrm{D}}, \mathbf{v}_{\mathrm{D}}\right):=\frac{1}{\left|\Omega_{\mathrm{D}}\right|}\left\{\int_{\Omega_{\mathrm{D}}} \operatorname{div} \mathbf{w}_{\mathrm{D}}\right\}\left\{\int_{\Omega_{\mathrm{D}}} \operatorname{div} \mathbf{v}_{\mathrm{D}}\right\} \quad \forall \mathbf{w}_{\mathrm{D}}, \mathbf{v}_{\mathrm{D}} \in \mathbf{H}\left(\operatorname{div} ; \Omega_{\mathrm{D}}\right) .
$$

Since $k$ is clearly compact, a direct application of Lemma 3.1 ends the proof.

Lemma 3.3. There exists $c_{1}>0$ such that

$$
\left\|\boldsymbol{\tau}_{\mathrm{S}}^{d}\right\|_{0, \Omega_{\mathrm{S}}}^{2}+\left\|\operatorname{div} \boldsymbol{\tau}_{\mathrm{S}}\right\|_{0, \Omega_{\mathrm{S}}}^{2} \geq c_{1}\left\|\boldsymbol{\tau}_{\mathrm{S}}\right\|_{0, \Omega_{\mathrm{S}}}^{2} \quad \forall \boldsymbol{\tau}_{\mathrm{S}} \in \mathbb{H}_{0}\left(\operatorname{div} ; \Omega_{\mathrm{S}}\right)
$$

Proof. See [3, Lemma 3.1] or [8, Proposition 3.1, Chapter IV].

Lemma 3.4. Let $\left(X,\langle\cdot, \cdot\rangle_{X}\right)$ and $\left.Y,\langle\cdot, \cdot\rangle_{Y}\right)$ be Hilbert spaces and let $A: X \rightarrow X$, $B: X \rightarrow Y$, and $C: Y \rightarrow Y$ be bounded linear operators. Assume that $A$ is elliptic, $B$ is surjective, and $C$ is positive semidefinite, that is, respectively,

i) there exists $\alpha>0$ such that $\langle A(x), x\rangle_{X} \geq \alpha\|x\|_{X}^{2} \quad \forall x \in X$,

ii) there exists $\beta>0$ such that $\left\|B^{*}(y)\right\|_{X} \geq \beta\|y\|_{Y} \quad \forall y \in Y$,

iii) $\langle C(y), y\rangle_{Y} \geq 0 \quad \forall y \in Y$.

Then the matrix operator $T:=\left[\begin{array}{cc}A & B^{*} \\ B & -C\end{array}\right]: X \times Y \rightarrow X \times Y$ is bijective.

Proof. It suffices to observe that, being $A$ invertible thanks to i), $T$ is bijective if and only if $S:=B A^{-1} B^{*}+C: Y \rightarrow Y$ is bijective, which follows from the fact that $S$ becomes elliptic. We omit further details and refer to [19, Lemma 2.1] for a nonlinear version of this result.

We end this section with some notation concerning our product spaces. In fact, we now let

$$
\mathbb{X}:=\mathbb{H}\left(\operatorname{div} ; \Omega_{\mathrm{S}}\right) \times \mathbf{H}\left(\operatorname{div} ; \Omega_{\mathrm{D}}\right) \times \mathbf{H}^{1 / 2}(\Sigma) \times H^{1 / 2}(\Sigma),
$$

recall that $\mathbb{M}:=\mathbf{L}^{2}\left(\Omega_{\mathrm{S}}\right) \times L^{2}\left(\Omega_{\mathrm{D}}\right) \times \mathbb{R}($ cf. (2.26) $)$, and define

$\|\underline{\boldsymbol{\tau}}\|_{\mathbb{X}}:=\left\|\boldsymbol{\tau}_{\mathrm{S}}\right\|_{\operatorname{div}, \Omega_{\mathrm{S}}}+\left\|\mathbf{v}_{\mathrm{D}}\right\|_{\operatorname{div}, \Omega_{\mathrm{D}}}+\|\boldsymbol{\psi}\|_{1 / 2, \Sigma}+\|\xi\|_{1 / 2, \Sigma} \quad \forall \underline{\boldsymbol{\tau}}:=\left(\boldsymbol{\tau}_{\mathrm{S}}, \mathbf{v}_{\mathrm{D}}, \boldsymbol{\psi}, \xi\right) \in \mathbb{X}$

and

$$
\|\underline{\mathbf{v}}\|_{\mathbb{M}}:=\left\|\mathbf{v}_{\mathrm{S}}\right\|_{0, \Omega_{\mathrm{S}}}+\left\|q_{\mathrm{D}}\right\|_{0, \Omega_{\mathrm{D}}}+|\eta| \quad \forall \underline{\mathbf{v}}:=\left(\mathbf{v}_{\mathrm{S}}, q_{\mathrm{D}}, \eta\right) \in \mathbb{M} .
$$

Note that $\|\cdot\|_{\mathbb{X}}$ and $\|\cdot\|_{\mathbb{M}}$ are equivalent to the product norms that make $\mathbb{X}$ and $\mathbb{M}$ (and hence $\mathbb{X}_{0}$ and $\mathbb{M}_{0}$ ) Hilbert spaces. We will use them for all forthcoming estimates.

3.2. The main results. We begin by showing that (2.27) has a one-dimensional kernel. More precisely, we have the following result.

Lemma 3.5. Let $(\underline{\boldsymbol{\sigma}}, \underline{\mathbf{u}}):=\left(\left(\boldsymbol{\sigma}_{\mathrm{S}}, \mathbf{u}_{\mathrm{D}}, \boldsymbol{\varphi}, \lambda\right),\left(\mathbf{u}_{\mathrm{S}}, p_{\mathrm{D}}, \mu\right)\right) \in \mathbb{X}_{0} \times \mathbb{M}$ be a solution of (2.27) with homogeneous right-hand side. Then there exists $c \in \mathbb{R}$ such that

$$
\underline{\boldsymbol{\sigma}}=(\mathbf{0}, \mathbf{0}, \mathbf{0}, c) \text {. and } \underline{\mathbf{u}}=(\mathbf{0}, c,-c) .
$$


Proof. Testing the equations (2.27) with the vectors $\underline{\boldsymbol{\tau}}=\left(\boldsymbol{\sigma}_{\mathrm{S}}, \mathbf{u}_{\mathrm{D}},-\boldsymbol{\varphi},-\lambda\right)$ and $\underline{\mathbf{v}}=\left(-\mathbf{u}_{\mathrm{S}},-p_{\mathrm{D}}, \mu\right)$, and then adding them, we find that

$$
0=\nu^{-1}\left(\boldsymbol{\sigma}_{\mathrm{S}}^{d}, \boldsymbol{\sigma}_{\mathrm{S}}^{d}\right)_{\mathrm{S}}+\left(\mathrm{K}^{-1} \mathbf{u}_{\mathrm{D}}, \mathbf{u}_{\mathrm{D}}\right)_{\mathrm{D}}+\nu \kappa^{-1}\langle\boldsymbol{\varphi} \cdot \mathbf{t}, \boldsymbol{\varphi} \cdot \mathbf{t}\rangle_{\Sigma} .
$$

Note that this is equivalent to changing the sign of either the second and third rows in ((1)) or all the rows but the first two in ((2)) or all the rows but the first two and the last one in ((3)) (see Table 1), and then adding all of them. It is clear from the above equation that

$$
\boldsymbol{\sigma}_{\mathrm{S}}^{d}=\mathbf{0} \quad \text { in } \Omega_{\mathrm{S}}, \quad \mathbf{u}_{\mathrm{D}}=\mathbf{0} \quad \text { in } \Omega_{\mathrm{D}}, \quad \text { and } \boldsymbol{\varphi} \cdot \mathbf{t}=0 \text { on } \Sigma .
$$

Using Theorem 2.1 it follows that $\nabla \mathbf{u}_{\mathrm{S}}=\nu^{-1} \boldsymbol{\sigma}_{\mathrm{S}}^{d}=\mathbf{0}\left(\mathrm{cf}\right.$. (2.5)) and $-\mathbf{u}_{\mathrm{S}} \cdot \mathbf{t}=0$, which implies that $\mathbf{u}_{\mathrm{S}}=\mathbf{0}$ in $\Omega_{\mathrm{S}}$. Hence, again by Theorem 2.1 we have that $\boldsymbol{\varphi}=\mathbf{0}$ and $\operatorname{div} \boldsymbol{\sigma}_{\mathrm{S}}=\mathbf{0}$, which, together with the fact that $\boldsymbol{\sigma}_{\mathrm{S}} \in \mathbb{H}_{0}\left(\operatorname{div} ; \Omega_{\mathrm{S}}\right)$ and $\boldsymbol{\sigma}_{\mathrm{S}}^{d}=\mathbf{0}$, yields $\boldsymbol{\sigma}_{\mathrm{S}}=\mathbf{0}$ in $\Omega_{\mathrm{S}}$. Next, since $\nabla p_{\mathrm{D}}=\mathrm{K}^{-1} \mathbf{u}_{\mathrm{D}}=\mathbf{0}$, we deduce the existence of $c \in \mathbb{R}$ such that $p_{\mathrm{D}}=c$ in $\Omega_{\mathrm{D}}$, whence $\lambda=c$ on $\Sigma$. According to the above, the equation (2.22) reduces now to $\mu \mathbf{n}+c \mathbf{n}=\mathbf{0}$ on $\Sigma$, which gives $\mu=-c$.

Our next goal is to demonstrate that a simple restriction on the pressure in the Darcy domain solves the indetermination generated by the nonnull kernel of (2.27). To this end, we now let

$$
\mathbb{M}_{0}:=\mathbf{L}^{2}\left(\Omega_{\mathrm{S}}\right) \times L_{0}^{2}\left(\Omega_{\mathrm{D}}\right) \times \mathbb{R}
$$

where

$$
L_{0}^{2}\left(\Omega_{\mathrm{D}}\right):=\left\{q \in L^{2}\left(\Omega_{\mathrm{D}}\right): \quad \int_{\Omega_{\mathrm{D}}} q=0\right\},
$$

and consider the reduced problem: Find $(\underline{\boldsymbol{\sigma}}, \underline{\mathbf{u}}) \in \mathbb{X}_{0} \times \mathbb{M}_{0}$ such that

$$
\begin{array}{rlrl}
\mathcal{A}(\underline{\boldsymbol{\sigma}}, \underline{\boldsymbol{\tau}})+\mathcal{B}(\underline{\boldsymbol{\tau}}, \underline{\mathbf{u}}) & =\mathcal{F}(\underline{\boldsymbol{\tau}}) & \forall \underline{\boldsymbol{\tau}}:=\left(\boldsymbol{\tau}_{\mathrm{S}}, \mathbf{v}_{\mathrm{D}}, \boldsymbol{\psi}, \xi\right) \in \mathbb{X}_{0}, \\
\mathcal{B}(\underline{\boldsymbol{\sigma}}, \underline{\mathbf{v}}) & =\mathcal{G}(\underline{\mathbf{v}}) \quad \forall \underline{\mathbf{v}}:=\left(\mathbf{v}_{\mathrm{S}}, q_{\mathrm{D}}, \eta\right) \in \mathbb{M}_{0} .
\end{array}
$$

Throughout the rest of the section we follow the analysis suggested by the BabuškaBrezzi theory to conclude finally that $(3.2)$ is well posed. This requires the inf-sup condition for $\mathcal{B}$ and the invertibility of the operator induced by $\mathcal{A}$ in the kernel of $\mathcal{B}$. We begin with the first.

Lemma 3.6. There exists $\beta>0$ such that

$$
\sup _{\underline{\boldsymbol{\tau}} \in \mathbb{X}_{0} \backslash \mathbf{0}} \frac{\mathcal{B}(\underline{\boldsymbol{\tau}}, \underline{\mathbf{v}})}{\|\underline{\boldsymbol{\tau}}\|_{\mathbb{X}}} \geq \beta\|\underline{\mathbf{v}}\|_{\mathbb{M}} \quad \forall \underline{\mathbf{v}} \in \mathbb{M}_{0} .
$$

Proof. We first observe that the diagonal character of $\mathcal{B}$ (cf. (2.30) ) guarantees that (3.3) is equivalent to the following three independent inf-sup conditions:

$$
\begin{aligned}
& \sup _{\boldsymbol{\tau}_{\mathrm{S}} \in \mathbb{H}_{0}\left(\mathbf{d i v} ; \Omega_{\mathrm{S}}\right) \backslash \mathbf{0}} \frac{\left(\operatorname{div} \boldsymbol{\tau}_{\mathrm{S}}, \mathbf{v}_{\mathrm{S}}\right)_{\mathrm{S}}}{\left\|\boldsymbol{\tau}_{\mathrm{S}}\right\|_{\operatorname{div}, \Omega_{\mathrm{S}}}} \geq \beta_{\mathrm{S}}\left\|\mathbf{v}_{\mathrm{S}}\right\|_{0, \Omega_{\mathrm{S}}} \quad \forall \mathbf{v}_{\mathrm{S}} \in \mathbf{L}^{2}\left(\Omega_{\mathrm{S}}\right), \\
& \sup _{\mathbf{v}_{\mathrm{D}} \in \mathbf{H}\left(\operatorname{div} ; \Omega_{\mathrm{D}}\right) \backslash \mathbf{0}} \frac{\left(\operatorname{div} \mathbf{v}_{\mathrm{D}}, q_{\mathrm{D}}\right)_{\mathrm{D}}}{\left\|\mathbf{v}_{\mathrm{D}}\right\|_{\operatorname{div}, \Omega_{\mathrm{D}}}} \geq \beta_{\mathrm{D}}\left\|q_{\mathrm{D}}\right\|_{0, \Omega_{\mathrm{D}}} \quad \forall q_{\mathrm{D}} \in L_{0}^{2}\left(\Omega_{\mathrm{D}}\right), \\
& \sup _{\boldsymbol{\psi} \in \mathbf{H}^{1 / 2}(\Sigma) \backslash \mathbf{0}} \frac{\eta\langle\boldsymbol{\psi} \cdot \mathbf{n}, 1\rangle_{\Sigma}}{\|\boldsymbol{\psi}\|_{1 / 2, \Sigma}} \geq \beta_{\Sigma}|\eta| \quad \forall \eta \in \mathbb{R},
\end{aligned}
$$


with $\beta_{\mathrm{S}}, \beta_{\mathrm{D}}, \beta_{\Sigma}>0$. For instance, the above statement follows from a direct application of the characterization result for the inf-sup condition on product spaces provided in [22, Theorem 5].

Now, given $\mathbf{v}_{\mathrm{S}} \in \mathbf{L}^{2}\left(\Omega_{\mathrm{S}}\right)$ we define $\boldsymbol{\tau}$ as the $\mathbb{H}_{0}\left(\mathbf{d i v} ; \Omega_{\mathrm{S}}\right)$-component of $\nabla \mathbf{z} \in$ $\mathbb{H}\left(\operatorname{div} ; \Omega_{\mathrm{S}}\right)$, where $\mathbf{z} \in \mathbf{H}^{1}\left(\Omega_{\mathrm{S}}\right)$ is the unique solution of the boundary value problem:

$$
\Delta \mathbf{z}=\mathbf{v}_{\mathrm{S}} \quad \text { in } \quad \Omega_{\mathrm{S}}, \quad \mathbf{z}=\mathbf{0} \quad \text { on } \partial \Omega_{\mathrm{S}} .
$$

This proves the surjectivity of the operator $\operatorname{div}: \mathbb{H}_{0}\left(\mathbf{d i v} ; \Omega_{\mathrm{S}}\right) \rightarrow \mathbf{L}^{2}\left(\Omega_{\mathrm{S}}\right)$, which is (3.4). Similarly, it is easy to see that $\operatorname{div}: \mathbf{H}\left(\operatorname{div} ; \Omega_{\mathrm{D}}\right) \rightarrow L^{2}\left(\Omega_{\mathrm{D}}\right)$ is also surjective, which yields (3.5).

On the other hand, the inf-sup condition (3.6) is equivalent to the surjectivity of the operator $\boldsymbol{\psi} \rightarrow\langle\boldsymbol{\psi} \cdot \mathbf{n}, 1\rangle_{\Sigma}$ from $\mathbf{H}^{1 / 2}(\Sigma)$ to $\mathbb{R}$, which in turn is equivalent to showing the existence of $\boldsymbol{\psi}_{0} \in \mathbf{H}^{1 / 2}(\Sigma)$ such that $\left\langle\boldsymbol{\psi}_{0} \cdot \mathbf{n}, 1\right\rangle_{\Sigma} \neq 0$. In fact, we pick one corner point of $\Sigma$ and define a function $v$ that is continuous, linear on each side of $\Sigma$, equal to one in the chosen vertex, and zero on all other ones. If $\mathbf{n}_{1}$ and $\mathbf{n}_{2}$ are the normal vectors on the two sides of $\Sigma$ that meet at the corner point, then $\boldsymbol{\psi}_{0}:=v\left(\mathbf{n}_{1}+\mathbf{n}_{2}\right)$ satisfies the required property.

We now let $\mathbb{V}$ be the kernel of $\mathcal{B}$, that is,

$$
\mathbb{V}:=\left\{\underline{\boldsymbol{\tau}} \in \mathbb{X}_{0}: \quad \mathcal{B}(\underline{\boldsymbol{\tau}}, \underline{\mathbf{v}})=0 \quad \forall \underline{\mathbf{v}} \in \mathbb{M}_{0}\right\}
$$

It is easy to see from the definition of $\mathcal{B}($ cf. (2.30) $)$ that $\mathbb{V}=\mathbb{V}_{1} \times \mathbb{V}_{2}$, where

$$
\mathbb{V}_{1}=\tilde{\mathbb{H}}_{0}\left(\operatorname{div} ; \Omega_{\mathrm{S}}\right) \times \tilde{\mathbf{H}}\left(\operatorname{div} ; \Omega_{\mathrm{D}}\right) \text { and } \mathbb{V}_{2}=\tilde{\mathbf{H}}^{1 / 2}(\Sigma) \times H^{1 / 2}(\Sigma)
$$

with

$$
\begin{gathered}
\tilde{\mathbb{H}}_{0}\left(\operatorname{div} ; \Omega_{\mathrm{S}}\right):=\left\{\boldsymbol{\tau}_{\mathrm{S}} \in \mathbb{H}_{0}\left(\operatorname{div} ; \Omega_{\mathrm{S}}\right): \quad \operatorname{div} \boldsymbol{\tau}_{\mathrm{S}}=\mathbf{0}\right\} \\
\tilde{\mathbf{H}}\left(\operatorname{div} ; \Omega_{\mathrm{D}}\right):=\left\{\mathbf{v}_{\mathrm{D}} \in \mathbf{H}\left(\operatorname{div} ; \Omega_{\mathrm{D}}\right): \quad \operatorname{div} \mathbf{v}_{\mathrm{D}} \in \mathbb{P}_{0}\left(\Omega_{\mathrm{D}}\right)\right\}
\end{gathered}
$$

and

$$
\tilde{\mathbf{H}}^{1 / 2}(\Sigma):=\left\{\boldsymbol{\psi} \in \mathbf{H}^{1 / 2}(\Sigma):\langle\boldsymbol{\psi} \cdot \mathbf{n}, 1\rangle_{\Sigma}=0\right\}
$$

Then, in what follows we apply Lemma 3.4 to prove that the operator induced by $\mathcal{A}$ (cf. (2.29) $)$ is invertible in $\mathbb{V}$. This means showing that $\mathbf{a}$ is elliptic on $\mathbb{V}_{1}, \mathbf{b}$ satisfies the inf-sup condition on $\mathbb{V}_{1} \times \mathbb{V}_{2}$, and $\mathbf{c}$ is positive semidefinite on $\mathbb{V}_{2}$.

As remarked in Section 2, the condition on $\mathbf{c}$ is pretty straightforward since

$$
\mathbf{c}((\boldsymbol{\varphi}, \lambda),(\boldsymbol{\varphi}, \lambda))=\nu \kappa^{-1}\|\boldsymbol{\varphi} \cdot \mathbf{t}\|_{0, \Sigma}^{2} \geq 0 \quad \forall(\boldsymbol{\varphi}, \lambda) \in \mathbf{H}^{1 / 2}(\Sigma) \times H^{1 / 2}(\Sigma) .
$$

The remaining conditions for $\mathbf{a}$ and $\mathbf{b}$ are established in the following lemmas.

Lemma 3.7. There exists $\alpha_{1}>0$ such that for each $\left(\boldsymbol{\tau}_{\mathrm{S}}, \mathbf{v}_{\mathrm{D}}\right) \in \mathbb{V}_{1}$ there holds

$$
\mathbf{a}\left(\left(\boldsymbol{\tau}_{\mathrm{S}}, \mathbf{v}_{\mathrm{D}}\right),\left(\boldsymbol{\tau}_{\mathrm{S}}, \mathbf{v}_{\mathrm{D}}\right)\right) \geq \alpha_{1}\left\{\left\|\boldsymbol{\tau}_{\mathrm{S}}\right\|_{\operatorname{div}, \Omega_{\mathrm{S}}}^{2}+\left\|\mathbf{v}_{\mathrm{D}}\right\|_{\operatorname{div}, \Omega_{\mathrm{D}}}^{2}\right\} .
$$

Proof. It suffices to observe that

$$
\begin{aligned}
\mathbf{a}\left(\left(\boldsymbol{\tau}_{\mathrm{S}}, \mathbf{v}_{\mathrm{D}}\right),\left(\boldsymbol{\tau}_{\mathrm{S}}, \mathbf{v}_{\mathrm{D}}\right)\right) & =\nu^{-1}\left\|\boldsymbol{\tau}_{\mathrm{S}}^{d}\right\|_{0, \Omega_{\mathrm{S}}}^{2}+\left(\mathbf{K}^{-1} \mathbf{v}_{\mathrm{D}}, \mathbf{v}_{\mathrm{D}}\right)_{\mathrm{D}} \\
& \geq c\left\{\left\|\boldsymbol{\tau}_{\mathrm{S}}^{d}\right\|_{0, \Omega_{\mathrm{S}}}^{2}+\left\|\mathbf{v}_{\mathrm{D}}\right\|_{0, \Omega_{\mathrm{D}}}^{2}\right\},
\end{aligned}
$$

and then apply Lemmas 3.3 and 3.2 
Lemma 3.8. There exists $\tilde{\beta}>0$ such that

$$
\sup _{\left(\boldsymbol{\tau}_{\mathrm{S}}, \mathbf{v}_{\mathrm{D}}\right) \in \mathbb{V}_{1} \backslash \mathbf{0}} \frac{\mathbf{b}\left(\left(\boldsymbol{\tau}_{\mathrm{S}}, \mathbf{v}_{\mathrm{D}}\right),(\boldsymbol{\psi}, \xi)\right)}{\left\|\left(\boldsymbol{\tau}_{\mathrm{S}}, \mathbf{v}_{\mathrm{D}}\right)\right\|} \geq \tilde{\beta}\|(\boldsymbol{\psi}, \xi)\| \quad \forall(\boldsymbol{\psi}, \xi) \in \mathbb{V}_{2} .
$$

Proof. Analogously to the proof of Lemma 3.6, and thanks to the diagonal character of $\mathbf{b}$, we find that (3.8) is equivalent to the following two independent inequalities:

$$
\begin{aligned}
\sup _{\boldsymbol{\tau}_{\mathrm{S}} \in \tilde{\mathbb{H}}_{0}\left(\operatorname{div} ; \Omega_{\mathrm{S}}\right) \backslash \mathbf{0}} \frac{\left\langle\boldsymbol{\tau}_{\mathrm{S}} \mathbf{n}, \boldsymbol{\psi}\right\rangle_{\Sigma}}{\left\|\boldsymbol{\tau}_{\mathrm{S}}\right\|_{\operatorname{div}, \Omega_{\mathrm{S}}}} \geq \tilde{\beta}_{\mathrm{S}}\|\boldsymbol{\psi}\|_{1 / 2, \Sigma} \quad \forall \boldsymbol{\psi} \in \tilde{\mathbf{H}}^{1 / 2}(\Sigma), \\
\sup _{\mathbf{v}_{\mathrm{D}} \in \tilde{\mathbf{H}}\left(\operatorname{div} ; \Omega_{\mathrm{D}}\right) \backslash \mathbf{0}} \frac{\left\langle\mathbf{v}_{\mathrm{D}} \cdot \mathbf{n}, \xi\right\rangle_{\Sigma}}{\left\|\mathbf{v}_{\mathrm{D}}\right\|_{\text {div }, \Omega_{\mathrm{D}}}} \geq \tilde{\beta}_{\mathrm{D}}\|\xi\|_{1 / 2, \Sigma} \quad \forall \xi \in H^{1 / 2}(\Sigma),
\end{aligned}
$$

with $\tilde{\beta}_{\mathrm{S}}, \tilde{\beta}_{\mathrm{D}}>0$.

Now, given $\chi \in \mathbf{H}^{-1 / 2}(\Sigma)$ we let $\boldsymbol{\tau}$ be the $\mathbb{H}_{0}\left(\operatorname{div} ; \Omega_{\mathrm{S}}\right)$-component of $\nabla \mathbf{z} \in$ $\mathbb{H}\left(\operatorname{div} ; \Omega_{\mathrm{S}}\right)$, where $\mathbf{z} \in \mathbf{H}^{1}\left(\Omega_{\mathrm{S}}\right)$ is the unique solution of the boundary value problem:

$$
\Delta \mathbf{z}=\mathbf{0} \quad \text { in } \quad \Omega_{\mathrm{S}}, \quad \mathbf{z}=\mathbf{0} \quad \text { on } \Gamma_{\mathrm{S}}, \quad \nabla \mathbf{z} \mathbf{n}=\chi \quad \text { on } \Sigma .
$$

In other words, $\boldsymbol{\tau}:=\nabla \mathbf{z}-c \mathrm{I}$, where $c:=\frac{1}{2\left|\Omega_{\mathrm{S}}\right|} \int_{\Omega_{\mathrm{S}}} \operatorname{tr} \nabla \mathbf{z}$ (cf. (2.10) $)$, which implies that $\boldsymbol{\tau} \in \tilde{\mathbb{H}}_{0}\left(\operatorname{div} ; \Omega_{\mathrm{S}}\right)$ and $\boldsymbol{\tau} \mathbf{n}=\boldsymbol{\chi}-c \mathbf{n}$ on $\Sigma$. It follows that $\langle\boldsymbol{\tau} \mathbf{n}, \boldsymbol{\psi}\rangle_{\Sigma}=\langle\boldsymbol{\chi}, \boldsymbol{\psi}\rangle_{\Sigma}$ for each $\boldsymbol{\psi} \in \tilde{\mathbf{H}}^{1 / 2}(\Sigma)$, which proves the surjectivity of the operator $\boldsymbol{\tau} \rightarrow \boldsymbol{\tau} \mathbf{n}$ from $\tilde{\mathbb{H}}_{0}\left(\operatorname{div} ; \Omega_{\mathrm{S}}\right)$ to $\left(\tilde{\mathbf{H}}^{1 / 2}(\Sigma)\right)^{\prime}$, that is, (3.9).

Similarly, given $\chi \in \mathrm{H}^{-1 / 2}(\Sigma)$ we define $\mathbf{v}:=\nabla z \in \mathbf{H}\left(\operatorname{div} ; \Omega_{\mathrm{D}}\right)$, where $z \in$ $H^{1}\left(\Omega_{\mathrm{D}}\right)$ is the unique solution of the boundary value problem:

$$
\Delta z=\frac{1}{\left|\Omega_{\mathrm{D}}\right|}\langle\chi, 1\rangle_{\Sigma} \quad \text { in } \quad \Omega_{\mathrm{D}}, \quad \nabla z \cdot \mathbf{n}=\chi \quad \text { on } \quad \Sigma, \quad \int_{\Omega_{\mathrm{D}}} z=0 .
$$

It follows that $\mathbf{v} \in \tilde{\mathbf{H}}\left(\operatorname{div} ; \Omega_{\mathrm{D}}\right)$ and $\mathbf{v} \cdot \mathbf{n}=\chi \quad$ on $\quad \Sigma$, which proves the surjectivity of the operator $\mathbf{v} \rightarrow \mathbf{v} \cdot \mathbf{n}$ from $\tilde{\mathbf{H}}\left(\operatorname{div} ; \Omega_{\mathrm{D}}\right)$ to $\mathrm{H}^{-1 / 2}(\Sigma)$, that is, (3.10).

As a consequence of the previous analysis we conclude that $\mathcal{A}$ is invertible in the kernel of $\mathcal{B}$. This result and the inf-sup condition for $\mathcal{B}$ (cf. Lemma 3.6) allow us to establish the following theorem.

Theorem 3.9. For each pair $(\mathcal{F}, \mathcal{G}) \in \mathbb{X}_{0}^{\prime} \times \mathbb{M}_{0}^{\prime}$ there exists a unique $(\underline{\boldsymbol{\sigma}}, \underline{\mathbf{u}}) \in$ $\mathbb{X}_{0} \times \mathbb{M}_{0}$ solution to (3.2), and there exists a constant $C>0$, independent of the solution, such that

$$
\|(\underline{\boldsymbol{\sigma}}, \underline{\mathbf{u}})\|_{\mathbb{X} \times \mathbb{M}} \leq C\left\{\|\mathcal{F}\|_{\mathbb{X}_{0}^{\prime}}+\|\mathcal{G}\|_{\mathbb{M}_{0}^{\prime}}\right\} .
$$

In particular, if $(\mathcal{F}, \mathcal{G})$ is given by (2.28) and there holds $\int_{\Omega_{\mathrm{D}}} f_{\mathrm{D}}=0($ cf. (2.3) $)$, then the solution of (3.2) is also a solution of the original variational formulation (2.27).

Proof. The well posedness of (3.2) follows from a straightforward application of the classical Babuška-Brezzi theory for mixed problems (see, e.g. 23, Theorem I.4.1] or [8, Chapter II]). Now, let $(\underline{\boldsymbol{\sigma}}, \underline{\mathbf{u}}) \in \mathbb{X}_{0} \times \mathbb{M}_{0}$ be the solution of (3.2) with $(\mathcal{F}, \mathcal{G})$ given by (2.28). Since the first equations of (2.27) and (3.2) coincide, it only remains to show that $\boldsymbol{\sigma}$ verifies the second equation of (2.27) to conclude that 
$(\underline{\boldsymbol{\sigma}}, \underline{\mathbf{u}})$ also solves that problem. In fact, taking $\underline{\boldsymbol{\tau}}=(\mathbf{0}, \mathbf{0}, \mathbf{0}, \xi)$ in the first equation of (3.2) we deduce that $\mathbf{u}_{\mathrm{D}} \cdot \mathbf{n}+\boldsymbol{\varphi} \cdot \mathbf{n}=0$ on $\Sigma$, and hence, according to the definition of $\mathcal{B}$ (cf. (2.30) ) and the second equation of (3.2), we obtain that

$$
\begin{gathered}
\mathcal{B}(\underline{\boldsymbol{\sigma}},(\mathbf{0}, 1,0))=-\left(\operatorname{div} \mathbf{u}_{\mathrm{D}}, 1\right)_{\mathrm{D}}=\left\langle\mathbf{u}_{\mathrm{D}} \cdot \mathbf{n}, 1\right\rangle_{\Sigma}=-\langle\boldsymbol{\varphi} \cdot \mathbf{n}, 1\rangle_{\Sigma} \\
=\mathcal{B}(\underline{\boldsymbol{\sigma}},(\mathbf{0}, 0,-1))=\mathcal{G}((\mathbf{0}, 0,-1))=0 .
\end{gathered}
$$

Then, given $\underline{\mathbf{v}}=\left(\mathbf{v}_{\mathrm{S}}, q_{\mathrm{D}}, \eta\right) \in \mathbb{M}$, where $q_{\mathrm{D}}=q_{0}+c$, with $\left(q_{0}, c\right) \in L_{0}^{2}\left(\Omega_{\mathrm{D}}\right) \times \mathbb{R}$, we use the above identity and again the second equation of (3.2), to find that

$$
\begin{gathered}
\mathcal{B}(\underline{\boldsymbol{\sigma}}, \underline{\mathbf{v}})=\mathcal{B}\left(\underline{\boldsymbol{\sigma}},\left(\mathbf{v}_{\mathrm{S}}, q_{0}, \eta\right)\right)=\mathcal{G}\left(\left(\mathbf{v}_{\mathrm{S}}, q_{0}, \eta\right)\right)=-\left(\mathbf{f}_{\mathrm{S}}, \mathbf{v}_{\mathrm{S}}\right)_{\mathrm{S}}-\left(f_{\mathrm{D}}, q_{0}\right)_{\mathrm{D}} \\
=-\left(\mathbf{f}_{\mathrm{S}}, \mathbf{v}_{\mathrm{S}}\right)_{\mathrm{S}}-\left(f_{\mathrm{D}}-\frac{1}{\left|\Omega_{\mathrm{D}}\right|} \int_{\Omega_{\mathrm{D}}} f_{\mathrm{D}}, q_{\mathrm{D}}\right)_{\mathrm{D}},
\end{gathered}
$$

which, thanks to the assumption (2.3) , becomes $\mathcal{B}(\underline{\boldsymbol{\sigma}}, \underline{\mathbf{v}})=\mathcal{G}(\underline{\mathbf{v}}) \quad \forall \underline{\mathbf{v}} \in \mathbb{M}$.

Note from the last identity in the previous proof that if we solve (3.2) with $(\mathcal{F}, \mathcal{G})$ given by (2.28) but (2.3) is not satisfied, then we are finding a solution of (2.27) for a slightly modified right-hand side, with $\mathbf{f}_{\mathrm{S}}$ unchanged but with $f_{\mathrm{D}}-\frac{1}{\left|\Omega_{\mathrm{D}}\right|} \int_{\Omega_{\mathrm{D}}} f_{\mathrm{D}}$ instead of $f_{\mathrm{D}}$. Moreover, we can actually prove the following result characterizing the solvability of (2.27).

Theorem 3.10. Problem (2.27) with $(\mathcal{F}, \mathcal{G})$ given by (2.28) is solvable if and only if (2.3) holds. In that case, the solution is defined up to a multiple of the vector $((\mathbf{0}, \mathbf{0}, \mathbf{0}, 1),(\mathbf{0}, 1,-1))$.

Proof. It suffices to observe that the operator induced by the left-hand side of (2.27), say $\mathcal{L}$, is Fredholm of index zero. In fact, using that $L^{2}\left(\Omega_{\mathrm{D}}\right)=L_{0}^{2}\left(\Omega_{\mathrm{D}}\right) \oplus$ $\mathbb{P}_{0}\left(\Omega_{\mathrm{D}}\right)$, we decompose the pressure unknown $p_{\mathrm{D}}$ in (2.27) as $p_{\mathrm{D}}=p_{0}+c$ with $p_{0} \in L_{0}^{2}\left(\Omega_{\mathrm{D}}\right)$ and $c \in \mathbb{P}_{0}\left(\Omega_{\mathrm{D}}\right)$, and similarly for the corresponding test functions $q_{\mathrm{D}} \in L^{2}\left(\Omega_{\mathrm{D}}\right)$. In this way, it is easy to realize that (2.27) is equivalent to a compact perturbation of a problem equivalent to (3.2). Since the latter is well posed, this proves the announced property of $\mathcal{L}$. Now, the kernel of the adjoint operator $\mathcal{L}^{*}$ is the same as $\mathcal{L}$ because this operator is symmetric up to some sign changes of its rows (see Table 11). Therefore, by the Fredholm alternative, the system (2.27) is solvable if and only if the right-hand side vanishes when applied to an element of the kernel of the adjoint. With the right-hand side (2.28) and the kernel given in Lemma 3.5 this is just condition (2.3).

At this point we remark that the above analysis also applies when the fluid lies over the porous medium and the additional Neumann boundary condition (2.6) is incorporated into the model (as described at the end of Section 2.1). In particular, it is easy to see that (2.3) and its equivalence with the solvability of the original formulation (2.27) remain unchanged in this case. On the other hand, if we assume (2.7) instead of (2.6), the condition (2.3) does not hold any longer and the solvability analysis of (2.27) becomes simpler. Indeed, following the same arguments of the proof of Lemma 3.5. we find now, thanks to the fact that $\nabla p_{\mathrm{D}}=\mathbf{0}$ in $\Omega_{\mathrm{D}}$ and $p_{\mathrm{D}}=0$ on $\Gamma$, that $p_{\mathrm{D}}=0$ in $\Omega_{\mathrm{D}}$, which leads to a trivial kernel for (2.27). In other words, there is no need of incorporating any further restriction on the pressure $p_{\mathrm{D}}$ and the subsequent reduced problem (3.2) since the homogeneous Dirichlet boundary condition (2.7) already insures the uniqueness of solution. Consequently, 
up to minor modifications, the solvability analysis of (2.27) becomes very similar to the corresponding analysis of the present formulation (3.2).

\section{The Galerkin SCHEME}

In this section we introduce and analyze the Galerkin scheme of the reduced problem (3.2).

4.1. Preliminaries. Here we define the discrete system and establish suitable assumptions on the finite element subspaces ensuring later on that it becomes well posed. For this purpose, we first select two collections of discrete spaces:

$$
\begin{aligned}
\mathbf{H}_{h}\left(\Omega_{\mathrm{D}}\right) \subseteq \mathbf{H}\left(\operatorname{div} ; \Omega_{\mathrm{D}}\right), & L_{h}\left(\Omega_{\mathrm{D}}\right) \subseteq L^{2}\left(\Omega_{\mathrm{D}}\right), & & \Lambda_{h}^{\mathrm{D}}(\Sigma) \subseteq H^{1 / 2}(\Sigma), \\
\mathbf{H}_{h}\left(\Omega_{\mathrm{S}}\right) \subseteq \mathbf{H}\left(\operatorname{div} ; \Omega_{\mathrm{S}}\right), & L_{h}\left(\Omega_{\mathrm{S}}\right) \subseteq L^{2}\left(\Omega_{\mathrm{S}}\right), & & \Lambda_{h}^{\mathrm{S}}(\Sigma) \subseteq H^{1 / 2}(\Sigma) .
\end{aligned}
$$

However, the spaces for the Stokes domain will have to be doubled. In particular, in the case of the matrix-valued unknown $\boldsymbol{\sigma}_{\mathrm{S}}$ we will consider the space of matrixvalued functions whose rows belong to $\mathbf{H}_{h}\left(\Omega_{\mathrm{S}}\right)$. According to this we now define

$$
\begin{gathered}
\mathbf{L}_{h}\left(\Omega_{\mathrm{S}}\right):=L_{h}\left(\Omega_{\mathrm{S}}\right) \times L_{h}\left(\Omega_{\mathrm{S}}\right), \quad \boldsymbol{\Lambda}_{h}^{\mathrm{S}}(\Sigma):=\Lambda_{h}^{\mathrm{S}}(\Sigma) \times \Lambda_{h}^{\mathrm{S}}(\Sigma), \\
\mathbb{H}_{h}\left(\Omega_{\mathrm{S}}\right):=\left\{\boldsymbol{\tau}: \Omega_{\mathrm{S}} \rightarrow \mathbb{R}^{2 \times 2}: \mathbf{c}^{t} \boldsymbol{\tau} \in \mathbf{H}_{h}\left(\Omega_{\mathrm{S}}\right) \forall \mathbf{c} \in \mathbb{R}^{2}\right\} \subseteq \mathbb{H}\left(\operatorname{div} ; \Omega_{\mathrm{S}}\right),
\end{gathered}
$$

and

$$
\mathbb{H}_{h, 0}\left(\Omega_{\mathrm{S}}\right):=\mathbb{H}_{h}\left(\Omega_{\mathrm{S}}\right) \cap \mathbb{H}_{0}\left(\operatorname{div} ; \Omega_{\mathrm{S}}\right) .
$$

In addition, in order to deal with the mean value condition of the Darcy pressure we define

$$
L_{h, 0}\left(\Omega_{\mathrm{D}}\right):=L_{h}\left(\Omega_{\mathrm{D}}\right) \cap L_{0}^{2}\left(\Omega_{\mathrm{D}}\right) .
$$

In this way, we define the global finite element subspaces as:

$$
\begin{aligned}
\mathbb{X}_{h, 0} & :=\mathbb{H}_{h, 0}\left(\Omega_{\mathrm{S}}\right) \times \mathbf{H}_{h}\left(\Omega_{\mathrm{D}}\right) \times \boldsymbol{\Lambda}_{h}^{\mathrm{S}}(\Sigma) \times \Lambda_{h}^{\mathrm{D}}(\Sigma), \\
\mathbb{M}_{h, 0} & :=\mathbf{L}_{h}\left(\Omega_{\mathrm{S}}\right) \times L_{h, 0}\left(\Omega_{\mathrm{D}}\right) \times \mathbb{R},
\end{aligned}
$$

and consider the following Galerkin scheme for (3.2): Find $\left(\underline{\boldsymbol{\sigma}}_{h}, \underline{\mathbf{u}}_{h}\right) \in \mathbb{X}_{h, 0} \times \mathbb{M}_{h, 0}$ such that

$$
\begin{aligned}
\mathcal{A}\left(\underline{\boldsymbol{\sigma}}_{h}, \underline{\boldsymbol{\tau}}_{h}\right)+\mathcal{B}\left(\underline{\boldsymbol{\tau}}_{h}, \underline{\mathbf{u}}_{h}\right) & =\mathcal{F}\left(\underline{\boldsymbol{\tau}}_{h}\right) \quad \forall \underline{\boldsymbol{\tau}}_{h} \in \mathbb{X}_{h, 0}, \\
\mathcal{B}\left(\underline{\boldsymbol{\sigma}}_{h}, \underline{\mathbf{v}}_{h}\right) & =\mathcal{G}\left(\underline{\mathbf{v}}_{h}\right) \quad \forall \underline{\mathbf{v}}_{h} \in \mathbb{M}_{h, 0} .
\end{aligned}
$$

Note that the different structures shown in Table 1 are inherited by the linear system associated to (4.7) once we have chosen bases for all the discrete spaces.

In what follows we derive general hypotheses on the spaces (4.1) that will allow us to show in Section 4.2 below that (4.7) is well posed. Our approach consists of adapting to the present discrete case the arguments employed in the analysis of the continuous problem, mainly those from the proofs of Lemmas 3.6, 3.7, and 3.8. We begin by observing that in order to have meaningful spaces $\mathbb{H}_{h, 0}\left(\Omega_{\mathrm{S}}\right)$ and $L_{h, 0}\left(\Omega_{\mathrm{D}}\right)$ (cf. (4.4) and (4.5)), we need to be able to eliminate multiples of the identity matrix from $\mathbb{H}_{h}\left(\Omega_{\mathrm{S}}\right)$ and constants polynomials from $L_{h}\left(\Omega_{\mathrm{D}}\right)$. This request is certainly satisfied if we assume that:

(H.0) $\left[\mathbb{P}_{0}\left(\Omega_{\mathrm{S}}\right)\right]^{2} \subseteq \mathbf{H}_{h}\left(\Omega_{\mathrm{S}}\right)$ and $\mathbb{P}_{0}\left(\Omega_{\mathrm{D}}\right) \subseteq L_{h}\left(\Omega_{\mathrm{D}}\right)$. 
We remark that the above hypothesis is only related to the ability of the spaces to deal with problems inherent to the kernel of (2.27). In particular, it follows that $\mathrm{I} \in \mathbb{H}_{h}\left(\Omega_{\mathrm{S}}\right)$ for all $h$, and hence there holds the decomposition:

$$
\mathbb{H}_{h}\left(\Omega_{\mathrm{S}}\right)=\mathbb{H}_{h, 0}\left(\Omega_{\mathrm{S}}\right) \oplus \mathbb{P}_{0}\left(\Omega_{\mathrm{S}}\right) \mathrm{I} .
$$

Next, following the same diagonal argument utilized in the proof of Lemma 3.6 . we deduce that $\mathcal{B}$ satisfies the discrete inf-sup condition uniformly on $\mathbb{X}_{h, 0} \times \mathbb{M}_{h, 0}$ if and only if there exist $\beta_{\mathrm{S}}, \beta_{\mathrm{D}}, \beta_{\Sigma}>0$, independent of $h$, such that

$$
\begin{array}{ll}
\sup _{\boldsymbol{\tau}_{h} \in \mathbb{H}_{h, 0}\left(\Omega_{\mathrm{S}}\right) \backslash \mathbf{0}} \frac{\left(\operatorname{div} \boldsymbol{\tau}_{h}, \mathbf{v}_{h}\right)_{\mathrm{S}}}{\left\|\boldsymbol{\tau}_{h}\right\|_{\operatorname{div}, \Omega_{\mathrm{S}}}} \geq \beta_{\mathrm{S}}\left\|\mathbf{v}_{h}\right\|_{0, \Omega_{\mathrm{S}}} & \forall \mathbf{v}_{h} \in \mathbf{L}_{h}\left(\Omega_{\mathrm{S}}\right), \\
\sup _{\mathbf{v}_{h} \in \mathbf{H}_{h}\left(\Omega_{\mathrm{D}}\right) \backslash \mathbf{0}} \frac{\left(\operatorname{div} \mathbf{v}_{h}, q_{h}\right)_{\mathrm{D}}}{\left\|\mathbf{v}_{h}\right\|_{\operatorname{div}, \Omega_{\mathrm{D}}}} \geq \beta_{\mathrm{D}}\left\|q_{h}\right\|_{0, \Omega_{\mathrm{D}}} & \forall q_{h} \in L_{h, 0}\left(\Omega_{\mathrm{D}}\right), \\
\sup _{\boldsymbol{\psi}_{h} \in \boldsymbol{\Lambda}_{h}^{\mathrm{S}}(\Sigma) \backslash \mathbf{0}} \frac{\eta\left\langle\boldsymbol{\psi}_{h} \cdot \mathbf{n}, 1\right\rangle_{\Sigma}}{\left\|\boldsymbol{\psi}_{h}\right\|_{1 / 2, \Sigma}} \geq \beta_{\Sigma}|\eta| & \forall \eta \in \mathbb{R} .
\end{array}
$$

However, since $\operatorname{div} \mathbb{H}_{h}\left(\Omega_{\mathrm{S}}\right)=\operatorname{div} \mathbb{H}_{h, 0}\left(\Omega_{\mathrm{S}}\right)$ (cf. (4.8)), the supremum in (4.9) remains the same if taken on $\mathbb{H}_{h}\left(\Omega_{\mathrm{S}}\right)$ instead of $\mathbb{H}_{h, 0}\left(\Omega_{\mathrm{S}}\right)$, and hence this inequality turns out to be equivalent to the following inf-sup condition:

$$
\sup _{\tau_{h} \in \mathbf{H}_{h}\left(\Omega_{\mathrm{S}}\right) \backslash \mathbf{0}} \frac{\left(\operatorname{div} \tau_{h}, v_{h}\right)_{\mathrm{S}}}{\left\|\tau_{h}\right\|_{\text {div }, \Omega_{\mathrm{S}}}} \geq \beta_{\mathrm{S}}\left\|v_{h}\right\|_{0, \Omega_{\mathrm{S}}} \quad \forall v_{h} \in L_{h}\left(\Omega_{\mathrm{S}}\right) .
$$

Notice also that a sufficient condition for (4.11) is the existence of $\boldsymbol{\psi}_{0} \in \mathbf{H}^{1 / 2}(\Sigma)$ such that $\boldsymbol{\psi}_{0} \in \boldsymbol{\Lambda}_{h}^{\mathrm{S}}(\Sigma) \forall h$ and $\left\langle\boldsymbol{\psi}_{0} \cdot \mathbf{n}, 1\right\rangle_{\Sigma} \neq 0$. Consequently, we now introduce the following hypothesis summarizing the above analysis:

(H.1) There exist $\beta_{\mathrm{S}}, \beta_{\mathrm{D}}>0$, independent of $h$, and there exists $\boldsymbol{\psi}_{0} \in \mathbf{H}^{1 / 2}(\Sigma)$, such that

$$
\begin{gathered}
\sup _{\tau_{h} \in \mathbf{H}_{h}\left(\Omega_{\mathrm{S}}\right) \backslash \mathbf{0}} \frac{\left(\operatorname{div} \tau_{h}, v_{h}\right)_{\mathrm{S}}}{\left\|\tau_{h}\right\|_{\operatorname{div}, \Omega_{\mathrm{S}}}} \geq \beta_{\mathrm{S}}\left\|v_{h}\right\|_{0, \Omega_{\mathrm{S}}} \quad \forall v_{h} \in L_{h}\left(\Omega_{\mathrm{S}}\right), \\
\sup _{\mathbf{v}_{h} \in \mathbf{H}_{h}\left(\Omega_{\mathrm{D}}\right) \backslash \mathbf{0}} \frac{\left(\operatorname{div} \mathbf{v}_{h}, q_{h}\right)_{\mathrm{D}}}{\left\|\mathbf{v}_{h}\right\|_{\operatorname{div}, \Omega_{\mathrm{D}}}} \geq \beta_{\mathrm{D}}\left\|q_{h}\right\|_{0, \Omega_{\mathrm{D}}} \quad \forall q_{h} \in L_{h, 0}\left(\Omega_{\mathrm{D}}\right), \\
\boldsymbol{\psi}_{0} \in \boldsymbol{\Lambda}_{h}^{\mathrm{S}}(\Sigma) \quad \forall h \quad \text { and }\left\langle\boldsymbol{\psi}_{0} \cdot \mathbf{n}, 1\right\rangle_{\Sigma} \neq 0 .
\end{gathered}
$$

On the other hand, we now look at the discrete kernel of $\mathcal{B}$, which is defined by

$$
\mathbb{V}_{h}:=\left\{\underline{\boldsymbol{\tau}}_{h} \in \mathbb{X}_{h, 0}: \quad \mathcal{B}\left(\underline{\boldsymbol{\tau}}_{h}, \underline{\mathbf{v}}_{h}\right)=0 \quad \forall \underline{\mathbf{v}}_{h} \in \mathbb{M}_{h, 0}\right\}
$$

In order to have a more explicit definition of $\mathbb{V}_{h}$ we introduce the following assumption:

(H.2) $\operatorname{div} \mathbf{H}_{h}\left(\Omega_{\mathrm{S}}\right) \subseteq L_{h}\left(\Omega_{\mathrm{S}}\right) \quad$ and $\operatorname{div} \mathbf{H}_{h}\left(\Omega_{\mathrm{D}}\right) \subseteq L_{h}\left(\Omega_{\mathrm{D}}\right)$.

It follows from the definition of $\mathcal{B}($ cf. (2.30) $)$ and (H.2) that $\mathbb{V}_{h}=\mathbb{V}_{1, h} \times \mathbb{V}_{2, h}$, where

$$
\mathbb{V}_{1, h}=\tilde{\mathbb{H}}_{h, 0}\left(\Omega_{\mathrm{S}}\right) \times \tilde{\mathbf{H}}_{h}\left(\Omega_{\mathrm{D}}\right) \quad \text { and } \quad \mathbb{V}_{2, h}=\tilde{\Lambda}_{h}^{\mathrm{S}}(\Sigma) \times \Lambda_{h}^{\mathrm{D}}(\Sigma),
$$


with

$$
\begin{gathered}
\tilde{\mathbb{H}}_{h, 0}\left(\Omega_{\mathrm{S}}\right):=\left\{\boldsymbol{\tau}_{h} \in \mathbb{H}_{h, 0}\left(\Omega_{\mathrm{S}}\right): \quad \operatorname{div} \boldsymbol{\tau}_{h}=\mathbf{0}\right\} \\
\tilde{\mathbf{H}}_{h}\left(\Omega_{\mathrm{D}}\right):=\left\{\mathbf{v}_{h} \in \mathbf{H}_{h}\left(\Omega_{\mathrm{D}}\right): \quad \operatorname{div} \mathbf{v}_{h} \in \mathbb{P}_{0}\left(\Omega_{\mathrm{D}}\right)\right\}
\end{gathered}
$$

and

$$
\tilde{\Lambda}_{h}^{\mathrm{S}}(\Sigma):=\left\{\boldsymbol{\psi}_{h} \in \boldsymbol{\Lambda}_{h}^{\mathrm{S}}(\Sigma): \quad\left\langle\boldsymbol{\psi}_{h} \cdot \mathbf{n}, 1\right\rangle_{\Sigma}=0\right\} .
$$

Note that $\mathbb{V}_{h} \subseteq \mathbb{V}$, which yields, in particular, $\mathbb{V}_{1, h} \subseteq \mathbb{V}_{1}$.

Then, applying the same diagonal argument employed in the proof of Lemma 3.8. we find that $\mathbf{b}$ satisfies the discrete inf-sup condition uniformly on $\mathbb{V}_{1, h} \times \mathbb{V}_{2, h}$ if and only if there exist $\tilde{\beta}_{\mathrm{S}}, \tilde{\beta}_{\mathrm{D}}>0$, independent of $h$, such that

$$
\begin{aligned}
& \sup _{\boldsymbol{\tau}_{h} \in \tilde{\mathbb{H}}_{h, 0}\left(\Omega_{\mathrm{S}}\right) \backslash \mathbf{0}} \frac{\left\langle\boldsymbol{\tau}_{h} \mathbf{n}, \boldsymbol{\psi}_{h}\right\rangle_{\Sigma}}{\left\|\boldsymbol{\tau}_{h}\right\|_{\operatorname{div}, \Omega_{\mathrm{S}}}} \geq \tilde{\beta}_{\mathrm{S}}\left\|\boldsymbol{\psi}_{h}\right\|_{1 / 2, \Sigma} \quad \forall \boldsymbol{\psi}_{h} \in \tilde{\boldsymbol{\Lambda}}_{h}^{\mathrm{S}}(\Sigma), \\
& \sup _{\mathbf{v}_{h} \in \tilde{\mathbf{H}}_{h}\left(\Omega_{\mathrm{D}}\right) \backslash \mathbf{0}} \frac{\left\langle\mathbf{v}_{h} \cdot \mathbf{n}, \xi_{h}\right\rangle_{\Sigma}}{\left\|\mathbf{v}_{h}\right\|_{\text {div }, \Omega_{\mathrm{D}}}} \geq \tilde{\beta}_{\mathrm{D}}\left\|\xi_{h}\right\|_{1 / 2, \Sigma} \quad \forall \xi_{h} \in \Lambda_{h}^{\mathrm{D}}(\Sigma) .
\end{aligned}
$$

In addition, the characterization of the elements of $\tilde{\Lambda}_{h}^{\mathrm{S}}(\Sigma)$ yields the supremum in (4.15) to remain unchanged if taken on $\tilde{\mathbb{H}}_{h}\left(\Omega_{\mathrm{S}}\right):=\left\{\boldsymbol{\tau}_{h} \in \mathbb{H}_{h}\left(\Omega_{\mathrm{S}}\right): \operatorname{div} \boldsymbol{\tau}_{h}=\mathbf{0}\right\}$ instead of $\tilde{\mathbb{H}}_{h, 0}\left(\Omega_{\mathrm{S}}\right)$, and therefore it is not difficult to see that a sufficient condition for (4.15) is given by:

$$
\sup _{\tau_{h} \in \tilde{\mathbf{H}}_{h}\left(\Omega_{\mathrm{S}}\right) \backslash \mathbf{0}} \frac{\left\langle\tau_{h} \cdot \mathbf{n}, \psi_{h}\right\rangle_{\Sigma}}{\left\|\tau_{h}\right\|_{\mathrm{div}, \Omega_{\mathrm{S}}}} \geq \tilde{\beta}_{\mathrm{S}}\left\|\psi_{h}\right\|_{1 / 2, \Sigma} \quad \forall \psi_{h} \in \Lambda_{h}^{\mathrm{S}}(\Sigma),
$$

where

$$
\tilde{\mathbf{H}}_{h}\left(\Omega_{\mathrm{S}}\right):=\left\{\tau_{h} \in \mathbf{H}_{h}\left(\Omega_{\mathrm{S}}\right): \operatorname{div} \tau_{h}=0\right\} .
$$

In this way, we now add the following hypothesis:

(H.3) There exist $\tilde{\beta}_{\mathrm{S}}, \tilde{\beta}_{\mathrm{D}}>0$, independent of $h$, such that

$$
\begin{array}{cl}
\sup _{\tau_{h} \in \tilde{\mathbf{H}}_{h}\left(\Omega_{\mathrm{S}}\right) \backslash \mathbf{0}} \frac{\left\langle\tau_{h} \cdot \mathbf{n}, \psi_{h}\right\rangle_{\Sigma}}{\left\|\tau_{h}\right\|_{\operatorname{div}, \Omega_{\mathrm{S}}}} \geq \tilde{\beta}_{\mathrm{S}}\left\|\psi_{h}\right\|_{1 / 2, \Sigma} & \forall \psi_{h} \in \Lambda_{h}^{\mathrm{S}}(\Sigma), \\
\sup _{\mathbf{v}_{h} \in \tilde{\mathbf{H}}_{h}\left(\Omega_{\mathrm{D}}\right) \backslash \mathbf{0}} \frac{\left\langle\mathbf{v}_{h} \cdot \mathbf{n}, \xi_{h}\right\rangle_{\Sigma}}{\left\|\mathbf{v}_{h}\right\|_{\operatorname{div}, \Omega_{\mathrm{D}}}} \geq \tilde{\beta}_{\mathrm{D}}\left\|\xi_{h}\right\|_{1 / 2, \Sigma} \quad \forall \xi_{h} \in \Lambda_{h}^{\mathrm{D}}(\Sigma) .
\end{array}
$$

We end this section by mentioning that for computational purposes we replace the Galerkin scheme (4.7) by the equivalent one arising from the utilization of the decomposition (4.8). In other words, we drop the explicit unknown approximating $\mu \in \mathbb{R}$ and keep it implicitly by redefining the approximation of the pseudostress $\sigma_{\mathrm{S}} \in \mathbb{H}\left(\mathbf{d i v} ; \Omega_{\mathrm{S}}\right)$ as an unknown in $\mathbb{H}_{h}\left(\Omega_{\mathrm{S}}\right)$. This can also be seen as a discrete version of the reconstruction of $\boldsymbol{\sigma}_{\mathrm{S}}$ from the decomposition (2.21). In this way, the equivalent Galerkin scheme reduces to: Find $\left(\underline{\boldsymbol{\sigma}}_{h}, \underline{\mathbf{u}}_{h}\right) \in \mathbb{X}_{h} \times \mathbb{M}_{h}$ such that

$$
\begin{aligned}
\mathcal{A}\left(\underline{\boldsymbol{\sigma}}_{h}, \underline{\boldsymbol{\tau}}_{h}\right)+\mathcal{B}\left(\underline{\boldsymbol{\tau}}_{h}, \underline{\mathbf{u}}_{h}\right) & =\mathcal{F}\left(\underline{\boldsymbol{\tau}}_{h}\right) \quad \forall \underline{\boldsymbol{\tau}}_{h} \in \mathbb{X}_{h}, \\
\mathcal{B}\left(\underline{\boldsymbol{\sigma}}_{h}, \underline{\mathbf{v}}_{h}\right) & =\mathcal{G}\left(\underline{\mathbf{v}}_{h}\right) \quad \forall \underline{\mathbf{v}}_{h} \in \mathbb{M}_{h},
\end{aligned}
$$


where

$$
\begin{aligned}
\mathbb{X}_{h} & :=\mathbb{H}_{h}\left(\Omega_{\mathrm{S}}\right) \times \mathbf{H}_{h}\left(\Omega_{\mathrm{D}}\right) \times \boldsymbol{\Lambda}_{h}^{\mathrm{S}}(\Sigma) \times \Lambda_{h}^{\mathrm{D}}(\Sigma), \\
\mathbb{M}_{h} & :=\mathbf{L}_{h}\left(\Omega_{\mathrm{S}}\right) \times L_{h, 0}\left(\Omega_{\mathrm{D}}\right),
\end{aligned}
$$

and $\mathcal{B}$ is redefined by suppressing the third term on the right-hand side of (2.30). The numerical results shown below in Section [6 consider precisely this scheme in which the mean value condition of $L_{h, 0}\left(\Omega_{\mathrm{D}}\right)$ is imposed through a Lagrange multiplier.

4.2. The main result. The following theorem establishes the well posedness of (4.7) and the associated Cea estimate.

Theorem 4.1. Assume that the hypotheses (H.0), (H.1), (H.2), and (H.3) hold. Then the Galerkin scheme (4.7) has a unique solution $\left(\underline{\boldsymbol{\sigma}}_{h}, \underline{\mathbf{u}}_{h}\right) \in \mathbb{X}_{h, 0} \times \mathbb{M}_{h, 0}$ and there exists $C_{1}>0$, independent of $h$, such that

$$
\left\|\left(\underline{\boldsymbol{\sigma}}_{h}, \underline{\mathbf{u}}_{h}\right)\right\|_{\mathbb{X} \times \mathbb{M}} \leq C_{1}\left\{\left\|\left.\mathcal{F}\right|_{\mathbb{X}_{h, 0}}\right\|_{\mathbb{X}_{h, 0}^{\prime}}+\left\|\left.\mathcal{G}\right|_{\mathbb{M}_{h, 0}}\right\|_{\mathbb{M}_{h, 0}^{\prime}}\right\} .
$$

In addition, there exists $C_{2}>0$, independent of $h$, such that

$$
\left\|\underline{\boldsymbol{\sigma}}-\underline{\boldsymbol{\sigma}}_{h}\right\|_{\mathbb{X}}+\left\|\underline{\mathbf{u}}-\underline{\mathbf{u}}_{h}\right\|_{\mathbb{M}} \leq C_{2}\left\{\inf _{\underline{\boldsymbol{\tau}}_{h} \in \mathbb{X}_{h, 0}}\left\|\underline{\boldsymbol{\sigma}}-\underline{\boldsymbol{\tau}}_{h}\right\|_{\mathbb{X}}+\inf _{\underline{\mathbf{v}}_{h} \in \mathbb{M}_{h, 0}}\left\|\underline{\mathbf{u}}-\underline{\mathbf{v}}_{h}\right\|_{\mathbb{M}}\right\},
$$

where $(\underline{\boldsymbol{\sigma}}, \underline{\mathbf{u}}) \in \mathbb{X}_{0} \times \mathbb{M}_{0}$ is the unique solution of (3.2) .

Proof. It is clear from the analysis in Section 4.1 that (H.1) (resp. (H.3)) implies the discrete inf-sup condition for $\mathcal{B}$ (resp. for b) uniformly on $\mathbb{X}_{h, 0} \times \mathbb{M}_{h, 0}$ (resp. on $\left.\mathbb{V}_{1, h} \times \mathbb{V}_{2, h}\right)$. In addition, the fact that $\mathbb{V}_{1, h} \subseteq \mathbb{V}_{1}$ and Lemma 3.7 imply that a is uniformly elliptic in $\mathbb{V}_{1, h}$, whereas $\mathbf{c}$ is trivially positive semidefinite on $\mathbb{V}_{2, h} \subseteq$ $\mathbb{V}_{2} \subseteq \mathbf{H}^{1 / 2}(\Sigma) \times H^{1 / 2}(\Sigma)$ (cf. (3.7) $)$. In this way, applying the discrete version of Lemma 3.4 we conclude that the discrete operator induced by $\mathcal{A}$ is invertible in $\mathbb{V}_{h}$ with uniformly bounded inverse. Therefore, the rest of the proof reduces to a straightforward application of the discrete Babuška-Brezzi theory (see, e.g. [23, Theorem II.1.1], [8, Chapter II]).

It is important to remark here that the second and third terms defining the bilinear form $\mathbf{c}$ are the only ones in the whole variational system where the Darcy and Stokes discrete spaces meet. However, it is also clear from the previous proof that these terms do not play any role in the stability analysis of the Galerkin scheme since $\mathbf{c}$ is already positive semidefinite in the whole space $\mathbf{H}^{1 / 2}(\Sigma) \times H^{1 / 2}(\Sigma)$. This fact also explains why each one of the hypotheses (H.0), (H.1), (H.2), and (H.3), is formed by independent conditions concerning the subspaces for the Stokes and Darcy domains separately. Nevertheless, we notice that these independent assumptions show analogue structures, particularly with respect to the kind of operators and continuous spaces involved: compare for instance (4.12) with (4.13) in (H.1) and (4.17) with (4.18) in (H.3). This fact confirms the strong possibility of deriving stable finite element subspaces of the same kind in both domains. A specific example in this direction employing the well-known Raviart-Thomas subspaces is given precisely in Section 5 below.

Meanwhile, we prove next that the existence of uniformly bounded discrete liftings for the normal traces on $\Sigma$ coming from both regions simplifies the statement of (H.3). 
4.3. Stable discrete liftings. The aim of this section is to give sufficient conditions for the inf-sup inequalities (4.17) and (4.18) in hypothesis (H.3). These new conditions have to do with the eventual existence of stable discrete liftings of the normal traces on $\Sigma$, and they will be working hypotheses that can be more easily verified for each set of discrete spaces. In particular, these will be the conditions that we will verify for the example with Raviart-Thomas elements in Section 5 .

We notice first that conditions (4.17) and (4.18) are hypotheses that deal with how the normal components of elements of $\tilde{\mathbf{H}}_{h}\left(\Omega_{\mathrm{S}}\right)$ and $\tilde{\mathbf{H}}_{h}\left(\Omega_{\mathrm{D}}\right)$ are tested with $\Lambda_{h}^{\mathrm{S}}(\Sigma)$ and $\Lambda_{h}^{\mathrm{D}}(\Sigma)$, respectively. Because of the already mentioned analogue structure of these assumptions, we summarize them as follows with $\star \in\{\mathrm{S}, \mathrm{D}\}$ :

$$
\sup _{\mathbf{v}_{h} \in \tilde{\mathbf{H}}_{h}\left(\Omega_{\star}\right) \backslash \mathbf{0}} \frac{\left\langle\mathbf{v}_{h} \cdot \mathbf{n}, \xi_{h}\right\rangle_{\Sigma}}{\left\|\mathbf{v}_{h}\right\|_{\operatorname{div}, \Omega_{\star}}} \geq \tilde{\beta}_{\star}\left\|\xi_{h}\right\|_{1 / 2, \Sigma} \quad \forall \xi_{h} \in \Lambda_{h}^{\star}(\Sigma) .
$$

This kind of condition can be broken into two pieces. Let us recall from Section 4.1 that

$$
\begin{gathered}
\tilde{\mathbf{H}}_{h}\left(\Omega_{\mathrm{S}}\right):=\left\{\mathbf{v}_{h} \in \mathbf{H}_{h}\left(\Omega_{\mathrm{S}}\right): \quad \operatorname{div} \mathbf{v}_{h}=0\right\}, \\
\tilde{\mathbf{H}}_{h}\left(\Omega_{\mathrm{D}}\right):=\left\{\mathbf{v}_{h} \in \mathbf{H}_{h}\left(\Omega_{\mathrm{D}}\right): \operatorname{div} \mathbf{v}_{h} \in \mathbb{P}_{0}\left(\Omega_{\mathrm{D}}\right)\right\},
\end{gathered}
$$

and for $\star \in\{\mathrm{S}, \mathrm{D}\}$ define

$$
\Phi_{h}^{\star}(\Sigma):=\left\{\left.\mathbf{v}_{h} \cdot \mathbf{n}\right|_{\Sigma}: \quad \mathbf{v}_{h} \in \tilde{\mathbf{H}}_{h}\left(\Omega_{\star}\right)\right\} .
$$

Assume that the linear operator $\mathbf{v}_{h} \mapsto \mathbf{v}_{h} \cdot \mathbf{n}$ from $\tilde{\mathbf{H}}_{h}\left(\Omega_{\star}\right)$ to $\Phi_{h}^{\star}(\Sigma)$ has a uniformly bounded right inverse, i.e., there exist a linear operator $\mathcal{L}_{h}^{\star}: \Phi_{h}^{*}(\Sigma) \rightarrow$ $\tilde{\mathbf{H}}_{h}\left(\Omega_{\mathrm{S}}\right)$ and $c_{\star}>0$, independent of $h$, such that

$$
\begin{aligned}
& \left\|\mathcal{L}_{h}^{\star}\left(\phi_{h}\right)\right\|_{\operatorname{div}, \Omega_{\star}} \leq c_{\star}\left\|\phi_{h}\right\|_{-1 / 2, \Sigma} \text { and } \\
& \mathcal{L}_{h}^{\star}\left(\phi_{h}\right) \cdot \mathbf{n}=\phi_{h} \quad \text { on } \quad \Sigma \quad \forall \phi_{h} \in \Phi_{h}^{\star}(\Sigma) .
\end{aligned}
$$

Such a uniformly bounded right inverse of the normal trace will henceforth be referred to as a stable discrete lifting to $\Omega_{\star}(\star \in\{\mathrm{S}, \mathrm{D}\})$. Note that by [15], existence of $\mathcal{L}_{h}^{\star}$ satisfying (4.25) is equivalent to existence of a Scott-Zhang type operator $\boldsymbol{\pi}_{h}^{\star}: \mathbf{H}\left(\operatorname{div} ; \Omega_{\star}\right) \rightarrow \tilde{\mathbf{H}}_{h}\left(\Omega_{\star}\right)$, linear and uniformly bounded, such that

$$
\begin{gathered}
\boldsymbol{\pi}_{h}^{*}\left(\mathbf{v}_{h}\right)=\mathbf{v}_{h} \quad \forall \mathbf{v}_{h} \in \tilde{\mathbf{H}}_{h}\left(\Omega_{\star}\right), \quad \text { and } \quad \mathbf{v} \cdot \mathbf{n}=0 \text { on } \Sigma \\
\Longrightarrow\left(\boldsymbol{\pi}_{h}^{\star}(\mathbf{v})\right) \cdot \mathbf{n}=0 \quad \text { on } \Sigma .
\end{gathered}
$$

The following lemma reduces the inf-sup condition (4.22) to the inherited interaction between the elements of $\Phi_{h}^{\star}(\Sigma)$ and $\Lambda_{h}^{\star}(\Sigma)$.

Lemma 4.2. Assume that there exists a stable discrete lifting to $\Omega_{\star}$. Then (4.22) is equivalent to the existence of $\tilde{\beta}_{\star}>0$, independent of $h$, such that

$$
\sup _{\phi_{h} \in \Phi_{h}^{\star}(\Sigma) \backslash \mathbf{0}} \frac{\left\langle\phi_{h}, \xi_{h}\right\rangle_{\Sigma}}{\left\|\phi_{h}\right\|_{-1 / 2, \Sigma}} \geq \tilde{\beta}_{\star}\left\|\xi_{h}\right\|_{1 / 2, \Sigma} \quad \forall \xi_{h} \in \Lambda_{h}^{\star}(\Sigma) .
$$


Proof. It suffices to show that there exist $C_{1}, C_{2}>0$, independent of $h$, such that for each $\xi_{h} \in \Lambda_{h}^{\star}(\Sigma)$ there holds

$$
\begin{aligned}
C_{1} \sup _{\phi_{h} \in \Phi_{h}^{\star}(\Sigma) \backslash \mathbf{0}} \frac{\left\langle\phi_{h}, \xi_{h}\right\rangle_{\Sigma}}{\left\|\phi_{h}\right\|_{-1 / 2, \Sigma}} & \leq \sup _{\mathbf{v}_{h} \in \tilde{\mathbf{H}}_{h}\left(\Omega_{\star}\right) \backslash \mathbf{0}} \frac{\left\langle\mathbf{v}_{h} \cdot \mathbf{n}, \xi_{h}\right\rangle_{\Sigma}}{\left\|\mathbf{v}_{h}\right\|_{\text {div }, \Omega_{\star}}} \\
& \leq C_{2} \sup _{\phi_{h} \in \Phi_{h}^{\star}(\Sigma) \backslash \mathbf{0}} \frac{\left\langle\phi_{h}, \xi_{h}\right\rangle_{\Sigma}}{\left\|\phi_{h}\right\|_{-1 / 2, \Sigma}} .
\end{aligned}
$$

In fact, on the one hand,

$$
\frac{\left\langle\phi_{h}, \xi_{h}\right\rangle_{\Sigma}}{\left\|\phi_{h}\right\|_{-1 / 2, \Sigma}} \leq c_{\star} \frac{\left\langle\phi_{h}, \xi_{h}\right\rangle_{\Sigma}}{\left\|\mathcal{L}_{h}^{\star}\left(\phi_{h}\right)\right\|_{\operatorname{div}, \Omega_{\star}}} \leq c_{\star} \sup _{\mathbf{v}_{h} \in \tilde{\mathbf{H}}_{h}\left(\Omega_{\star}\right) \backslash \mathbf{0}} \frac{\left\langle\mathbf{v}_{h} \cdot \mathbf{n}, \xi_{h}\right\rangle_{\Sigma}}{\left\|\mathbf{v}_{h}\right\|_{\text {div }, \Omega_{\star}}} \quad \forall \phi_{h} \in \Phi_{h}^{\star}(\Sigma),
$$

and on the other hand,

$$
\frac{\left\langle\mathbf{v}_{h} \cdot \mathbf{n}, \xi_{h}\right\rangle_{\Sigma}}{\left\|\mathbf{v}_{h}\right\|_{\text {div }, \Omega_{\star}}} \leq C \frac{\left\langle\mathbf{v}_{h} \cdot \mathbf{n}, \xi_{h}\right\rangle_{\Sigma}}{\left\|\mathbf{v}_{h} \cdot \mathbf{n}\right\|_{-1 / 2, \Sigma}} \leq C \sup _{\phi_{h} \in \Phi_{h}^{\star}(\Sigma) \backslash \mathbf{0}} \frac{\left\langle\phi_{h}, \xi_{h}\right\rangle_{\Sigma}}{\left\|\phi_{h}\right\|_{-1 / 2, \Sigma}} \quad \forall \mathbf{v}_{h} \in \tilde{\mathbf{H}}_{h}\left(\Omega_{\star}\right),
$$

which yield (4.27) with $C_{1}=1 / c_{\star}$ and $C_{2}=C$.

We have thus proved that the existence of stable discrete liftings to $\Omega_{\mathrm{S}}$ and $\Omega_{\mathrm{D}}$ together with the inf-sup condition (4.26) constitute sufficient conditions for (H.3) to hold. In this respect, we find it important to emphasize that (4.26) deals exclusively with spaces of functions defined on the interface $\Sigma$.

\section{A particular Choice of Discrete spaCES}

5.1. Discretization of the domains. Let $\mathcal{T}_{h}^{\mathrm{S}}$ and $\mathcal{T}_{h}^{\mathrm{D}}$ be respective triangulations of the domains $\Omega_{\mathrm{S}}$ and $\Omega_{\mathrm{D}}$ formed by shape-regular triangles in the usual conditions of the finite element literature. Assume that these triangulations match in $\Sigma$, so that $\mathcal{T}_{h}^{\mathrm{S}} \cup \mathcal{T}_{h}^{\mathrm{D}}$ is a triangulation of $\Omega_{\mathrm{S}} \cup \Sigma \cup \Omega_{\mathrm{D}}$. Let $\Sigma_{h}$ be the partition of $\Sigma$ inherited from $\mathcal{T}_{h}^{\mathrm{S}}\left(\right.$ or $\mathcal{T}_{h}^{\mathrm{D}}$ ). Then, given a triangle $T$ we consider the local Raviart-Thomas space of the lowest order

$$
\mathrm{RT}_{0}(T):=\operatorname{span}\left\{(1,0),(0,1),\left(x_{1}, x_{2}\right)\right\} .
$$

We then define one Raviart-Thomas space on each subdomain and their usual companion spaces of piecewise constant functions: for $\star \in\{S, D\}$,

$$
\begin{aligned}
\mathbf{H}_{h}\left(\Omega_{\star}\right) & :=\left\{\mathbf{v}_{h} \in \mathbf{H}\left(\operatorname{div} ; \Omega_{\star}\right):\left.\quad \mathbf{v}_{h}\right|_{T} \in \mathrm{RT}_{0}(T) \quad \forall T \in \mathcal{T}_{h}^{\star}\right\}, \\
L_{h}\left(\Omega_{\star}\right) & :=\left\{q_{h}: \Omega_{\star} \rightarrow \mathbb{R}:\left.\quad q_{h}\right|_{T} \in \mathbb{P}_{0}(T) \quad \forall T \in \mathcal{T}_{h}^{\star}\right\} .
\end{aligned}
$$

It is clear that (H.0) and (H.2) are satisfied and it is well known that the discrete inf-sup conditions (4.12) and (4.13) in (H.1) are as well (see, e.g. 8, Chapter IV] or [31, Chapter 7]). Moreover, the spaces $\Phi_{h}^{\mathrm{S}}(\Sigma)$ and $\Phi_{h}^{\mathrm{D}}(\Sigma)$ of discrete normal traces on $\Sigma$ (cf. (4.24) ) are, for the time being, contained in

$$
\Phi_{h}(\Sigma):=\left\{\xi_{h}: \Sigma \rightarrow \mathbb{R}:\left.\quad \xi_{h}\right|_{e} \in \mathbb{P}_{0}(e) \quad \forall \text { edge } e \in \Sigma_{h}\right\} .
$$

We will see later on, as a corollary of Lemma 5.1 below, that actually $\Phi_{h}^{\mathrm{S}}(\Sigma)=$ $\Phi_{h}^{\mathrm{D}}(\Sigma)=\Phi_{h}(\Sigma)$. 
Now, although we could keep our options open for the remaining spaces $\Lambda_{h}^{\mathrm{S}}(\Sigma)$ and $\Lambda_{h}^{\mathrm{D}}(\Sigma)$, we simplify the situation by taking

$$
\Lambda_{h}^{\mathrm{S}}(\Sigma)=\Lambda_{h}^{\mathrm{D}}(\Sigma)=\Lambda_{h}(\Sigma) .
$$

Gathering Theorem 4.1 and Lemma 4.2 we are left with the following tasks:

i) Prove the existence of stable discrete liftings (or give conditions on the grid that ensure their existence).

ii) Choose $\Lambda_{h}(\Sigma)$ such that we can find $\boldsymbol{\psi}_{0} \in \mathbf{H}^{1 / 2}(\Sigma)$ satisfying $\boldsymbol{\psi}_{0} \in \boldsymbol{\Lambda}_{h}(\Sigma)$ $\forall h$ and $\left\langle\boldsymbol{\psi}_{0} \cdot \mathbf{n}, 1\right\rangle_{\Sigma} \neq 0$ (cf. (4.14) in (H.1)), and such that the inf-sup condition (4.26) holds.

In Sections 5.2 and 5.3 below we deal precisely with i) and ii), respectively.

5.2. The discrete liftings. We are going to be able to construct discrete liftings to $\Omega_{\mathrm{S}}$ and $\Omega_{\mathrm{D}}$ by demanding some additional conditions on the triangulations. Namely, we ask for $\mathcal{T}_{h}^{\mathrm{S}}$ and $\mathcal{T}_{h}^{\mathrm{D}}$ to be quasiuniform in a neighborhood of $\Sigma$. More precisely, we assume that there is an open neighborhood of $\Sigma$, say $\Omega_{\Sigma}$, with Lipschitz boundary, and such that the elements intersecting that region are roughly of the same size. In other words, for $\star \in\{\mathrm{S}, \mathrm{D}\}$ we let $\Omega_{\star, \Sigma}:=\Omega_{\star} \cap \Omega_{\Sigma}$, define

$$
\mathcal{T}_{h, \Sigma}^{\star}:=\left\{T \in \mathcal{T}_{h}^{\star}: \quad T \cap \Omega_{\star, \Sigma} \neq \emptyset\right\}, \quad \mathcal{T}_{h, \Sigma}:=\mathcal{T}_{h, \Sigma}^{\mathrm{S}} \cup \mathcal{T}_{h, \Sigma}^{\mathrm{D}},
$$

and assume that there exists $c>0$, independent of $h$, such that

$$
\max _{T \in \mathcal{T}_{h, \Sigma}} h_{T} \leq c \min _{T \in \mathcal{T}_{h, \Sigma}} h_{T} .
$$

Because of the shape-regularity property, this implies that $\Sigma_{h}$ is quasiuniform, which means that there exists $C>0$, independent of $h$, such that

$$
h_{\Sigma}:=\max \left\{|e|: \quad e \in \Sigma_{h}\right\} \leq C \min \left\{|e|: \quad e \in \Sigma_{h}\right\} .
$$

Moreover, the quasiuniformity of $\Sigma_{h}$ implies the inverse inequality in $\Phi_{h}(\Sigma)$, that is,

(5.3) $\left\|\phi_{h}\right\|_{-1 / 2+\delta, \Sigma} \leq C h_{\Sigma}^{-\delta}\left\|\phi_{h}\right\|_{-1 / 2, \Sigma} \quad \forall \phi_{h} \in \Phi_{h}(\Sigma), \quad \forall \delta \in[0,1 / 2]$.

Next, in order to define the discrete liftings we need to introduce the RaviartThomas interpolation operator. For the forthcoming definitions and arguments $\star$ is a mute symbol taken in $\{\mathrm{S}, \mathrm{D}\}$. Hence, given a sufficiently smooth vector field $\mathbf{v}: \Omega_{\star} \rightarrow \mathbb{R}^{2}$, we define $\Pi_{h}^{\star}(\mathbf{v})$ as the only element of $\mathbf{H}_{h}\left(\Omega_{\star}\right)$ such that

$$
\int_{e} \Pi_{h}^{\star}(\mathbf{v}) \cdot \mathbf{n}=\int_{e} \mathbf{v} \cdot \mathbf{n} \quad \forall e \in \mathcal{E}_{h}^{\star},
$$

where $\mathcal{E}_{h}^{\star}$ is the set of the edges of the triangulation $\mathcal{T}_{h}^{\star}$. Let us review some properties of this operator that we will use in the sequel:

a) The interpolation operator $\Pi_{h}^{\star}$ is well defined in $\mathbf{H}^{\delta}\left(\Omega_{\star}\right) \cap \mathbf{H}\left(\operatorname{div} ; \Omega_{\star}\right)$ for any $\delta>0$ (see, e.g. [1, Theorem 3.1]).

b) For all $\mathbf{v}$ there holds $\operatorname{div} \Pi_{h}^{\star}(\mathbf{v})=\mathcal{P}_{h}^{\star}(\operatorname{div} \mathbf{v})$, where $\mathcal{P}_{h}^{\star}: L^{2}\left(\Omega_{\star}\right) \rightarrow$ $L_{h}\left(\Omega_{\star}\right)$ is the orthogonal projector. Equivalently,

$$
\left(\operatorname{div} \Pi_{h}^{\star}(\mathbf{v}), q_{h}\right)_{\star}=\left(\operatorname{div} \mathbf{v}, q_{h}\right)_{\star} \quad \forall q_{h} \in L_{h}\left(\Omega_{\star}\right) .
$$

This property is a simple consequence of the divergence theorem and the interpolation property (5.4) defining $\Pi_{h}^{\star}$. In particular, if $\operatorname{div} \mathbf{v} \equiv c$, it follows that $\operatorname{div} \Pi_{h}^{\star}(\mathbf{v}) \equiv c$. 
c) If $\mathbf{v} \cdot \mathbf{n} \in \Phi_{h}(\Sigma)$, then $\Pi_{h}^{\star}(\mathbf{v}) \cdot \mathbf{n}=\mathbf{v} \cdot \mathbf{n}$. This property also follows from (5.4).

d) There exists $C>0$, independent of $h$, such that for each $\mathbf{v} \in \mathbf{H}^{\delta}\left(\Omega_{\star}\right) \cap$ $\mathbf{H}\left(\operatorname{div} ; \Omega_{\star}\right)$, with $\delta \in(0,1]$, and for all $T \in \mathcal{T}_{h}^{\star}$, there holds (see, e.g. [25, Theorem 3.16])

$$
\left\|\mathbf{v}-\Pi_{h}^{*}(\mathbf{v})\right\|_{0, T} \leq C h_{T}^{\delta}\left\{|\mathbf{v}|_{\delta, T}+\|\operatorname{div} \mathbf{v}\|_{0, T}\right\}
$$

We are now in a position to establish the existence of stable discrete liftings.

Lemma 5.1. Assume that $\mathcal{T}_{h}^{\mathrm{S}}$ and $\mathcal{T}_{h}^{\mathrm{D}}$ are quasiuniform in a neighborhood $\Omega_{\Sigma}$ of $\Sigma$ as explained in the present section. Then there exist uniformly bounded linear operators $\mathcal{L}_{h}^{\star}: \Phi_{h}(\Sigma) \rightarrow \tilde{\mathbf{H}}_{h}\left(\Omega_{\star}\right)($ cf. (4.23) $)$ such that $\mathcal{L}_{h}^{\star}\left(\phi_{h}\right) \cdot \mathbf{n}=\phi_{h}$ on $\Sigma$ for each $\phi_{h} \in \Phi_{h}(\Sigma)$.

Proof. We start with the lifting to the Stokes domain $\Omega_{\mathrm{S}}$. First, we increase this region across the external boundary $\Gamma_{\mathrm{S}}$ to a new domain $\Omega_{\mathrm{S}}^{\text {ext }}$ with Lipschitz boundary $\Sigma \cup \Gamma_{\mathrm{S}}^{\text {ext }}$. Then we recall that $\Omega_{\mathrm{S}, \Sigma}:=\Omega_{\mathrm{S}} \cap \Omega_{\Sigma}$ and remark that $\Omega_{\mathrm{S}} \backslash \Omega_{\mathrm{S}, \Sigma}$ is interior to $\Omega_{\mathrm{S}}^{\mathrm{ext}}$, since both parts of its boundary lie at a nonzero distance of $\partial \Omega_{\mathrm{S}}^{\text {ext }}$. We refer to Figure 2 for the geometry. The thick lines enclose the extended Stokes domain $\Omega_{\mathrm{S}}^{\mathrm{ext}}$, whereas the shaded area corresponds to the neighborhood $\Omega_{\Sigma}$.

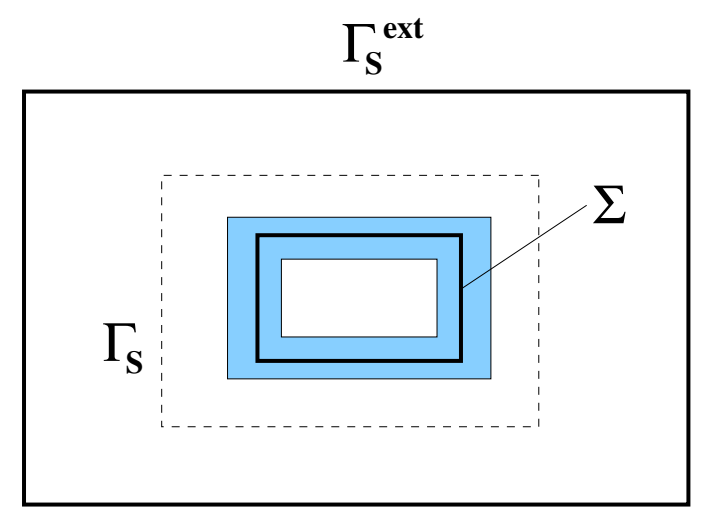

FiguRE 2. The domains in the proof of Lemma 5.1

We now begin the construction of our operator. Given $\phi_{h} \in \Phi_{h}(\Sigma)$, we let $v \in H^{1}\left(\Omega_{\mathrm{S}}^{\mathrm{ext}}\right)$ be the unique solution of the boundary value problem

$$
\Delta v=0 \quad \text { in } \Omega_{\mathrm{S}}^{\mathrm{ext}}, \quad v=0 \text { on } \Gamma_{\mathrm{S}}^{\mathrm{ext}}, \partial_{\mathbf{n}} v=\phi_{h} \quad \text { on } \quad \Sigma,
$$

which can be seen as a discrete version of (3.11). Then, as a consequence of the Lax-Milgram lemma and the classical regularity result on polygonal domains (see, e.g. [24]), we obtain, respectively, the following continuity bounds (we write them in the domains where they will be used):

$$
\begin{aligned}
\|v\|_{1, \Omega_{\mathrm{S}}} & \leq C_{1}\left\|\phi_{h}\right\|_{-1 / 2, \Sigma} \\
\|v\|_{5 / 4, \Omega_{\mathrm{S}}} & \leq C_{2}\left\|\phi_{h}\right\|_{-1 / 4, \Sigma}
\end{aligned}
$$


In addition, since $\Omega_{\mathrm{S}} \backslash \Omega_{\mathrm{S}, \Sigma}$ is an interior region of $\Omega_{\mathrm{S}}^{\text {ext }}$, the interior elliptic regularity estimate (see, e.g. [30, Theorem 4.16]) yields

$$
\|v\|_{2, \Omega_{\mathrm{S}} \backslash \Omega_{\mathrm{S}, \Sigma}} \leq C_{3}\left\|\phi_{h}\right\|_{-1 / 2, \Sigma},
$$

Note that, in particular, $\nabla v \in \mathbf{H}^{1 / 4}\left(\Omega_{\mathrm{S}}\right) \cap \mathbf{H}\left(\operatorname{div} ; \Omega_{\mathrm{S}}\right)$, and hence, thanks to a), we can define

$$
\mathcal{L}_{h}^{\mathrm{S}}\left(\phi_{h}\right):=\Pi_{h}^{\mathrm{S}}(\nabla v) \in \mathbf{H}_{h}\left(\Omega_{\mathrm{S}}\right) .
$$

Since $\operatorname{div} \nabla v=\Delta v=0 \quad$ in $\quad \Omega_{\mathrm{S}}$ and $\nabla v \cdot \mathbf{n}=\partial_{\mathbf{n}} v=\phi_{h} \in \Phi_{h}(\Sigma) \quad$ on $\quad \Sigma$, we deduce from $\mathbf{b}$ ) and $\mathbf{c}$ ), respectively, that

$$
\operatorname{div} \mathcal{L}_{h}^{S}\left(\phi_{h}\right)=0 \quad \text { in } \quad \Omega_{\mathrm{S}} \quad \text { and } \quad \mathcal{L}_{h}^{\mathrm{S}}\left(\phi_{h}\right) \cdot \mathbf{n}=\phi_{h} \quad \text { on } \quad \Sigma,
$$

which proves that $\mathcal{L}_{h}^{\mathrm{S}}$ is a lifting satisfying $\mathcal{L}_{h}^{\mathrm{S}}\left(\phi_{h}\right) \in \tilde{\mathbf{H}}_{h}\left(\Omega_{\mathrm{S}}\right) \quad \forall \phi_{h} \in \Phi_{h}(\Sigma)$.

It remains to show that $\mathcal{L}_{h}^{\mathrm{S}}$ is uniformly bounded. To this end, we divide $\Omega_{\mathrm{S}}$ into two regions

$$
\Omega_{\mathrm{S}, h}^{1}:=\bigcup\left\{T \in \mathcal{T}_{h}^{\mathrm{S}}: \quad T \notin \mathcal{T}_{h, \Sigma}^{\mathrm{S}}\right\} \subseteq \Omega_{\mathrm{S}} \backslash \Omega_{\mathrm{S}, \Sigma} \quad \text { and } \quad \Omega_{\mathrm{S}, h}^{2}:=\Omega_{\mathrm{S}} \backslash \Omega_{\mathrm{S}, h}^{1},
$$

where we recall that $\mathcal{T}_{h, \Sigma}^{\mathrm{S}}:=\left\{T \in \mathcal{T}_{h}^{\mathrm{S}}: \quad T \cap \Omega_{\mathrm{S}, \Sigma} \neq \emptyset\right\}$. Then, using (5.6), (5.8), and the stability of the Raviart-Thomas projection when applied to functions in $\mathbf{H}^{1}\left(\Omega_{\mathrm{S}, h}^{1}\right)$, we can bound:

$$
\begin{aligned}
\left\|\mathcal{L}_{h}^{\mathrm{S}}\left(\phi_{h}\right)\right\|_{\mathrm{div}, \Omega_{\mathrm{S}}} & =\left\|\mathcal{L}_{h}^{\mathrm{S}}\left(\phi_{h}\right)\right\|_{0, \Omega_{\mathrm{S}}} \leq\left\|\mathcal{L}_{h}^{\mathrm{S}}\left(\phi_{h}\right)\right\|_{0, \Omega_{\mathrm{S}, h}^{1}}+\left\|\mathcal{L}_{h}^{\mathrm{S}}\left(\phi_{h}\right)\right\|_{0, \Omega_{\mathrm{S}, h}^{2}} \\
& \leq\left\|\Pi_{h}^{\mathrm{S}}(\nabla v)\right\|_{0, \Omega_{\mathrm{S}, h}^{1}}+\|\nabla v\|_{0, \Omega_{\mathrm{S}, h}^{2}}+\left\|\nabla v-\Pi_{h}^{\mathrm{S}}(\nabla v)\right\|_{0, \Omega_{\mathrm{S}, h}^{2}} \\
& \leq C\left\{\|\nabla v\|_{1, \Omega_{\mathrm{S}} \backslash \Omega_{\mathrm{S}, \Sigma}}+\left\|\phi_{h}\right\|_{-1 / 2, \Sigma}+\left\|\nabla v-\Pi_{h}^{\mathrm{S}}(\nabla v)\right\|_{0, \Omega_{\mathrm{S}, h}^{2}}\right\} \\
& \leq C\left\{\left\|\phi_{h}\right\|_{-1 / 2, \Sigma}+\left\|\nabla v-\Pi_{h}^{\mathrm{S}}(\nabla v)\right\|_{0, \Omega_{\mathrm{S}, h}^{2}}\right\} .
\end{aligned}
$$

At the same time, applying (5.5) in $\mathbf{d})$ to $\nabla v \in \mathbf{H}^{1 / 4}\left(\Omega_{\mathrm{S}}\right) \cap \mathbf{H}\left(\operatorname{div} ; \Omega_{\mathrm{S}}\right)$, and employing the bound (5.7) and the inverse inequality (5.3) with $\delta=1 / 4$, we find that

$$
\begin{aligned}
\left\|\nabla v-\Pi_{h}^{\mathrm{S}}(\nabla v)\right\|_{0, \Omega_{\mathrm{S}, h}^{2}}^{2} & \leq C \sum_{T \in \mathcal{T}_{h, \Sigma}^{\mathrm{S}}} h_{T}^{1 / 2}\|\nabla v\|_{1 / 4, T}^{2} \leq C h_{\Sigma}^{1 / 2}\|v\|_{5 / 4, \Omega_{\mathrm{S}}}^{2} \\
& \leq C h_{\Sigma}^{1 / 2}\left\|\phi_{h}\right\|_{-1 / 4, \Sigma}^{2} \leq C\left\|\phi_{h}\right\|_{-1 / 2, \Sigma}^{2} .
\end{aligned}
$$

This estimate and the preceeding inequality give the result.

On the other hand, in the case of the Darcy domain $\Omega_{\mathrm{D}}$, the interface $\Sigma$ constitutes the whole boundary, which implies that $\Omega_{\mathrm{D}} \backslash \Omega_{\mathrm{D}, \Sigma}$ is interior to $\Omega_{\mathrm{D}}$, and hence there is no need to extend the domain to deal with regularity issues in the (nonexistent) remaining part of the boundary. According to this, given $\phi_{h} \in \Phi_{h}(\Sigma)$, we now define

$$
\mathcal{L}_{h}^{\mathrm{D}}\left(\phi_{h}\right):=\Pi_{h}^{\mathrm{D}}(\nabla v) \in \mathbf{H}_{h}\left(\Omega_{\mathrm{D}}\right),
$$

where $v \in H^{1}\left(\Omega_{\mathrm{D}}\right)$ is the unique solution of the bounday value problem

$$
\Delta v=\frac{1}{\left|\Omega_{\mathrm{D}}\right|} \int_{\Sigma} \phi_{h} \quad \text { in } \quad \Omega_{\mathrm{D}}, \quad \partial_{\mathbf{n}} v=\phi_{h} \quad \text { on } \quad \Sigma, \quad \int_{\Omega_{\mathrm{D}}} v=0,
$$

which can be seen as a discrete version of (3.12). Since

$\operatorname{div} \nabla v=\Delta v=\frac{1}{\left|\Omega_{\mathrm{D}}\right|} \int_{\Sigma} \phi_{h} \quad$ in $\quad \Omega_{\mathrm{D}} \quad$ and $\quad \nabla v \cdot \mathbf{n}=\partial_{\mathbf{n}} v=\phi_{h} \in \Phi_{h}(\Sigma) \quad$ on $\quad \Sigma$, 
we use again b) and c) to deduce, respectively, that

$$
\operatorname{div} \mathcal{L}_{h}^{\mathrm{D}}\left(\phi_{h}\right)=\frac{1}{\left|\Omega_{\mathrm{D}}\right|} \int_{\Sigma} \phi_{h} \in \mathbb{R} \quad \text { in } \quad \Omega_{\mathrm{S}} \quad \text { and } \quad \mathcal{L}_{h}^{\mathrm{D}}\left(\phi_{h}\right) \cdot \mathbf{n}=\phi_{h} \quad \text { on } \quad \Sigma,
$$

which proves that $\mathcal{L}_{h}^{\mathrm{D}}$ is a lifting satisfying $\mathcal{L}_{h}^{\mathrm{D}}\left(\phi_{h}\right) \in \tilde{\mathbf{H}}_{h}\left(\Omega_{\mathrm{D}}\right) \quad \forall \phi_{h} \in \Phi_{h}(\Sigma)$. The uniform boundedness of $\mathcal{L}_{h}^{\mathrm{D}}$ proceeds as in the previous case. We omit further details.

As a consequence of this lemma, and as already announced in Section 5.1, we now notice that $\Phi_{h}^{\mathrm{S}}(\Sigma)$ and $\Phi_{h}^{\mathrm{D}}(\Sigma)$ coincide with $\Phi_{h}(\Sigma)$ (cf. (5.2) ), and therefore the inf-sup condition (4.26) reduces simply to the existence of $\tilde{\beta}>0$, independent of $h$, such that

$$
\sup _{\phi_{h} \in \Phi_{h}(\Sigma) \backslash \mathbf{0}} \frac{\left\langle\phi_{h}, \xi_{h}\right\rangle_{\Sigma}}{\left\|\phi_{h}\right\|_{-1 / 2, \Sigma}} \geq \tilde{\beta}\left\|\xi_{h}\right\|_{1 / 2, \Sigma} \quad \forall \xi_{h} \in \Lambda_{h}(\Sigma) .
$$

5.3. Discretization on the interface. In this section we discuss how to choose $\Lambda_{h}(\Sigma)$ so that ii) is satisfied. In fact, there are many possible choices of $\Lambda_{h}(\Sigma)$ such that (5.9) holds, while the condition requiring the existence of $\boldsymbol{\psi}_{0} \in \mathbf{H}^{1 / 2}(\Sigma)$ such that $\boldsymbol{\psi}_{0} \in \Lambda_{h}(\Sigma) \forall h$ and $\left\langle\boldsymbol{\psi}_{0} \cdot \mathbf{n}, 1\right\rangle_{\Sigma} \neq 0$, is easy to verify if the sequence of subspaces is nested or if we are able to find a coarser space where the hypotheses hold.

OPTION 1. If the partition $\Sigma_{h}$ inherited from the interior triangulations is uniform, which is feasible only on very simple geometries $\Sigma$, we can take $\Lambda_{h}(\Sigma)$ to be the space of continuous linear elements of the dual grid, that is, on the grid whose nodes are the midpoints of $\Sigma_{h}$. Note that $\operatorname{dim} \Lambda_{h}(\Sigma)=\operatorname{dim} \Phi_{h}(\Sigma)$, and that on each corner of $\Sigma$ there is an element of the dual grid with half of its length on each of the edges that meet in that corner. The inf-sup condition (5.9) for these spaces is verified in [32, Lemma 6.4].

Option 2. Let $\widetilde{\Sigma}_{h}$ be another partition of $\Sigma$, completely independent from $\Sigma_{h}$, and then take

$$
\Lambda_{h}(\Sigma):=\mathbb{P}_{1}\left(\widetilde{\Sigma}_{h}\right) \cap \mathcal{C}(\Sigma), \quad \text { with } \quad \mathbb{P}_{1}\left(\widetilde{\Sigma}_{h}\right):=\prod_{e \in \widetilde{\Sigma}_{h}} \mathbb{P}_{1}(e)
$$

If both $\Sigma_{h}$ and $\widetilde{\Sigma}_{h}$ are quasiuniform, then there exists a constant $C_{0} \in(0,1]$ such that whenever

$$
h_{\Sigma} \leq C_{0} \widetilde{h}_{\Sigma}, \quad \widetilde{h}_{\Sigma}:=\max \left\{|\widetilde{e}|: \quad \widetilde{e} \in \widetilde{\Sigma}_{h}\right\},
$$

then (5.9) holds [5, Lemma 3.3]. In this case, if we assume that elements of $\widetilde{\Sigma}_{h}$ are segments (no element crosses a corner point), then $\boldsymbol{\psi}_{0}$ can be constructed exactly as explained at the end of the proof of Lemma 3.6.

Option 3. A very flexible (from the geometric point of view) construction of $\Lambda_{h}(\Sigma)$ can be done using a coarsened grid. Let us first assume that the number of edges of $\Sigma_{h}$ is an even number (we will show a simple strategy in case this number is odd at the end). Then, we let $\Sigma_{2 h}$ be the partition of $\Sigma$ arising by joining pairs of adjacent elements and define

$$
\Lambda_{h}(\Sigma):=\mathbb{P}_{1}\left(\Sigma_{2 h}\right) \cap \mathcal{C}(\Sigma) .
$$


Note that because $\Sigma_{h}$ is inherited from the interior triangulation, it is automatically of bounded variation (that is, the ratio of lengths of adjacent elements is bounded) and, therefore, so is $\Sigma_{2 h}$.

Lemma 5.2. The inf-sup condition (5.9) holds for the space (5.10).

Proof. We will actually prove an inequality that is more demanding than (5.9) (see (5.12) below). The structure of the proof (but not the result itself) follows closely [32, Section 7]. Let $\Sigma_{2 h}=\left\{e_{i} \mid i=1, \ldots, N\right\}$ be a numbering of the elements of the coarsened grid, where adjacent elements are numbered consecutively and where, in case of need, $e_{0}=e_{N}$ and $e_{1}=e_{N+1}$. Also, let $h_{i}:=\left|e_{i}\right|$. To each pair $\left(e_{i}, e_{i+1}\right)$ we assign a hat function $\eta_{i} \in \Lambda_{h}(\Sigma)$, supported in this pair and equal to one in the interior node $\bar{e}_{i} \cap \overline{e_{i+1}}$. Note that $\left\{\eta_{1}, \eta_{2}, \ldots, \eta_{N}\right\}$ is the usual basis of $\Lambda_{h}(\Sigma)$.

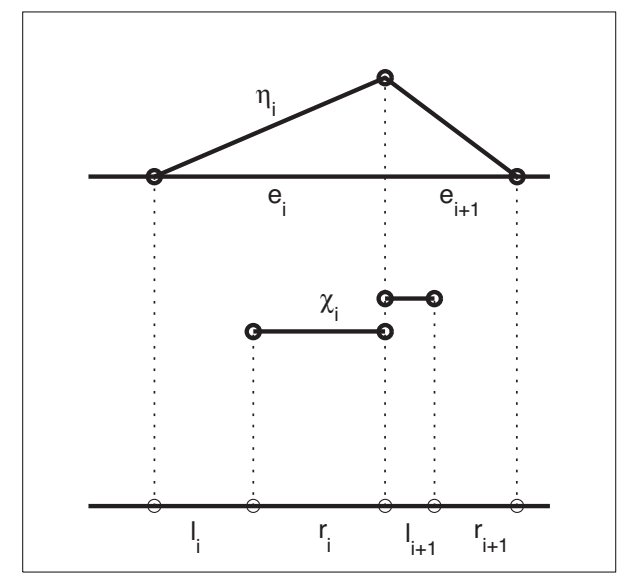

Figure 3. Construction of the basis functions of $\Phi_{h}^{\circ}$.

For each $e_{i} \in \Sigma_{2 h}$ there are two elements $l_{i}, r_{i} \in \Sigma_{h}$, whose union is $e_{i}$. They are tagged as left and right in the numbering direction of $\Sigma_{2 h}$, so that $r_{i}$ is adjacent to $l_{i+1}$ (see Figure 3). As a consequence of the bounded variation property

$$
0<C_{1} \leq c_{i}:=\frac{\left|r_{i}\right|}{h_{i}} \leq C_{2}<1 \quad \text { and } \quad 0<C_{3} \leq \frac{h_{i}}{h_{i+1}} \leq C_{4} \quad \forall i .
$$

We now define the piecewise constant function $\chi_{i} \in \Phi_{h}(\Sigma)$ given by

$$
\chi_{i}= \begin{cases}c_{i}^{-1} & \text { in } r_{i}, \\ \left(1-c_{i+1}\right)^{-1} & \text { in } l_{i+1}, \\ 0 & \text { otherwise. }\end{cases}
$$

The functions $\chi_{i}$ are mutually orthogonal in $L^{2}(\Sigma)$. We define

$$
\Phi_{h}^{\circ}:=\operatorname{span}\left\{\chi_{1}, \ldots, \chi_{N}\right\} \subset \Phi_{h}(\Sigma) .
$$


The aim of what follows is showing that there exists $C$ (which depends only on the four constants in (5.11) ) such that

$$
\sup _{\phi_{h} \in \Phi_{h}^{\circ} \backslash \mathbf{0}} \frac{\left\langle\xi_{h}, \phi_{h}\right\rangle_{\Sigma}}{\left\|\phi_{h}\right\|_{-s, \Sigma}} \geq C\left\|\xi_{h}\right\|_{s, \Sigma} \quad \forall \xi \in \Lambda_{h}(\Sigma), \quad s \in[0,1] .
$$

We will prove the result for $s=0$ and $s=1$. Given the fact that the dimensions of $\Lambda_{h}(\Sigma)$ and $\Phi_{h}^{\circ}$ coincide, an interpolation argument proves the result for the remaining cases. The case $s=1 / 2$ implies (5.9), since the supremum in this last inequality is taken over a larger space.

1st step. We first prove (5.12) for $s=0$. Here we follow [32, Proposition 7.1]. Let us define the operator $T_{h}: \Lambda_{h}(\Sigma) \rightarrow \Phi_{h}^{\circ}$ by

$$
T_{h} \xi_{h}=T_{h}\left(\sum_{i=1}^{N} \xi_{i} \eta_{i}\right):=\sum_{i=1}^{N} \xi_{i} \chi_{i} .
$$

Simple computations can be used to show that for all $\xi_{h} \in \Lambda_{h}(\Sigma)$,

$$
\begin{aligned}
\left\|\xi_{h}\right\|_{0, \Sigma}^{2} & \leq \frac{1}{2} \sum_{i=1}^{N} \xi_{i}^{2}\left(h_{i}+h_{i+1}\right), \\
\left\|T_{h} \xi_{h}\right\|_{0, \Sigma}^{2} & =\sum_{i=1}^{N} \xi_{i}^{2}\left(c_{i}^{-1} h_{i}+\left(1-c_{i+1}\right)^{-1} h_{i+1}\right) \leq C \sum_{i=1}^{N} \xi_{i}^{2}\left(h_{i}+h_{i+1}\right) \\
\left\langle\xi_{h}, T_{h} \xi_{h}\right\rangle_{\Sigma} & \geq \frac{1}{2} \sum_{i=1}^{N} \xi_{i}^{2}\left(h_{i}\left(\frac{3}{2}-c_{i}\right)+h_{i+1}\left(\frac{1}{2}+c_{i+1}\right)\right) \geq C \sum_{i=1}^{N} \xi_{i}^{2}\left(h_{i}+h_{i+1}\right) .
\end{aligned}
$$

These three inequalities can be used to prove (5.12) when $s=0$. Note that only the constants $C_{1}$ and $C_{2}$ of (5.11) are involved in these bounds.

2nd step. An intermediate step requires proving the following inequality:

$$
\sum_{i=1}^{N} h_{i}^{2} \int_{e_{i}}\left|\phi_{h}\right|^{2} \leq C \sum_{i=1}^{N}\left(\frac{\left\langle\phi_{h}, \eta_{i}\right\rangle_{\Sigma}}{\left\|\eta_{i}\right\|_{1, \Sigma}}\right)^{2} \quad \forall \phi_{h} \in \Phi_{h}^{\circ}
$$

The proof retraces the steps of [32, Lemma 7.2]. For integrals of $\Sigma$ we can use the arc parameterization $\mathbf{x}:[0,|\Sigma|] \rightarrow \Sigma$, where $|\Sigma|$ is the length of $\Sigma$, and identify

$$
\|\eta\|_{1, \Sigma}^{2}:=\int_{0}^{|\Sigma|}\left(|(\eta \circ \mathbf{x})(t)|^{2}+\left|(\eta \circ \mathbf{x})^{\prime}(t)\right|^{2}\right) \mathrm{d} t .
$$

Each of the following inequalities, valid for each $\eta_{i}$ and for arbitrary $\phi_{h}=\sum_{i=1}^{N} \phi_{i} \chi_{i}$ $\in \Phi_{h}^{\circ}$, is easy to prove:

$$
\begin{aligned}
\left\|\eta_{i}\right\|_{1, \Sigma}^{2} & =\frac{1}{3}\left(h_{i}+h_{i+1}\right)+h_{i}^{-1}+h_{i+1}^{-1} \leq C h_{i}^{-1}, \\
\sum_{i=1}^{N} h_{i}^{2} \int_{e_{i}}\left|\phi_{h}\right|^{2} & =\sum_{i=1}^{N} h_{i}^{3}\left(c_{i}^{-1} \phi_{i}^{2}+\left(1-c_{i}\right)^{-1} \phi_{i-1}^{2}\right) \leq C \sum_{i=1}^{N} \phi_{i}^{2} h_{i}^{3}, \\
\sum_{i=1}^{N} \phi_{i}^{2} h_{i}^{3} & \leq C \sum_{i=1}^{N}\left\langle\phi_{h}, \eta_{i}\right\rangle_{\Sigma} h_{i}^{2} \phi_{i} \\
& \leq C\left(\sum_{i=1}^{N}\left(\frac{\left\langle\phi_{h}, \eta_{i}\right\rangle_{\Sigma}}{\left\|\eta_{i}\right\|_{1, \Sigma}}\right)^{2}\right)^{1 / 2}\left(\sum_{i=1}^{N}\left\|\eta_{i}\right\|_{1, \Sigma}^{2} h_{i}^{4} \phi_{i}^{2}\right)^{1 / 2} .
\end{aligned}
$$


In particular, note that the second estimate uses that $\left|r_{i}\right|=c_{i} h_{i}$ and $\left|l_{i}\right|=\left(1-c_{i}\right) h_{i}$. From these inequalities the result follows readily.

3rd step. Once (5.13) has been proved, inequality (5.12) for $s=1$ can be proved following step by step the proof of [32, Proposition 7.3]. This finishes the proof of the lemma.

If the number of elements in $\Sigma_{h}$ is odd we simply reduce it to the even case. Indeed, in this case we can prove (5.9) for the subspace of $\Phi_{h}(\Sigma)$ consisting of elements such that the value of $\phi_{h}$ in a fixed set of two adjacent elements coincides. This fixed double element is considered as a single element and hence $\Lambda_{h}(\Sigma)$ is built as in (5.10) on the resulting even number of elements covering $\Sigma$.

5.4. The main results. As a consequence of the results and analyses in Sections 5.1, 5.2, and 5.3, we can establish the following theorems.

Theorem 5.3. Let $\mathbf{H}_{h}\left(\Omega_{\mathrm{S}}\right), \mathbf{H}_{h}\left(\Omega_{\mathrm{D}}\right), L_{h}\left(\Omega_{\mathrm{S}}\right)$, and $L_{h}\left(\Omega_{\mathrm{D}}\right)$ be the Raviart-Thomas finite element subspaces given in (5.1) and define

$$
\begin{aligned}
\mathbb{H}_{h}\left(\Omega_{\mathrm{S}}\right) & :=\left\{\boldsymbol{\tau}: \Omega_{\mathrm{S}} \rightarrow \mathbb{R}^{2 \times 2}: \quad \mathbf{c}^{t} \boldsymbol{\tau} \in \mathbf{H}_{h}\left(\Omega_{\mathrm{S}}\right) \quad \forall \mathbf{c} \in \mathbb{R}^{2}\right\}, \\
\mathbb{H}_{h, 0}\left(\Omega_{\mathrm{S}}\right) & :=\mathbb{H}_{h}\left(\Omega_{\mathrm{S}}\right) \cap \mathbb{H}_{0}\left(\mathbf{d i v} ; \Omega_{\mathrm{S}}\right), \\
\mathbf{L}_{h}\left(\Omega_{\mathrm{S}}\right) & :=L_{h}\left(\Omega_{\mathrm{S}}\right) \times L_{h}\left(\Omega_{\mathrm{S}}\right), \\
L_{h, 0}\left(\Omega_{\mathrm{D}}\right) & :=L_{h}\left(\Omega_{\mathrm{D}}\right) \cap L_{0}^{2}\left(\Omega_{\mathrm{D}}\right) .
\end{aligned}
$$

Assume that $\mathcal{T}_{h}^{\mathrm{S}}$ and $\mathcal{T}_{h}^{\mathrm{D}}$ are quasiuniform in a neighborhood of $\Sigma$ and that $\Lambda_{h}(\Sigma)$ (and hence $\Lambda_{h}(\Sigma):=\Lambda_{h}(\Sigma) \times \Lambda_{h}(\Sigma)$ ) is given by any of the three options described above. Then the Galerkin scheme (4.7) with the discrete spaces $\mathbb{X}_{h, 0}:=\mathbb{H}_{h, 0}\left(\Omega_{\mathrm{S}}\right) \times$ $\mathbf{H}_{h}\left(\Omega_{\mathrm{D}}\right) \times \boldsymbol{\Lambda}_{h}(\Sigma) \times \Lambda_{h}(\Sigma)$ and $\mathbb{M}_{h, 0}:=\mathbf{L}_{h}\left(\Omega_{\mathrm{S}}\right) \times L_{h, 0}\left(\Omega_{\mathrm{D}}\right) \times \mathbb{R}$, has a unique solution $\left(\underline{\boldsymbol{\sigma}}_{h}, \underline{\mathbf{u}}_{h}\right) \in \mathbb{X}_{h, 0} \times \mathbb{M}_{h, 0}$, which satisfies the corresponding stability and Cea estimates.

Proof. It follows by gathering the results from Sections 4 and 5 .

Theorem 5.4. Assume the same hypotheses of Theorem 5.3. Then the Galerkin scheme (4.19) with the spaces $\mathbb{X}_{h}:=\mathbb{H}_{h}\left(\Omega_{\mathrm{S}}\right) \times \mathbf{H}_{h}\left(\Omega_{\mathrm{D}}\right) \times \boldsymbol{\Lambda}_{h}(\Sigma) \times \Lambda_{h}(\Sigma)$ and $\mathbb{M}_{h}:=\mathbf{L}_{h}\left(\Omega_{\mathrm{S}}\right) \times L_{h, 0}\left(\Omega_{\mathrm{D}}\right)$, has a unique solution $\left(\underline{\boldsymbol{\sigma}}_{h}, \underline{\mathbf{u}}_{h}\right) \in \mathbb{X}_{h} \times \mathbb{M}_{h}$, which satisfies the corresponding stability and Cea estimates.

Proof. It follows from Theorem 5.3 and the equivalence between (4.7) and (4.19).

In order to provide the rate of convergence of the Galerkin scheme (4.7), we now recall the approximation properties of the subspaces involved (see, e.g. [4], 8], [25]):

(AP1) For $\star \in\{\mathrm{S}, \mathrm{D}\}$, for each $\delta \in(0,1]$, and for each $\tau \in \mathbf{H}^{\delta}\left(\Omega_{\star}\right)$ with $\operatorname{div} \tau \in$ $H^{\delta}\left(\Omega_{\star}\right)$, there exists $\tau_{h} \in \mathbf{H}_{h}\left(\Omega_{\star}\right)$ such that

$$
\left\|\tau-\tau_{h}\right\|_{\operatorname{div}, \Omega_{\star}} \leq C h^{\delta}\left\{\|\tau\|_{\delta, \Omega_{\star}}+\|\operatorname{div} \tau\|_{\delta, \Omega_{\star}}\right\} .
$$

(AP2) For $\star \in\{\mathrm{S}, \mathrm{D}\}$, for each $\delta \in[0,1]$, and for each $q \in L^{2}\left(\Omega_{\star}\right)$, there exists $q_{h} \in L_{h}\left(\Omega_{\star}\right)$ such that

$$
\left\|q-q_{h}\right\|_{0, \Omega_{\star}} \leq C h^{\delta}\|q\|_{\delta, \Omega_{\star}} .
$$


(AP3) For each $\delta \in[0,1]$ and for each $\xi \in H^{1 / 2+\delta}(\Sigma)$, there exists $\xi_{h} \in \Lambda_{h}(\Sigma)$ such that

$$
\left\|\xi-\xi_{h}\right\|_{1 / 2, \Sigma} \leq C h^{\delta}\|\xi\|_{1 / 2+\delta, \Sigma} .
$$

The following theorem provides the theoretical rate of convergence of the Galerkin scheme (4.7) (equivalently (4.19)), under suitable regularity assumptions on the exact solution.

Theorem 5.5. Let $(\underline{\boldsymbol{\sigma}}, \underline{\mathbf{u}}) \in \mathbb{X}_{0} \times \mathbb{M}_{0}$ and $\left(\underline{\boldsymbol{\sigma}}_{h}, \underline{\mathbf{u}}_{h}\right) \in \mathbb{X}_{h, 0} \times \mathbb{M}_{h, 0}$ be the unique solutions of the continuous and discrete formulations (3.2) and (4.7), respectively. Assume that there exists $\delta \in(0,1]$ such that $\boldsymbol{\sigma}_{\mathrm{S}} \in \mathbb{H}^{\delta}\left(\Omega_{\mathrm{S}}\right)$, $\operatorname{div} \boldsymbol{\sigma}_{\mathrm{S}} \in \mathbf{H}^{\delta}\left(\Omega_{\mathrm{S}}\right)$, $\mathbf{u}_{\mathrm{D}} \in \mathbf{H}^{\delta}\left(\Omega_{\mathrm{D}}\right)$, and $\operatorname{div} \mathbf{u}_{\mathrm{D}} \in H^{\delta}\left(\Omega_{\mathrm{D}}\right)$. Then, $\mathbf{u}_{\mathrm{S}} \in \mathbf{H}^{1+\delta}\left(\Omega_{\mathrm{S}}\right), p_{\mathrm{D}} \in H^{1+\delta}\left(\Omega_{\mathrm{D}}\right)$, $\varphi \in \mathbf{H}^{1 / 2+\delta}(\Sigma), \lambda \in H^{1 / 2+\delta}(\Sigma)$, and there exists $C>0$, independent of $h$ and the continuous and discrete solutions, such that

$$
\begin{aligned}
& \left\|(\underline{\boldsymbol{\sigma}}, \underline{\mathbf{u}})-\left(\underline{\boldsymbol{\sigma}}_{h}, \underline{\mathbf{u}}_{h}\right)\right\|_{\mathbb{X} \times \mathbb{M}} \leq C h^{\delta}\left\{\left\|\boldsymbol{\sigma}_{\mathrm{S}}\right\|_{\delta, \Omega_{\mathrm{S}}}+\left\|\operatorname{div} \boldsymbol{\sigma}_{\mathrm{S}}\right\|_{\delta, \Omega_{\mathrm{S}}}\right. \\
& \left.+\left\|\mathbf{u}_{\mathrm{D}}\right\|_{\delta, \Omega_{\mathrm{D}}}+\left\|\operatorname{div} \mathbf{u}_{\mathrm{D}}\right\|_{\delta, \Omega_{\mathrm{D}}}+\left\|\mathbf{u}_{\mathrm{S}}\right\|_{1+\delta, \Omega_{\mathrm{S}}}+\left\|p_{\mathrm{D}}\right\|_{1+\delta, \Omega_{\mathrm{D}}}\right\} .
\end{aligned}
$$

Proof. We first recall from Theorem 2.1 that $\nabla \mathbf{u}_{\mathrm{S}}=\nu^{-1} \boldsymbol{\sigma}_{\mathrm{S}}^{d}$ and $\nabla p_{\mathrm{D}}=-\mathbf{K}^{-1} \mathbf{u}_{\mathrm{D}}$, which implies that $\mathbf{u}_{\mathrm{S}} \in \mathbf{H}^{1+\delta}\left(\Omega_{\mathrm{S}}\right)$ and $p_{\mathrm{D}} \in H^{1+\delta}\left(\Omega_{\mathrm{D}}\right)$, whence $\boldsymbol{\varphi}=-\left.\mathbf{u}_{\mathrm{S}}\right|_{\Sigma} \in$ $\mathbf{H}^{1 / 2+\delta}(\Sigma)$ and $\lambda=\left.p_{\mathrm{D}}\right|_{\Sigma} \in H^{1 / 2+\delta}(\Sigma)$. The rest of the proof follows from the corresponding Cea estimate, the above approximation properties, and the fact that, thanks to the trace theorem in $\Omega_{\mathrm{S}}$ and $\Omega_{\mathrm{D}}$, respectively, there holds

$$
\|\varphi\|_{1 / 2+\delta, \Sigma} \leq c\left\|\mathbf{u}_{\mathrm{S}}\right\|_{1+\delta, \Omega_{\mathrm{S}}} \text { and }\|\lambda\|_{1 / 2+\delta, \Sigma} \leq c\left\|p_{\mathrm{D}}\right\|_{1+\delta, \Omega_{\mathrm{D}}} .
$$

We end this section by commenting that one should be able to extend the analysis of Section 5, without difficulties, to the case of Raviart-Thomas finite element subspaces of higher order. In this case, given $k \geq 1, \mathrm{RT}_{0}(T)$ is replaced by $\mathrm{RT}_{k}(T):=\left[\mathbb{P}_{k}(T)\right]^{2} \oplus \mathbb{P}_{k}(T)\left(\begin{array}{l}x_{1} \\ x_{2}\end{array}\right)$, and $\Lambda_{h}(\Sigma)$ is defined in terms of piecewise polynomials of degre $2 k+1$.

\section{Numerical Results}

In this section we present three examples illustrating the performance of the Galerkin scheme (4.19) (equivalently (4.7)) with the subspaces $\mathbb{X}_{h}:=\mathbb{H}_{h}\left(\Omega_{\mathrm{S}}\right) \times$ $\mathbf{H}_{h}\left(\Omega_{\mathrm{D}}\right) \times \boldsymbol{\Lambda}_{h}(\Sigma) \times \Lambda_{h}(\Sigma)$ and $\mathbb{M}_{h}:=\mathbf{L}_{h}\left(\Omega_{\mathrm{S}}\right) \times L_{h, 0}\left(\Omega_{\mathrm{D}}\right)$ defined in Section 5 , In particular, we adopt the third option from Section 5.3 to choose the space $\Lambda_{h}(\Sigma)$ of continuous piecewise linear functions on $\Sigma$.

We now introduce additional notation. The variable $N$ stands for the number of degrees of freedom defining $\mathbb{X}_{h}$ and $\mathbb{M}_{h}$, and the individual errors are denoted by

$$
\begin{aligned}
& \mathrm{e}\left(\boldsymbol{\sigma}_{\mathrm{S}}\right):=\left\|\boldsymbol{\sigma}_{\mathrm{S}}-\boldsymbol{\sigma}_{\mathrm{S}, h}\right\|_{\operatorname{div}, \Omega_{\mathrm{S}}}, \quad \mathrm{e}\left(\mathbf{u}_{\mathrm{S}}\right):=\left\|\mathbf{u}_{\mathrm{S}}-\mathbf{u}_{\mathrm{S}, h}\right\|_{\text {div }, \Omega_{\mathrm{S}}}, \\
& \mathrm{e}\left(\mathbf{u}_{\mathrm{D}}\right):=\left\|\mathbf{u}_{\mathrm{D}}-\mathbf{u}_{\mathrm{D}, h}\right\|_{\mathrm{div}, \Omega_{\mathrm{D}}}, \quad \mathrm{e}\left(p_{\mathrm{D}}\right):=\left\|p_{\mathrm{D}}-p_{\mathrm{D}, h}\right\|_{0, \Omega_{\mathrm{D}}}, \\
& \mathrm{e}(\boldsymbol{\varphi}):=\left\|\boldsymbol{\varphi}-\boldsymbol{\varphi}_{h}\right\|_{1 / 2, \Sigma}, \quad \mathrm{e}(\lambda):=\left\|\lambda-\lambda_{h}\right\|_{1 / 2, \Sigma},
\end{aligned}
$$

where $\underline{\boldsymbol{\sigma}}_{h}:=\left(\boldsymbol{\sigma}_{\mathrm{S}, h}, \mathbf{u}_{\mathrm{D}, h}, \varphi_{h}, \lambda_{h}\right) \in \mathbb{X}_{h}$ and $\underline{\mathbf{u}}_{h}:=\left(\mathbf{u}_{\mathrm{S}, h}, p_{\mathrm{D}, h}\right) \in \mathbb{M}_{h}$ constitute the unique solution of (4.19).

Also, we let $r\left(\boldsymbol{\sigma}_{\mathrm{S}}\right), r\left(\mathbf{u}_{\mathrm{S}}\right), r\left(\mathbf{u}_{\mathrm{D}}\right), r\left(p_{\mathrm{D}}\right), r(\boldsymbol{\varphi})$, and $r(\lambda)$ be the experimental rates of convergence given by

$$
r(\%):=\frac{\log \left(\mathrm{e}(\%) / \mathrm{e}^{\prime}(\%)\right)}{\log \left(h / h^{\prime}\right)} \quad \text { for each } \quad \% \in\left\{\boldsymbol{\sigma}_{\mathrm{S}}, \mathbf{u}_{\mathrm{S}}, \mathbf{u}_{\mathrm{D}}, p_{\mathrm{D}}, \boldsymbol{\varphi}, \lambda\right\},
$$


where $h$ and $h^{\prime}$ denote two consecutive meshsizes with errors e and $\mathrm{e}^{\prime}$.

In what follows we describe the data of the examples. In all cases we choose for simplicity $\nu=1, \mathbf{K}=\mathrm{I}$, the identity matrix of $\mathbb{R}^{2 \times 2}$, and $\kappa=1$.

In Example 1 we consider the regions $\left.\Omega_{\mathrm{D}}:=\right]-1 / 2,1 / 2[\times]-1 / 2,1 / 2[$ and $\left.\Omega_{\mathrm{S}}:=\right]-1,1[\times]-1,1\left[\backslash \Omega_{\mathrm{D}}\right.$, which represents a porous medium completely surrounded by a fluid. Then we choose the data $\mathbf{f}_{\mathrm{S}}$ and $f_{\mathrm{D}}$ so that the exact solution is given by

$$
\begin{gathered}
\mathbf{u}_{\mathrm{S}}\left(x_{1}, x_{2}\right)=\left(\begin{array}{c}
-4\left(x_{1}^{2}-1\right)^{2}\left(x_{2}^{2}-1\right) x_{2} \\
4\left(x_{1}^{2}-1\right)\left(x_{2}^{2}-1\right)^{2} x_{1}
\end{array}\right) \text { in } \Omega_{\mathrm{S}}, \\
p_{\mathrm{S}}\left(x_{1}, x_{2}\right)=-\sin \left(x_{1}\right) e^{x_{2}} \text { in } \Omega_{\mathrm{S}},
\end{gathered}
$$

and

$$
p_{\mathrm{D}}\left(x_{1}, x_{2}\right)=-\sin \left(x_{1}\right) e^{x_{2}} \quad \text { in } \quad \Omega_{\mathrm{D}} .
$$

In Example 2 we let $\Omega_{\mathrm{S}}$ and $\Omega_{\mathrm{D}}$ be the polygonal domains delimited by the set of points $\{(-1,0),(1,0),(1,1),(-1 / 2,1)\}$ and $\{(-1 / 2,-1),(1 / 2,-1),(1,0),(-1,0)\}$, respectively, which constitutes a particular case of a fluid over a porous medium, and choose the data $\mathbf{f}_{\mathrm{S}}$ and $f_{\mathrm{D}}$ so that the exact solution is given by

$$
\mathbf{u}_{\mathrm{S}}\left(x_{1}, x_{2}\right)=\left(\begin{array}{c}
2\left(x_{2}-1\right)\left(x_{1}-1\right)^{2}\left(2 x_{1}-x_{2}+2\right)\left(2 x_{1}-2 x_{2}+3\right) \\
-2\left(x_{2}-1\right)^{2}\left(x_{1}-1\right)\left(4 x_{1}-x_{2}\right)\left(2 x_{1}-x_{2}+2\right)
\end{array}\right) \text { in } \Omega_{\mathrm{S}},
$$

and

$$
p_{\mathrm{D}}\left(x_{1}, x_{2}\right)=\sin \left(x_{1}\right)\left(4 x_{1}^{2}-\left(x_{2}+2\right)^{2}\right)^{2}\left(x_{2}+1\right)^{2} \quad \text { in } \quad \Omega_{\mathrm{D}} .
$$

Finally, in Example 3 we consider the domains $\left.\Omega_{\mathrm{S}}:=\right]-1,1[\times] 0,1[$ and $\left.\Omega_{\mathrm{D}}:=\right]-1,1[\times]-1,0[$, which constitutes another case of a fluid over a porous medium, and take the data $\mathbf{f}_{\mathrm{S}}$ and $f_{\mathrm{D}}$ given by

$$
\mathbf{f}_{\mathrm{S}}\left(x_{1}, x_{2}\right)=\left(\begin{array}{c}
-4 \sin \left(x_{1} x_{2}\right) x_{1}+\exp \left(x_{2}^{3}\right) \\
4 \exp \left(3 x_{1}\right)+4 x_{2}
\end{array}\right)
$$

and

$$
f_{\mathrm{D}}\left(x_{1}, x_{2}\right)=x_{1}^{3}\left(\exp \left(x_{2}^{2}\right)-0.5\right)
$$

This example corresponds to a more realistic situation in which the exact solution is unknown.

The numerical results shown below were obtained using a MATLAB implementation. In Tables 2 and 3 we present the convergence history of Examples 1 and 2 , respectively, for a set of shape-regular triangulations of the computational domain $\bar{\Omega}_{\mathrm{S}} \cup \bar{\Omega}_{\mathrm{D}}$. We see there that the dominant error in both examples is given by $\mathrm{e}\left(\boldsymbol{\sigma}_{\mathrm{S}}\right)$, though this is more evident in Example 1 . In addition, we observe that the rate of convergence $O(h)$ provided by Theorem 5.5 for $\delta=1$ is attained by all the unknowns. Next, in Figures 4 and 5 (resp. Figures 6] and 7) we display the approximate and exact values of some components of the solution of Example 1 for $N=144641$ (resp. Example 2 for $N=273071$ ). It is clear from these figures that the finite element subspaces employed provide very accurate approximations to the unknowns in both domains. In particular, the quality of these approximations is not affected at all by the strong oscillations of some solutions. The shape-regular character of the meshes is illustrated in Figure 8 for Example 2. 
Next, in Table 4 we present the convergence history of Example 3 for a set of shape-regular triangulations of the computational domain $\bar{\Omega}_{\mathrm{S}} \cup \bar{\Omega}_{\mathrm{D}}$. The errors and experimental rates of convergence shown there are computed by considering the discrete solution obtained with a finer mesh $(N=984068)$ as the exact solution. Similarly, as for Examples 1 and 2, we observe that the rate of convergence $O(h)$ is attained by all the unknowns, and the dominant error is also given by $\mathrm{e}\left(\boldsymbol{\sigma}_{\mathrm{S}}\right)$. Next, in Figures 9, 10, and 11] we show some components of the approximate solutions obtained for $N=123396$. Note that in this example the normal on the interface $\Sigma:=(-1,1) \times\{0\}$ is given by $\mathbf{n}=(0,-1)^{t}$, and hence the first transmission condition becomes the equality of the second components of $\mathbf{u}_{\mathrm{S}}$ and $\mathbf{u}_{\mathrm{D}}$. This can be verified at the discrete level in Figure 10 where we display 3D and 2D joint pictures of the second components of $\mathbf{u}_{\mathrm{S}, h}$ and $\mathbf{u}_{\mathrm{D}, h}$.

Summarizing, the numerical results reported here confirm the good performance of the mixed finite element scheme (4.19) with Raviart-Thomas finite element subspaces of lowest order in $\Omega_{\mathrm{S}}$ and $\Omega_{\mathrm{D}}$, and continuous piecewise linear functions on the interface $\Sigma$, for different geometries of the coupled problem.

We end this paper by mentioning that the only reason for restricting here to $2 \mathrm{D}$ is the simple fact that in our previous works [20] and [21] we assumed that dimension. We believe, however, that the present results should be extended, with minor modifications, to the three-dimensional case. Indeed, it is easy to see that the sections concerning the model problem and the general analysis of the continuous and discrete formulations, should look more or less the same as the ones provided here. Eventual technical difficulties, not too hard to solve, nevertheless, might appear only in the analogue of Section 5 , probably in the construction of the discrete liftings and the verification of the discrete inf-sup condition (5.9). We hope to address this issue in a separate work.

TABle 2. Degrees of freedom, meshsizes, errors, and rates of convergence (EXAMPLE 1).

\begin{tabular}{c|c||c|c||c|c||c|c}
$N$ & $h$ & $\mathrm{e}\left(\boldsymbol{\sigma}_{\mathrm{S}}\right)$ & $r\left(\boldsymbol{\sigma}_{\mathrm{S}}\right)$ & $\mathrm{e}\left(\mathbf{u}_{\mathrm{S}}\right)$ & $r\left(\mathbf{u}_{\mathrm{S}}\right)$ & $\mathrm{e}\left(\mathbf{u}_{\mathrm{D}}\right)$ & $r\left(\mathbf{u}_{\mathrm{D}}\right)$ \\
\hline 641 & 0.3536 & 5.2974 & & 0.3622 & & 0.1204 & \\
2401 & 0.1768 & 2.6875 & 1.0277 & 0.1802 & 1.0573 & 0.0584 & 1.0957 \\
9281 & 0.0884 & 1.3468 & 1.0219 & 0.0900 & 1.0269 & 0.0289 & 1.0406 \\
36481 & 0.0442 & 0.6737 & 1.0121 & 0.0450 & 1.0128 & 0.0144 & 1.0178 \\
144641 & 0.0221 & 0.3369 & 1.0062 & 0.0225 & 1.0064 & 0.0072 & 1.0064 \\
$N$ & $h$ & $\mathrm{e}\left(p_{\mathrm{D}}\right)$ & $r\left(p_{\mathrm{D}}\right)$ & $\mathrm{e}(\boldsymbol{\varphi})$ & $r(\boldsymbol{\varphi})$ & $\mathrm{e}(\lambda)$ & $r(\lambda)$ \\
\hline 641 & 0.3536 & 0.0645 & & 1.0988 & & 0.2572 & \\
2401 & 0.1768 & 0.0320 & 1.0615 & 0.5390 & 1.0787 & 0.1260 & 1.0807 \\
9281 & 0.0884 & 0.0160 & 1.0253 & 0.2661 & 1.0441 & 0.0619 & 1.0514 \\
36481 & 0.0442 & 0.0080 & 1.0128 & 0.1321 & 1.0232 & 0.0306 & 1.0294 \\
144641 & 0.0221 & 0.0040 & 1.0064 & 0.0658 & 1.0119 & 0.0152 & 1.0159
\end{tabular}



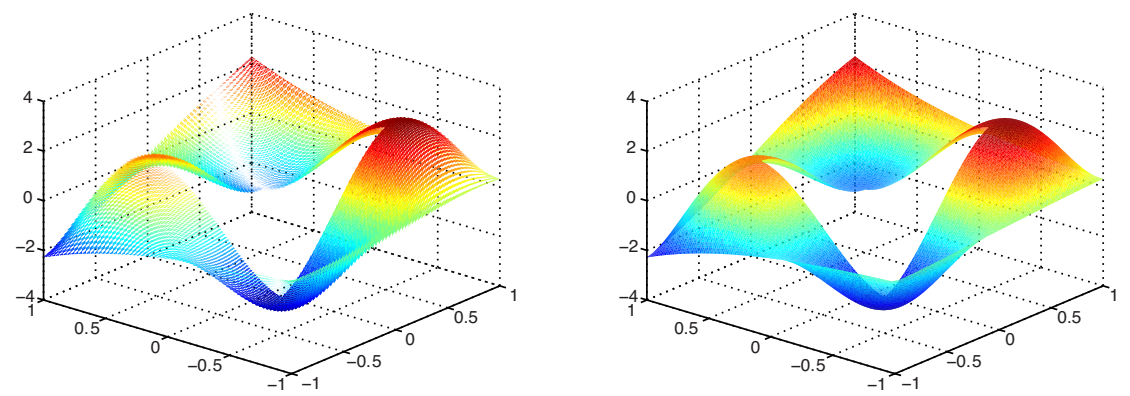

Figure 4. Components $(1,1)$ of $\boldsymbol{\sigma}_{\mathrm{S}, h}$ and $\boldsymbol{\sigma}_{\mathrm{S}}$ (Example 1)
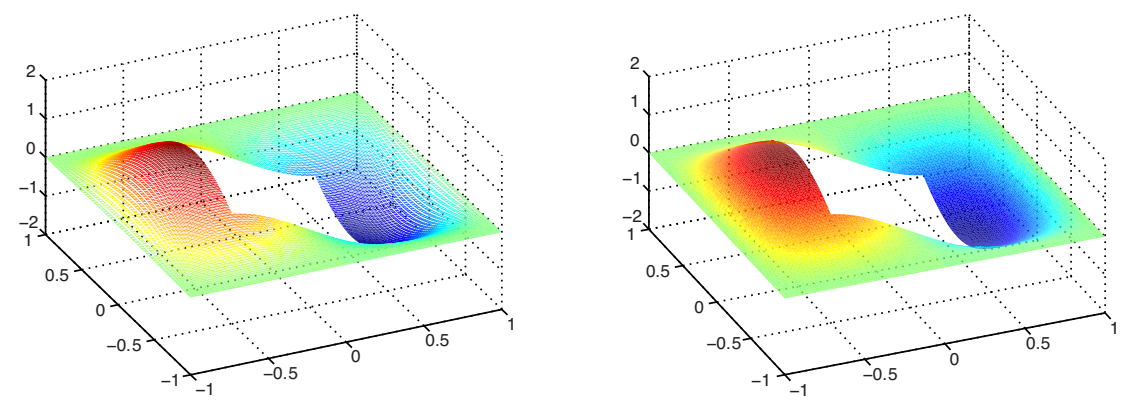

FiguRE 5. Second components of $\mathbf{u}_{\mathrm{S}, h}$ and $\mathbf{u}_{\mathrm{S}}$ (EXAmple 1)

TABle 3. Degrees of freedom, meshsizes, errors, and rates of convergence (EXAMPLE 2).

\begin{tabular}{c|c||c|c||c|c||c|c}
$N$ & $h$ & $\mathrm{e}\left(\boldsymbol{\sigma}_{\mathrm{S}}\right)$ & $r\left(\boldsymbol{\sigma}_{\mathrm{S}}\right)$ & $\mathrm{e}\left(\mathbf{u}_{\mathrm{S}}\right)$ & $r\left(\mathbf{u}_{\mathrm{S}}\right)$ & $\mathrm{e}\left(\mathbf{u}_{\mathrm{D}}\right)$ & $r\left(\mathbf{u}_{\mathrm{D}}\right)$ \\
\hline 219 & 0.2500 & 15.4093 & & 1.5670 & & 11.2401 & \\
1225 & 0.0885 & 6.6580 & 0.9748 & 0.7182 & 0.9063 & 5.1261 & 0.9121 \\
7368 & 0.0347 & 2.4934 & 1.0948 & 0.2789 & 1.0543 & 1.6395 & 1.2707 \\
44595 & 0.0117 & 0.9809 & 1.0363 & 0.1135 & 0.9989 & 0.6796 & 0.9783 \\
273071 & 0.0035 & 0.4041 & 0.9789 & 0.0461 & 0.9931 & 0.2742 & 1.0017 \\
$N$ & $h$ & $\mathrm{e}\left(p_{\mathrm{D}}\right)$ & $r\left(p_{\mathrm{D}}\right)$ & $\mathrm{e}(\boldsymbol{\varphi})$ & $r(\boldsymbol{\varphi})$ & $\mathrm{e}(\lambda)$ & $r(\lambda)$ \\
\hline 219 & 0.2500 & 1.0956 & & 11.6289 & & 6.2759 & \\
1225 & 0.0885 & 1.0870 & 0.0091 & 4.0529 & 1.2245 & 4.2773 & 0.4454 \\
7368 & 0.0347 & 0.1317 & 2.3529 & 1.2658 & 1.2972 & 1.1155 & 1.4982 \\
44595 & 0.0117 & 0.0434 & 1.2324 & 0.5188 & 0.9907 & 0.3315 & 1.3478 \\
273071 & 0.0035 & 0.0180 & 0.9709 & 0.2010 & 1.0468 & 0.1286 & 1.0452
\end{tabular}



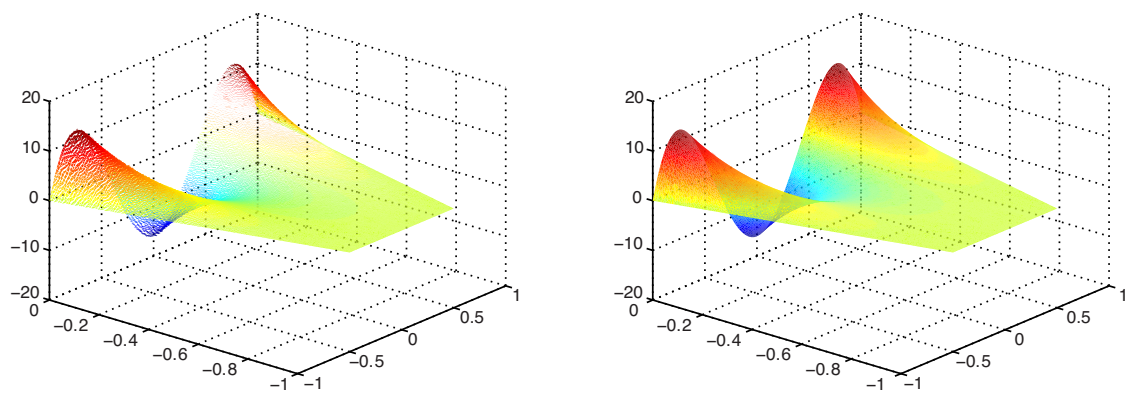

Figure 6. First components of $\mathbf{u}_{\mathrm{D}, h}$ and $\mathbf{u}_{\mathrm{D}}$ (EXAmple 2)
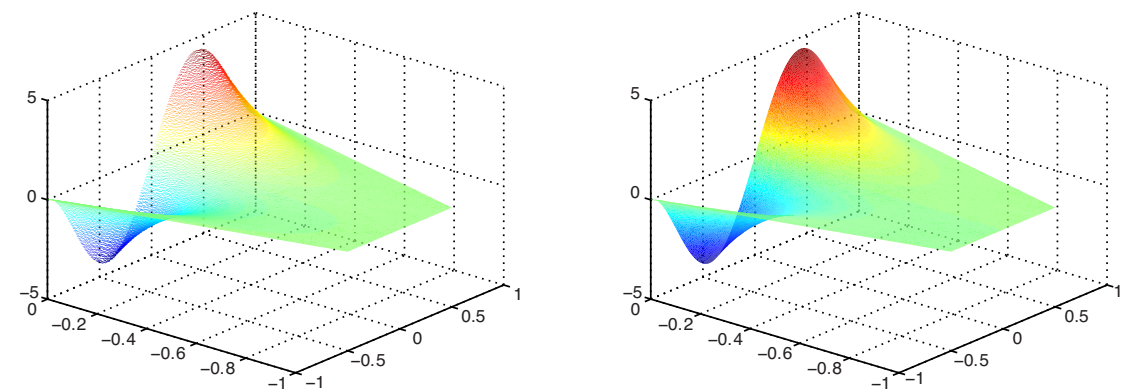

FiguRe 7. $p_{\mathrm{D}, h}$ and $p_{\mathrm{D}}$ (EXAMPle 2$)$
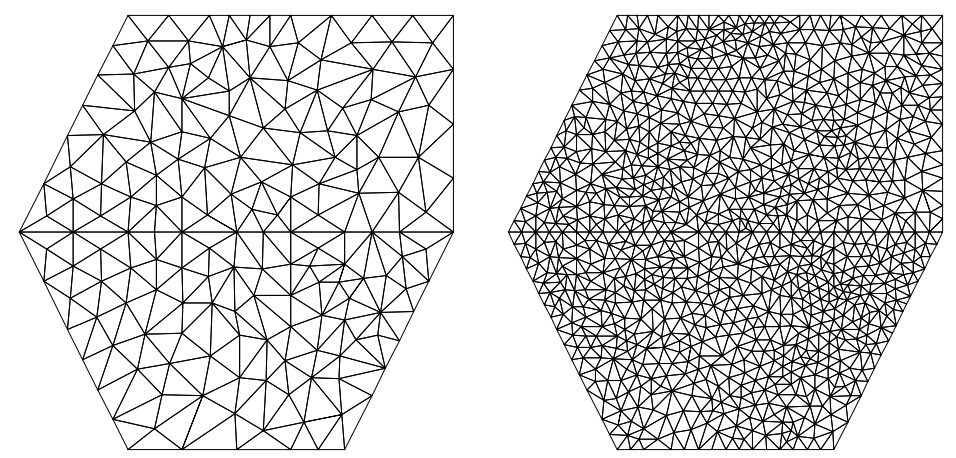

Figure 8. Meshes for $N=1225$ and $N=7368$ (Example 2) 
TABle 4. Degrees of freedom, meshsizes, errors, and rates of convergence (EXAMPLE 3).

\begin{tabular}{c|c||c|c||c|c||c|c}
$N$ & $h$ & $\mathrm{e}\left(\boldsymbol{\sigma}_{\mathrm{S}}\right)$ & $r\left(\boldsymbol{\sigma}_{\mathrm{S}}\right)$ & $\mathrm{e}\left(\mathbf{u}_{\mathrm{S}}\right)$ & $r\left(\mathbf{u}_{\mathrm{S}}\right)$ & $\mathrm{e}\left(\mathbf{u}_{\mathrm{D}}\right)$ & $r\left(\mathbf{u}_{\mathrm{D}}\right)$ \\
\hline 516 & 0.3536 & 6.7467 & & 0.1873 & & 0.1911 & \\
1988 & 0.1768 & 3.4022 & 1.0152 & 0.0758 & 1.3414 & 0.0921 & 1.0823 \\
7812 & 0.0884 & 1.6984 & 1.0153 & 0.0340 & 1.1717 & 0.0456 & 1.0273 \\
30980 & 0.0442 & 0.8482 & 1.0080 & 0.0164 & 1.0584 & 0.0227 & 1.0126 \\
123396 & 0.0221 & 0.4243 & 1.0024 & 0.0081 & 1.0208 & 0.0114 & 0.9967 \\
$N$ & $h$ & $\mathrm{e}\left(p_{\mathrm{D}}\right)$ & $r\left(p_{\mathrm{D}}\right)$ & $\mathrm{e}(\boldsymbol{\varphi})$ & $r(\boldsymbol{\varphi})$ & $\mathrm{e}(\lambda)$ & $r(\lambda)$ \\
\hline 516 & 0.3536 & 0.0301 & \multicolumn{1}{|c||}{} & 0.5915 & & 0.1324 & \\
1988 & 0.1768 & 0.0112 & 1.4659 & 0.2838 & 1.0890 & 0.0498 & 1.4499 \\
7812 & 0.0884 & 0.0050 & 1.1786 & 0.1449 & 0.9824 & 0.0185 & 1.4472 \\
30980 & 0.0442 & 0.0024 & 1.0655 & 0.0761 & 0.9349 & 0.0062 & 1.5870 \\
123396 & 0.0221 & 0.0012 & 1.0031 & 0.0401 & 0.9271 & 0.0019 & 1.7115
\end{tabular}
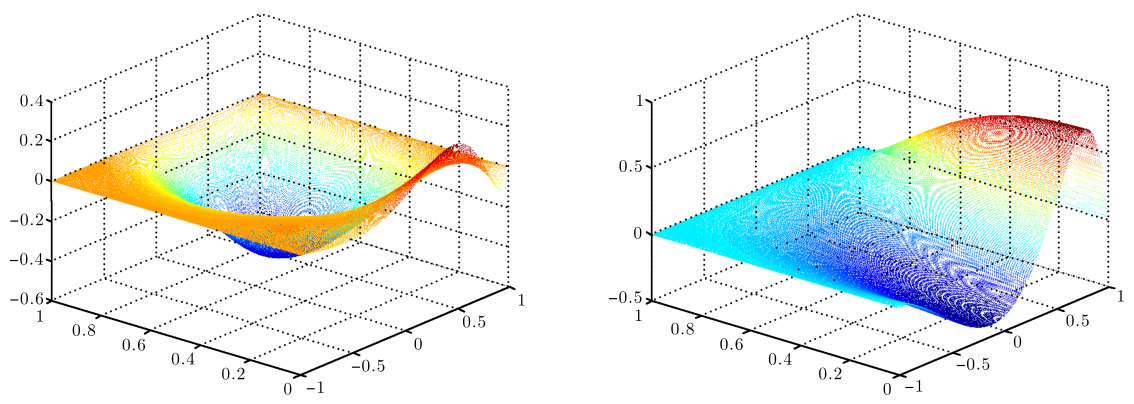

Figure 9. First and second components of $\mathbf{u}_{\mathrm{S}, h}$ (EXAmple 3)
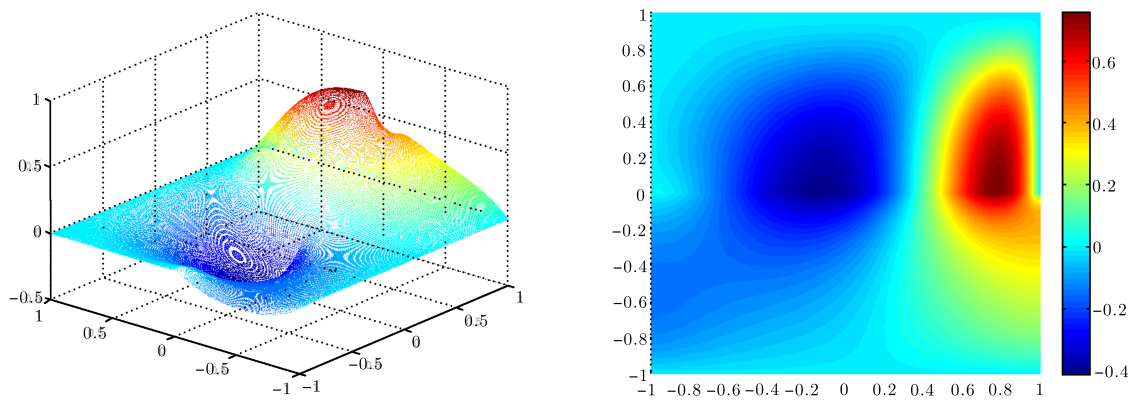

Figure 10. Second components of $\mathbf{u}_{\mathrm{S}, h}$ and $\mathbf{u}_{\mathrm{D}, h}$ (ExAmple 3 ) 

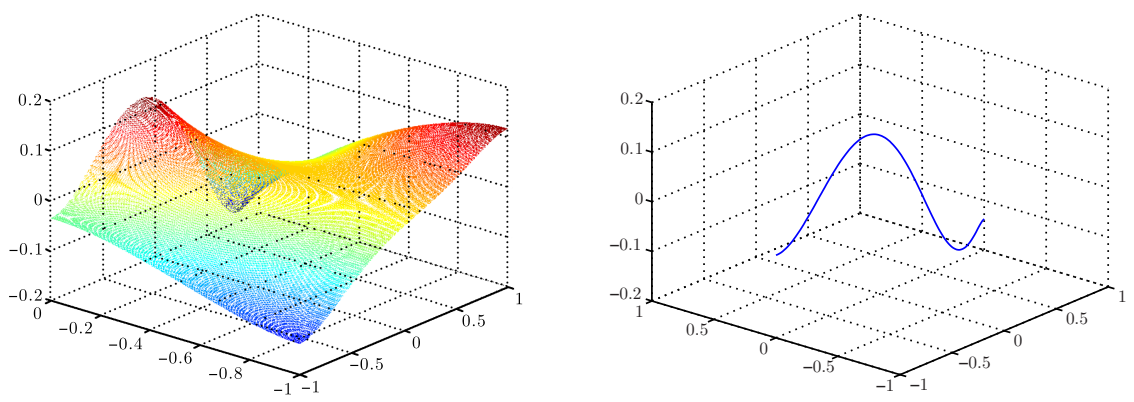

Figure 11. $p_{\mathrm{D}, h}$ and $\lambda_{h}$ (EXAmple 3$)$

\section{ACKNOWLEDGMENTS}

The authors thank Professor Luc Tartar (Carnegie Mellon University) for his help in clarifying some concepts on interpolation inequalities in the Hilbert frame, which served us to locate and mend a fallacious argument in the first version of Lemma 5.2

\section{REFERENCES}

1. A. Agouzal and J.-M. Thomas, An extension theorem for equilibrium finite elements spaces. Japan Journal of Industrial and Applied Mathematics, vol. 13, 2, pp. 257-266, (1996). MR.1394627 (97g:65220)

2. T. ARBogast And D.S. Brunson, A computational method for approximating a Darcy-Stokes system governing a vuggy porous medium. Computational Geosciences, vol. 11, 3, pp. 207-218, (2007). MR2344200 (2009b:76155)

3. D.N. Arnold, J. Douglas and Ch.P. Gupta, A family of higher order mixed finite element methods for plane elasticity. Numerische Mathematik, vol. 45, 1, pp. 1-22, (1984). MR.761879 (86a:65112)

4. I. BABUŠKA AND A.K. AzIZ, Survey lectures on the mathematical foundations of the finite element method. In: The Mathematical Foundations of the Finite Element Method with Applications to Partial Differential Equations, A.K. Aziz (editor), Academic Press, New York, (1972). MR0421106 (54:9111)

5. I. BABUŠKA AND G.N. GATICA, On the mixed finite element method with Lagrange multipliers. Numerical Methods for Partial Differential Equations, vol. 19, 2, pp. 192-210, (2003). MR.1958060 (2004b:65174)

6. G. Beavers AND D. Joseph, Boundary conditions at a naturally impermeable wall. Journal of Fluid Mechanics, vol. 30, pp. 197-207, (1967).

7. C. Bernardi, F. Hecht, and O. Pironneau, Coupling Darcy and Stokes equations for porous media with cracks. ESAIM: Mathematical Modelling and Numerical Analysis, vol. 39, 1, pp. 7-35, (2005). MR.2136198 (2006a:76107)

8. F. Brezzi And M. Fortin, Mixed and Hybrid Finite Element Methods. Springer Series in Computational Mathematics, 15. Springer-Verlag, New York, 1991. MR.1115205 (92d:65187)

9. E. Burman And P. Hansbo, Stabilized Crouzeix-Raviart elements for the Darcy-Stokes problem. Numerical Methods for Partial Differential Equations, vol. 21, 5, pp. 986-997, (2005). MR.2154230 (2006i:65190)

10. E. Burman And P. Hansbo, A unified stabilized method for Stokes' and Darcy's equations. Journal of Computational and Applied Mathematics, vol. 198, 1, pp. 35-51, (2007). MR 2250387 (2007i:65076)

11. M.R. Correa, Stabilized Finite Element Methods for Darcy and Coupled Stokes-Darcy Flows. D.Sc. Thesis, LNCC, Petrópolis, Rio de Janeiro, Brasil (in Portuguese), (2006). 
12. M.R. Correa AND A.F.D. Loula, A unified mixed formulation naturally coupling Stokes and Darcy flows. Computer Methods in Applied Mechanics and Engineering, vol. 198, 33-36, pp. 2710-2722, (2009).

13. M. Discacciati, Domain Decomposition Methods for the Coupling of Surface and Groundwater Flows. Ph.D. Thesis, Ecole Polytechnique Fédérale de Lausanne, 2004.

14. M. Discacciati, E. Miglio, and A. Quarteroni, Mathematical and numerical models for coupling surface and groundwater flows. Applied Numerical Mathematics, vol. 43, pp. 57-74, (2002). MR $1936102(2003 \mathrm{~h}: 76087)$

15. V. Domínguez And F.-J. SAyas, Stability of discrete liftings. Comptes Rendus Mathématique. Académie des Sciences. Paris, vol. 337, 12, pp. 805-808, (2003). MR 2033124

16. V.J. Ervin, E.W. Jenkins, And S. Sun, Coupled generalized nonlinear Stokes flow with flow through a porous medium. SIAM Journal on Numerical Analysis, vol. 47, 2, pp. 929-952, (2009). MR 2485439 (2010b:65254)

17. A. Friedman, Foundations of Modern Analysis. Holt, Rinehart and Winston, Inc., New YorkMontreal, Que.-London, 1970. MR0275100 (43:858)

18. J. Galvis AND M. SARKIS, Non-matching mortar discretization analysis for the coupling Stokes-Darcy equations. Electronic Transactions on Numerical Analysis, vol. 26, pp. 350-384, (2007). MR2391227 (2009a:76120)

19. G.N. Gatica, N. Heuer, And S. Meddahi, On the numerical analysis of nonlinear twofold saddle point problems. IMA Journal of Numerical Analysis, vol. 23, 2, pp. 301-330, (2003). MR 1975268 (2004b:65183)

20. G.N. Gatica, S. Meddahi, and R. Oyarzúa, A conforming mixed finite-element method for the coupling of fluid flow with porous media flow. IMA Journal of Numerical Analysis, vol. 29, 1, pp. 86-108, (2009). MR2470941 (2010b:76118)

21. G.N. Gatica, R. OYARZúA, AND F.-J. SAYAS, Convergence of a family of Galerkin discretizations for the Stokes-Darcy coupled problem. Numerical Methods for Partial Differential Equations DOI 10.1002/num.

22. G.N. Gatica And F.-J. SaYas, Characterizing the inf-sup condition on product spaces. Numerische Mathematik, vol. 109, 2, pp. 209-231, (2008). MR2385652 (2009g:47034)

23. V. Girault and P.-A. Raviart, Finite Element Approximation of the Navier-Stokes Equations. Lecture Notes in Mathematics, 749. Springer-Verlag, Berlin-New York, 1979. MR548867 (83b:65122)

24. P. Grisvard, Singularities in Boundary Value Problems. Recherches en Mathématiques Appliquées, 22. Masson, Paris; Springer-Verlag, Berlin, 1992. MR,1173209 (93h:35004)

25. R. Hiptmair, Finite elements in computational electromagnetism. Acta Numerica, vol. 11, pp. 237-339, (2002). MR2009375 (2004k:78028)

26. W. JäGER AND M. Mikelic, On the interface boundary condition of Beavers, Joseph, and Saffman. SIAM Journal on Applied Mathematics, vol. 60, pp. 1111-1127, (2000). MR.1760028 (2001e:76122)

27. T. Karper, K.-A. Mardal, and R. Winther, Unified finite element discretizations of coupled Darcy-Stokes flow. Numerical Methods for Partial Differential Equations, vol. 25, 2, pp. 311-326, (2009). MR2483769 (2010a:65240)

28. W.J. LAYTON, F. SChiEWECK, AND I. Yotov, Coupling fluid flow with porous media flow. SIAM Journal on Numerical Analysis, vol. 40, 6, pp. 2195-2218, (2003). MR.1974181 (2004c:76048)

29. A. MASUd, A stabilized mixed finite element method for Darcy-Stokes flow. International Journal for Numerical Methods in Fluids, vol. 54, 6-8, pp. 665-681, (2008). MR2333004 (2008b:76120)

30. W. MCLEAN, Strongly Elliptic Systems and Boundary Integral Equations. Cambridge University Press, 2000. MR.1742312 (2001a:35051)

31. A. Quarteroni and A. Valli, Numerical Approximation of Partial Differential Equations. Springer Series in Computational Mathematics, 23. Springer-Verlag, Berlin, Heidelberg, 1994. MR.1299729 (95i:65005)

32. M.L. RAPÚN AND F.-J. SAYAS, Boundary integral approximation of a heat-diffusion problem in time-harmonic regime. Numerical Algorithms, vol. 41, 2, pp. 127-160, (2006). MR.2222969 (2007c:65114) 
33. B. Riviere, Analysis of a discontinuous finite element method for coupled Stokes and Darcy problems. Journal of Scientific Computing, vol. 22-23, pp. 479-500, (2005). MR 2142206 (2006b:65175)

34. B. Riviere AND I. Yotov, Locally conservative coupling of Stokes and Darcy flows. SIAM Journal on Numerical Analysis, vol. 42, 5, pp. 1959-1977, (2005). MR2139232 (2006a:76035)

35. H. Rui And R. Zhang, A unified stabilized mixed finite element method for coupling Stokes and Darcy flows. Computer Methods in Applied Mechanics and Engineering, vol. 198, 33-36, pp. 2692-2699, (2009). MR2532369 (2010g:76045)

36. P. Saffman, On the boundary condition at the surface of a porous media. Studies in Applied Mathematics, vol. 50, pp. 93-101, (1971).

37. J.M. Urquiza, D. N'Dri, A. Garon, and M.C. Delfour, Coupling Stokes and Darcy equations. Applied Numerical Mathematics, vol. 58, 5, pp. 525-538, (2008). MR2407730 (2009a:76053)

38. X. XIE, J. XU, and G. Xue, Uniformly stable finite element methods for Darcy-StokesBrinkman models. Journal of Computational Mathematics, vol. 26, 3, pp. 437-455, (2008). MR.2421892 (2009g:76087)

Ci²MA and Departamento de Ingeniería Matemática, Universidad de Concepción, Casilla 160-C, Concepción, Chile

E-mail address: ggatica@ing-mat.udec.cl

Departamento de Ingeniería Matemática, Universidad de Concepción, Casilla 160-C, CONCEPCión, Chile

E-mail address: royarzua@ing-mat.udec.cl

Department of Mathematical Sciences, University of Delaware, Newark, Delaware 19716, USA

E-mail address: fjsayas@math.udel.edu 